\title{
Cyclopentadienone Synthesis by Rhodium(I)-Catalyzed [3 + 2] Cycloaddition Reactions of Cyclopropenones and Alkynes
}

\author{
Paul A. Wender,* Thomas J. Paxton, and Travis J. Williams
}

I. General Procedures.

II. Preparative and Experimental Details for New Compounds.

III. Selected ${ }^{1}$ H NMR Spectra for New Compounds.

IV. X-Ray Crystallographic Data for Compound 3.

\section{General Procedures.}

General methods: air and moisture sensitive reactions were carried out in oven-dried glassware sealed with rubber septa under a positive pressure of dry nitrogen from a manifold unless otherwise indicated. Sensitive liquids and solutions were transferred via syringe or stainless steel cannula. Reactions were run using Teflon ${ }^{\mathrm{TM}}$-coated magnetic stir bars. Elevated temperatures were maintained in temperature regulated oil baths. Unless otherwise indicated, cycloadditions were run in borosilicate test tubes. Organic solutions were concentrated using a Buchi or Heildoph rotary evaporator. Thin layer chromatography plates were visualized (as needed) by ultraviolet light and treatment with acidic $p$ anisaldehyde stain or potassium permanganate stain followed by gentle heating. Chromatographic purification of products was accomplished by flash chromatography. Silica gel 60, 230-400 mesh was purchased from EM Science. 1,2-dichloroethane was freshly distilled over calcium hydride. Tetrahydrofuran, toluene, and dichloromethane were purified with a Solvtek ${ }^{\mathrm{TM}}$ solvent purification system using alumina columns. Where indicated, tetrahydrofuran was distilled over benzophenone ketyl (benzophenone plus sodium metal). Other solvents were used directly from commercial suppliers, unless otherwise specified. $\left[\mathrm{RhCl}(\mathrm{CO})_{2}\right]_{2}$ was purchased from Strem Chemicals. Diphenylcyclopropenone (1), alkynes, and other materials were purchased from Aldrich Chemical Co. and used without further purification unless otherwise noted. Copper(I) iodide was purified prior to use by the method of Kauffman. ${ }^{1}$

NMR spectra were measured on a Varian INOVA $500\left({ }^{1} \mathrm{H}\right.$ at $500 \mathrm{MHz},{ }^{13} \mathrm{C}$ at $\left.125 \mathrm{MHz}\right)$, Varian Mercury $400\left({ }^{1} \mathrm{H}\right.$ at $400 \mathrm{MHz},{ }^{13} \mathrm{C}$ at $\left.100 \mathrm{MHz}\right)$, Varian Gemini $200\left({ }^{1} \mathrm{H}\right.$ at $200 \mathrm{MHz},{ }^{13} \mathrm{C}$ at $\left.50 \mathrm{MHz}\right) \mathrm{magnetic}$ resonance spectrometer. Data for ${ }^{1} \mathrm{H}$ NMR spectra are reported as follows: chemical shift $(\delta, \mathrm{ppm})$, multiplicity $(\mathrm{s}=$ singlet, $\mathrm{d}=$ doublet, $\mathrm{t}=$ triplet, $\mathrm{q}=$ quartet, $\mathrm{dd}=$ doublet of doublets, $\mathrm{dt}=$ doublet of triplets, $\mathrm{qd}=$ quartet of doublets, $\mathrm{ddd}=$ doublet of doublet of doublets, $\mathrm{m}=$ multiplet), coupling constant $(\mathrm{Hz})$, and integration. Data for ${ }^{13} \mathrm{C}$ are reported in terms of chemical shift and quantity of carbons. Infrared spectra were recorded on a Perkin-Elmer Spectrum BX or ThermoNicolet IR300 Fourier transform spectrometer (FTIR) and are reported as follows: wavenumbers $\left(\mathrm{cm}^{-1}\right)$, description $(\mathrm{w}=$ weak, $\mathrm{m}=$ medium, $\mathrm{s}=$ strong, $\mathrm{b}=$ broad). High-resolution mass spectra (HRMS) were recorded at the NIH regional mass spectrometry facility at the University of California, San Francisco. Reported mass values are with error limits of \pm 4 millimass unit. MALDI data was recorded on an Voyager Biosystems MALDI apparatus. Melting points were measured with a Thomas-Hoover Unimelt ${ }^{\mathrm{TM}}$ apparatus. Elemental analyses $(\% \mathrm{C}, \% \mathrm{H}, \% \mathrm{~N}, \% \mathrm{~F})$ were determined by Desert Analytics laboratory of Tucson, Arizona. X-ray data collection was accomplished by CHEXRAY (University of California, Berkeley). X-ray data was solved at Stanford by Xavier Ottenwaelder, Prateek Verma, and T. D. P. Stack. 
II. Preparative and Experimental Details for New Compounds.<smiles>O=c1c([PH2+])c1-c1ccccc1</smiles>

1

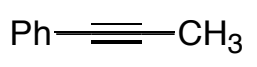

2

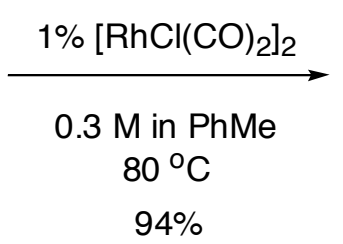<smiles>CC1=C(c2ccccc2)C(=O)C(c2ccccc2)=C1c1ccccc1</smiles>

3

Cyclopentadienone 3:

Diphenylcyclopropenone (1, $375 \mu \mathrm{mol}, 77.3 \mathrm{mg})$ and 1-phenyl-1-propyne (2, $250 \mu \mathrm{mol}, 25.1 \mathrm{mg}, 27 \mu \mathrm{L})$ were weighed out into a test tube with stir bar under nitrogen. To this was added toluene (Solvtek ${ }^{\mathrm{TM}}, 750$ $\mu \mathrm{L})$ and $\left[\mathrm{RhCl}(\mathrm{CO})_{2}\right]_{2}(1.0 \mathrm{mg}, 2.5 \mu \mathrm{mol})$. The tube was capped with a septum and heated at $80{ }^{\circ} \mathrm{C}$ for 2 hours under nitrogen. The resulting solution was cooled to room temperature and then purified by flash column chromatography eluting with $100 \mathrm{~mL}$ petroleum ether followed by $150 \mathrm{~mL} 5 \%$ diethyl ether/petroleum ether. Product-containing fractions were concentrated under reduced pressure to leave cyclopentadienone $\mathbf{3}$ as a deep purple solid (76 mg, 94\%).

Large-Scale: Diphenylcyclopropenone (1, $12 \mathrm{mmol}, 2.47 \mathrm{~g})$ and $\left[\mathrm{RhCl}(\mathrm{CO})_{2}\right]_{2}(46.7 \mathrm{mg}, 0.12 \mathrm{mmol}, 1.5$ mol\%) were weighed out into a round-bottom flask with stir bar under nitrogen and capped with a septum. To this was added toluene (Solvtek ${ }^{\mathrm{TM}}, 27 \mathrm{~mL}$ ) and 1-phenyl-1-propyne (2, $8 \mathrm{mmol}, 929 \mathrm{mg}, 1.00$ $\mathrm{mL}$ ). The reaction was heated at $80{ }^{\circ} \mathrm{C}$ for 6.5 hours under nitrogen. At this point, TLC indicated complete consumption of 2 . The reaction was cooled to room temperature and then concentrated under reduced pressure. The concentrate was purified by flash column chromatography eluting with $500 \mathrm{~mL}$ pentane followed by $500 \mathrm{~mL} \mathrm{5 \%} \mathrm{ethyl} \mathrm{acetate/pentane.} \mathrm{Product-containing} \mathrm{fractions} \mathrm{were} \mathrm{concentrated}$ under reduced pressure to leave cyclopentadienone 3 as a deep purple solid (2.19 g, 85\%).

$\mathbf{R}_{\mathbf{f}}: 0.57$ in 19:1, pentane: ether (appears red in visible light).

m. p. $145-148{ }^{\circ} \mathrm{C}$.

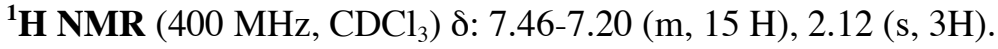

${ }^{13} \mathrm{C}$ NMR $\left(\mathrm{CDCl}_{3}, 100 \mathrm{MHz}\right) \delta: 200.7,154.7,154.4,134.0,131.6,130.9,130.1$ (2C), 129.8 (2C), 128.9 (2C), 128.9 (2C), 128.8, 128.5 (2C), 128.2 (2C), 127.6, 127.6, 125.9, 124.9, 14.9.

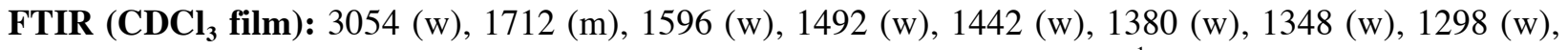
1113 (w), 1073 (w), 1002 (w), $989(w), 910(w), 796(w), 738(\mathrm{~m}), 695(\mathrm{~s}) \mathrm{cm}^{-1}$.

HRMS for $\mathrm{C}_{24} \mathrm{H}_{18} \mathrm{O}$ calc'd: $322.1358 \mathrm{~g} / \mathrm{mol}$, found: $322.1356 \mathrm{~g} / \mathrm{mol}$. 


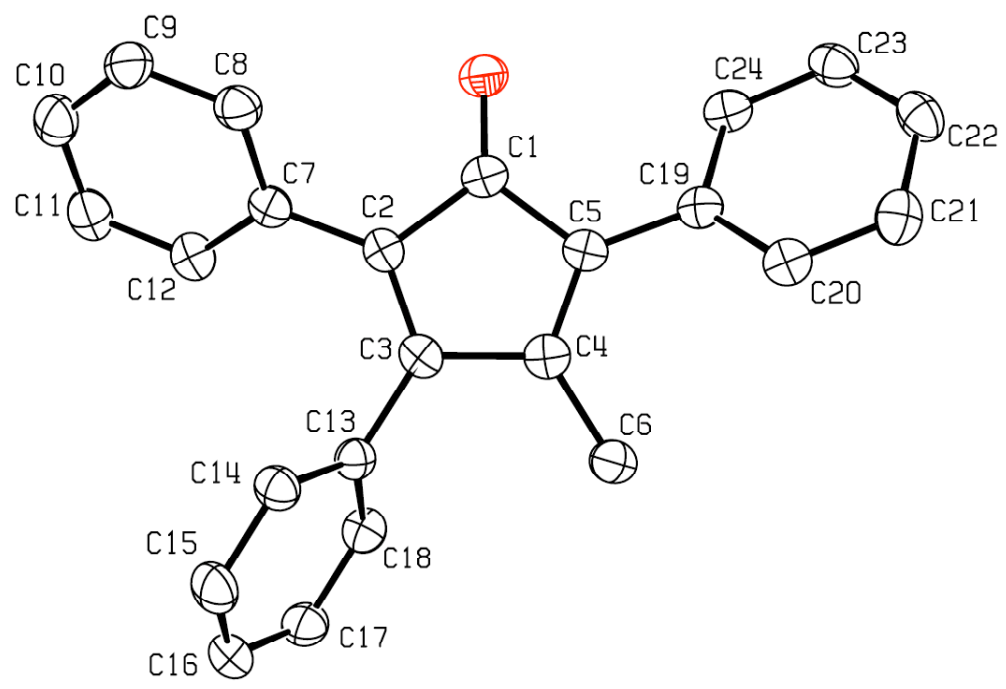

ORTEP diagram of 3. Ellipsoids drawn at the $50 \%$ probability level.
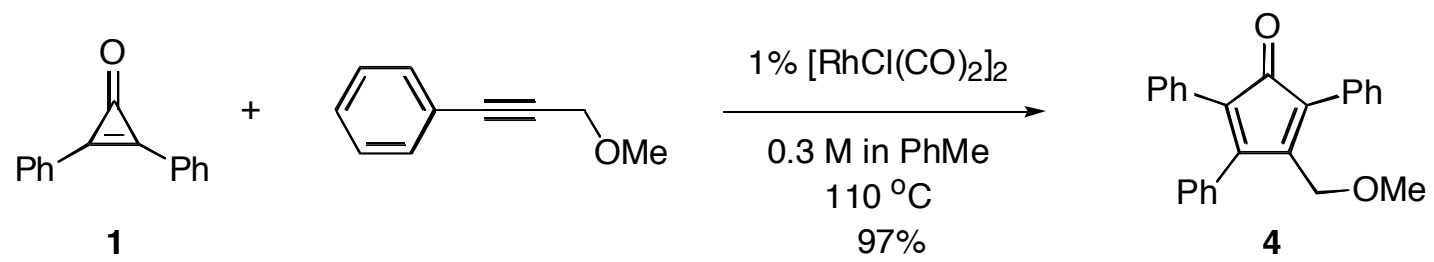

Cyclopentadienone 4:

Phenylpropargyl methyl ether ${ }^{2}$ (147 mg, $1 \mathrm{mmol}$ ), diphenylcyclopropenone (1, $1.51 \mathrm{mmol}, 310 \mathrm{mg}$ ), and $\left[\mathrm{RhCl}(\mathrm{CO})_{2}\right]_{2}(10.0 \mu \mathrm{mol}, 3.9 \mathrm{mg})$ were weighed out into a test tube with stir bar under nitrogen. To this was added toluene (Solvtek ${ }^{\mathrm{TM}}, 3.3 \mathrm{~mL}$ ). The tube was capped with a septum and heated in an oil bath for 4 hours at $110{ }^{\circ} \mathrm{C}$ under nitrogen. The resulting solution was then purified by flash column chromatography eluting with $300 \mathrm{~mL}$ petroleum ether followed by $500 \mathrm{~mL} \mathrm{5 \%}$ diethyl ether/petroleum ether. Product-containing fractions were concentrated under reduced pressure to leave cyclopentadienone 4 as a purple solid (340 mg, 97\%).

$\mathbf{R}_{\mathbf{f}}: 0.41$ in 19:1, petroleum ether: ether (appears red in visible light).

m. p. $114-118^{\circ} \mathrm{C}$.

${ }^{1} \mathbf{H}$ NMR $\left(500 \mathrm{MHz}, \mathrm{CDCl}_{3}\right)$ \&: 7.56-7.54 (m, 2H), 7.47-7.37 (m, 8H), 7.22-7.20 (m, 5H), $4.11(\mathrm{~s}, 2 \mathrm{H})$, $3.28(\mathrm{~s}, 3 \mathrm{H})$.

${ }^{13} \mathrm{C}$ NMR $\left(\mathrm{CDCl}_{3}, 125 \mathrm{MHz}\right) \delta: 200.6,155.6,150.1,133.6,130.7,130.5,129.9$ (2C), 129.8 (2C), 129.5, $128.8,128.6(2 \mathrm{C}), 128.6(2 \mathrm{C}), 128.4(2 \mathrm{C}), 128.3,128.0(2 \mathrm{C}), 127.4,124.8,65.5,58.4$.

FTIR (CDCl ${ }_{3}$ film): 3054 (w), 2917 (m), 2848 (w), 1712 (s), 1493 (w), 1443 (m), 1392 (w), 1347 (w), 1302 (w), 1189 (w), 1156 (w), 1091 (s), 1028 (m), 942 (w), 797 (w), 739 (w), 695 (s) $\mathrm{cm}^{-1}$.

HRMS for $\mathrm{C}_{25} \mathrm{H}_{20} \mathrm{O}_{2}$ calc'd: $352.1463 \mathrm{~g} / \mathrm{mol}$, found: $352.1461 \mathrm{~g} / \mathrm{mol}$.

Elemental Analysis for $\mathrm{C}_{25} \mathrm{H}_{20} \mathrm{O}_{2}$ calc'd: $85.20 \%$ C, $5.72 \% \mathrm{H}, 9.08 \%$ O; found $84.80 \%$ C, $5.84 \% \mathrm{H}$. 


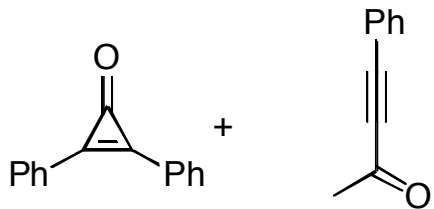

1

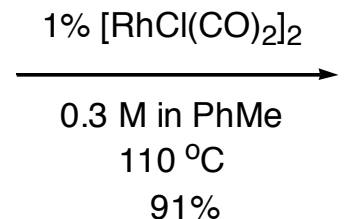

$91 \%$

Cyclopentadienone 5:

Diphenylcyclopropenone (1, $77.3 \mathrm{mg}, 375 \mu \mathrm{mol})$ and 4-phenyl-but-3-yn-2-one (36 $\mathrm{mg}, 250 \mu \mathrm{mol})$ were weighed out into a test tube with stir bar under nitrogen. To this was added toluene $\left(750 \mu \mathrm{L}\right.$, Solvtek $\left.{ }^{\mathrm{TM}}\right)$ and $\left[\mathrm{RhCl}(\mathrm{CO})_{2}\right]_{2}(1.0 \mathrm{mg}, 2.5 \mu \mathrm{mol})$. The tube was then capped with a septum and then heated at $110{ }^{\circ} \mathrm{C}$ for 18 hours under nitrogen. The crude reaction mixture was then cooled to room temperature and then purified by flash column chromatography eluting with diethyl ether and pentane (1:19). Productcontaining fractions were collected and concentrated under reduced pressure to give a purple solid $\mathbf{5}$ (80 $\mathrm{mg}, 91 \%$ yield).

m. p. $132-134{ }^{\circ} \mathrm{C}$.

$\mathbf{R}_{\mathbf{f}}: 0.33$ in 24:1, pentane: ethyl acetate (appears red in visible light).

${ }^{1} \mathbf{H}$ NMR $\left(\mathrm{CDCl}_{3}, 500 \mathrm{MHz}\right)$ ઈ: 7.49-7.45 (m, 2H), 7.45-7.32 (m, 6H), 7.27-7.19 (m, 7H), 2.13 (s, 3H).

${ }^{13} \mathrm{C}$ NMR $\left(\mathrm{CDCl}_{3}, 125 \mathrm{MHz}\right) \delta: 201.7,199.6,153.6,152.2,132.8,130.0,129.9$ (2C), 129.5 (2C), 129.3, 129.2, 129.1, 128.7 (2C), 128.5 (2C), 128.1 (2C), 128.0 (2C), 127.8, 126.5, 124.9, 31.4.

FTIR (thin film): 3727 (w), 3054 (w), 2360 (s), 2341 (s), 1717 (s), 1699 (s), 1694 (m), 1490 (w), 1443 (m), $1352(\mathrm{~m}), 1181(\mathrm{w}), 1112(\mathrm{~m}), 694(\mathrm{~s}), 668(\mathrm{~m}) \mathrm{cm}^{-1}$.

HRMS for $\mathrm{C}_{25} \mathrm{H}_{18} \mathrm{O}_{2}$ calc'd: $350.1307 \mathrm{~g} / \mathrm{mol}$, found $350.1312 \mathrm{~g} / \mathrm{mol}$.

Elemental Analysis for $\mathrm{C}_{25} \mathrm{H}_{18} \mathrm{O}_{2}$ calc'd: $85.69 \%$ C; $5.19 \% \mathrm{H} ; 9.13 \% \mathrm{O}$; found $85.62 \% \mathrm{C} ; 5.37 \% \mathrm{H}$.
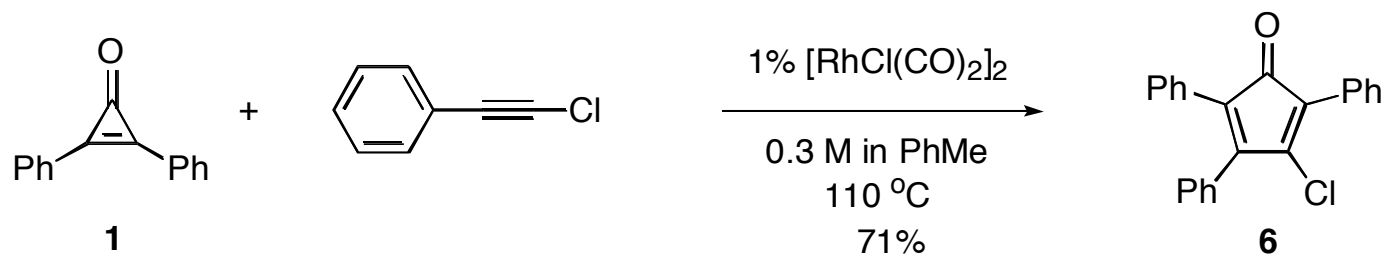

Cyclopentadienone 6:

Chloroethynyl benzene ${ }^{3}(20.0 \mathrm{mg}, 146 \mu \mathrm{mol})$, diphenylcyclopropenone ( $\left.\mathbf{1}, 45.2 \mathrm{mg}, 220 \quad \mu \mathrm{mol}\right)$, and $\left[\mathrm{RhCl}(\mathrm{CO})_{2}\right]_{2}(1.5 \mu \mathrm{mol}, 0.6 \mathrm{mg})$ were weighed out into a test tube with stir bar under nitrogen. To this was added toluene (Solvtek ${ }^{\mathrm{TM}}, 440 \mu \mathrm{L}$ ). The tube was capped with a septum and heated in an oil bath for 6 hours at $110{ }^{\circ} \mathrm{C}$ under nitrogen. The resulting solution was purified by flash column chromatography eluting with $1 \%$ diethyl ether/pentane. Product fractions were collected and concentrated under reduced pressure to give cyclopentadienone 6 as a red solid (35.4 mg, $71 \%$ ).

m. p. $147-150{ }^{\circ} \mathrm{C}$.

${ }^{1} \mathbf{H}$ NMR $\left(400 \mathrm{MHz}, \mathrm{CDCl}_{3}\right.$ ) $\delta: 7.74$ (apparent dd, $\left.J_{1}=8.5 \mathrm{~Hz}, J_{2}=1.3 \mathrm{~Hz}, 2 \mathrm{H}\right), 7.49-7.40$ (m, 8H), 7.25 (apparent s, 5H). 
${ }^{13} \mathrm{C}$ NMR $\left(\mathrm{CDCl}_{3}, 100 \mathrm{MHz}\right) \delta: 196.7,149.8,149.5,131.2,130.1$ (2C), 129.6, 129.5 (2C), 129.4 (2C), $129.3,129.2,128.4(2 \mathrm{C}), 128.4,128.3$ (2C), $128.2(2 \mathrm{C}), 128.0,125.3,123.8$.

FTIR $\left(\mathrm{CDCl}_{3}\right.$ film): 3055 (w), 1717 (s), 1598 (w), 1490 (m), 1443 (m), 1346 (m), 1256 (m), 1156 (w), 1115 (s), 1073 (w), 1027 (m), 906 (w), 792 (m), $738(\mathrm{~m}), 713(\mathrm{~s}) \mathrm{cm}^{-1}$.

HRMS for $\mathrm{C}_{23} \mathrm{H}_{15} \mathrm{Cl}$ calc'd: $342.0811 \mathrm{~g} / \mathrm{mol}$, found: $342.0805 \mathrm{~g} / \mathrm{mol}$.

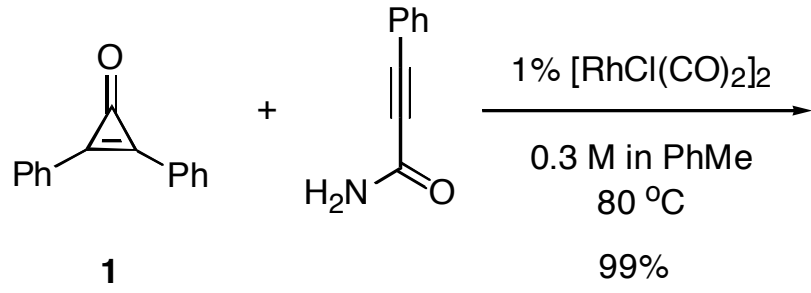

Cyclopentadienone 7:

Diphenylcyclopropenone (1, $77.3 \mathrm{mg}, 375 \mu \mathrm{mol})$ and 3-phenyl-propynoic acid amide (36.3 $\mathrm{mg}, 250$ $\mu \mathrm{mol})$ were weighed out into a test tube with stir bar under nitrogen. To this was added toluene (1.250 $\mathrm{mL}$, Solvtek $\left.{ }^{\mathrm{TM}}\right)$ and $\left[\mathrm{RhCl}(\mathrm{CO})_{2}\right]_{2}(1.0 \mathrm{mg}, 2.5 \mu \mathrm{mol})$. The tube was capped with a septum and the reaction was heated at $80{ }^{\circ} \mathrm{C}$ for 12 hours under nitrogen. The crude reaction mixture was then cooled to room temperature and then purified by flash column chromatography eluting with methanol and chloroform (1:99). Product-containing fractions were collected and concentrated under reduced pressure to give cyclopentadienone 7 as a purple solid ( $87 \mathrm{mg}, 99 \%$ yield).

m. p. $202-204{ }^{\circ} \mathrm{C}$.

$\mathbf{R}_{\mathbf{f}}: 0.68$ in 6:5, ethyl acetate: pentane (appears purple in visible light).

${ }^{1} \mathbf{H}$ NMR $\left(\mathrm{CDCl}_{3}, 500 \mathrm{MHz}\right)$ ઈ: 7.63-7.60 (m, 2H), 7.46-7.31 (m, 8H), 7.27-7.23 (m, 3H), 7.21-7.18 (m, $2 \mathrm{H}), 5.73(\mathrm{~s}, 1 \mathrm{H}), 5.60(\mathrm{~s}, 1 \mathrm{H})$.

${ }^{13} \mathrm{C}$ NMR $\left(\mathrm{CDCl}_{3}, 125 \mathrm{MHz}\right) \delta: 199.5,166.9,160.4,153.0,147.0,132.5,130.1,130.0$ (2C), 129.5 (2C), 129.3 (2C), 129.1 (2C), 128.6 (3C), 128.2 (3C), 127.9, 126.4, 124.8.

FTIR (thin film): 3402 (m), 3339 (w), 3081 (w), 3057 (w), 1716 (s), 1661 (s), 1597 (m), 1493 (w), 1443 (m), 1344 (w), 1344 (w), $1261(\mathrm{w}), 1130$ (w), 1114 (w), 909 (w), 796 (w), 735 (m), 715 (m), 694 (s) cm . $^{-1}$

HRMS for $\mathrm{C}_{24} \mathrm{H}_{17} \mathrm{NO}_{2}$ calc'd: $351.1259 \mathrm{~g} / \mathrm{mol}$, found $351.1249 \mathrm{~g} / \mathrm{mol}$.

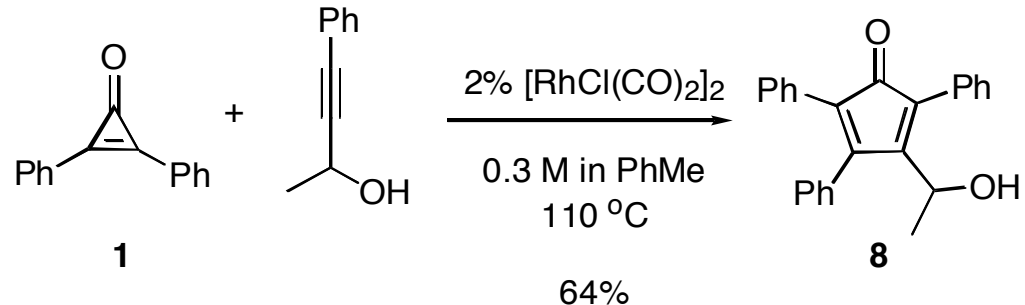

Alcohol 8:

Diphenylcyclopropenone (1, $186 \mathrm{mg}, 903 \mu \mathrm{mol})$ and 4-phenyl-but-3-yn-2-ol (88 mg, $602 \mu \mathrm{mol})$ were weighed out into a test tube with stir bar under nitrogen. To this was added toluene $\left(2.0 \mathrm{~mL}\right.$, Solvtek $\left.{ }^{\mathrm{TM}}\right)$ and $\left[\mathrm{RhCl}(\mathrm{CO})_{2}\right]_{2}(2.4 \mathrm{mg}, 6.0 \mu \mathrm{mol})$. The tube was then capped with a septum and then heated at $110{ }^{\circ} \mathrm{C}$ for 12 hours under nitrogen. TLC indicated incomplete conversion. To this was added more 
$\left[\mathrm{RhCl}(\mathrm{CO})_{2}\right]_{2}(2.4 \mathrm{mg}, 6.0 \mu \mathrm{mol})$ and the reaction was heated at $110{ }^{\circ} \mathrm{C}$ for an additional 11 hours under nitrogen. The crude reaction mixture was then cooled to room temperature and then purified by flash column chromatography eluting with using ethyl acetate and pentane (1:6). Product-containing fractions were collected and concentrated under reduced pressure to give a red solid $\mathbf{8}$ (135 mg, 64\% yield).

m. p. $59-61{ }^{\circ} \mathrm{C}$.

$\mathbf{R}_{\mathbf{f}}: 0.30$ in 6:1, pentane: ethyl acetate (appears red spot on plate).

${ }^{1} \mathbf{H}$ NMR $\left(\mathrm{CDCl}_{3}, 400 \mathrm{MHz}\right) \delta: 7.46-7.34(\mathrm{~m}, 10 \mathrm{H}), 7.20-7.13(\mathrm{~m}, 5 \mathrm{H}), 4.93\left(\mathrm{dq}, J_{\text {quart }}=6.6 \mathrm{~Hz}, J_{\text {double }}=\right.$ $6.5 \mathrm{~Hz}, 1 \mathrm{H}), 1.60(\mathrm{~d}, J=6.4 \mathrm{~Hz}, 1 \mathrm{H}), 1.26(\mathrm{~d}, J=6.7 \mathrm{~Hz}, 3 \mathrm{H})$.

${ }^{13} \mathrm{C}$ NMR $\left(\mathrm{CDCl}_{3}, 100 \mathrm{MHz}\right) \delta: 200.6,157.4,154.6,134.7,130.7,130.4,130.1$ (2C), 129.8 (2C), 128.8 (2C), 128.7 (2C), 128.6, 128.3 (2C), 128.1, 127.9 (2C), 127.4, 126.4, 125.5, 65.3, 22.7.

FTIR (thin film): 3523 (b, m), 3054 (w), 2929 (w) 2360 (m), 2341 (m), 1710 (s), 1596 (w), 1491 (m), 1443 (m), 1347 (w), 1263 (w), 1158 (w), 1090 (m), 1072 (m), 1029 (m), 1015 (m), 910 (m), 796 (m), 740 (m), $697(\mathrm{~s}) \mathrm{cm}^{-1}$.

HRMS for $\mathrm{C}_{25} \mathrm{H}_{20} \mathrm{O}_{2}$ calc'd: $352.1463 \mathrm{~g} / \mathrm{mol}$, found $352.1460 \mathrm{~g} / \mathrm{mol}$.

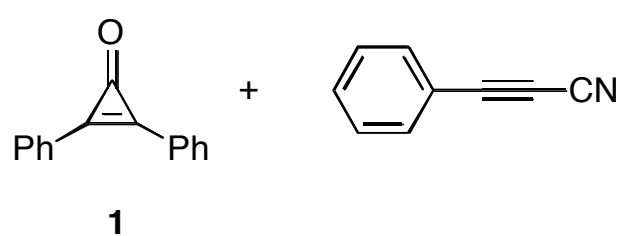

Cyclopentadienone 9:
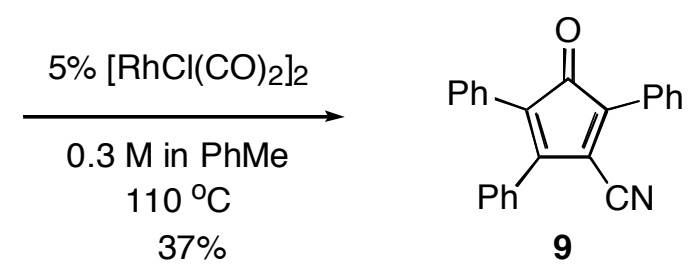

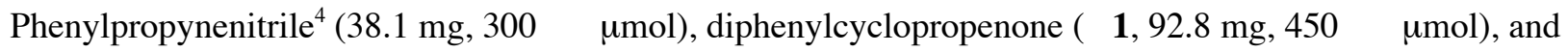
$\left[\mathrm{RhCl}(\mathrm{CO})_{2}\right]_{2}(15 \mu \mathrm{mol}, 5.8 \mathrm{mg})$ were weighed out into a test tube with stir bar under nitrogen. To this was added toluene (Solvtek ${ }^{\mathrm{TM}}, 900 \mu \mathrm{L}$ ). The tube was capped with a septum and heated in an oil bath for 11 hours at $110{ }^{\circ} \mathrm{C}$ under nitrogen. The resulting solution was purified by flash column chromatography eluting with $100 \mathrm{~mL}$ petroleum ether followed by $200 \mathrm{~mL} \mathrm{5 \%}$ diethyl ether/petroleum ether. Productcontaining fractions were collected and concentrated under reduced pressure to leave cyclopentadienone 9 as a purple solid $(36.6 \mathrm{mg}, 37 \%)$.

m. p. $128^{\circ} \mathrm{C}$.

${ }^{1} \mathbf{H}$ NMR $\left(400 \mathrm{MHz}, \mathrm{CDCl}_{3}\right)$ §: 7.98-7.96 (m, 2H), 7.52-7.42 (m, 8H), 7.30-7.22 (m, 5H).

${ }^{13} \mathrm{C} \mathrm{NMR}\left(\mathrm{CDCl}_{3}, 100 \mathrm{MHz}\right) \delta: 197.6,150.2,138.6,131.1,131.0,130.4,129.9$ (2C), 129.6 (2C), 129.3, 129.0 (2C), 128.9 (2C), 128.6 (2C), 128.4 (2C), 128.4, 128.3, 124.6, 122.1, 115.0.

FTIR (CDCl ${ }_{3}$ film): 3056 (w), 2210 (w), 1716 (s), 1621 (w), 1598 (w), 1574 (w), 1492 (w), 1444 (m), 1355 (w), 1297 (m), $1114(\mathrm{~m}), 1074(\mathrm{w}), 1028$ (w), $910(\mathrm{w}), 795$ (w), $761(\mathrm{w}), 727$ (s), $693(\mathrm{~s}) \mathrm{cm}^{-1}$.

HRMS for $\mathrm{C}_{24} \mathrm{H}_{15} \mathrm{NO}$ calc'd: $333.1154 \mathrm{~g} / \mathrm{mol}$, found: $333.1151 \mathrm{~g} / \mathrm{mol}$.

Elemental Analysis for $\mathrm{C}_{24} \mathrm{H}_{15} \mathrm{NO}$ calc'd: $86.46 \% \mathrm{C}, 4.54 \% \mathrm{H}, 4.20 \% \mathrm{~N}$; found $86.36 \% \mathrm{C}, 4.76 \% \mathrm{H}$, $4.09 \% \mathrm{~N}$. 


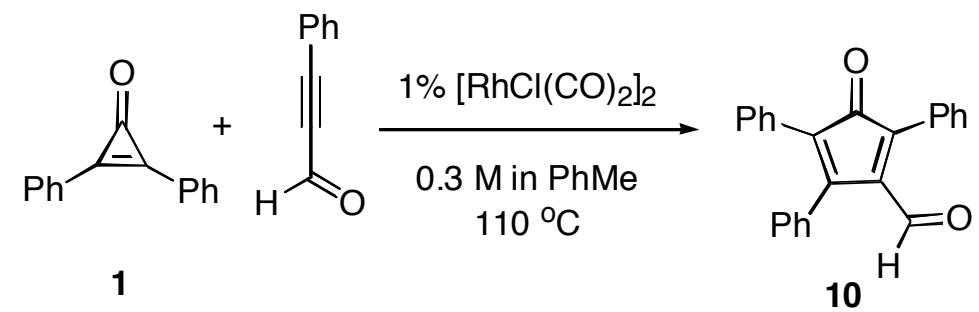

Cyclopentadienone 10:

Diphenylcyclopropenone (1, $77.3 \mathrm{mg}, 0.375 \mu \mathrm{mol})$ and 3-phenylpropynal $(32.6 \mathrm{mg}, 250 \mu \mathrm{mol})$ were weighed out into a test tube under nitrogen. To this was added toluene $\left(750 \mu \mathrm{L}\right.$, Solvtek $\left.{ }^{\mathrm{TM}}\right)$ and $\left[\mathrm{RhCl}(\mathrm{CO})_{2}\right]_{2}(1.0 \mathrm{mg}, 2.5 \mu \mathrm{mol})$. The tube was then capped with a septum and heated at $110{ }^{\circ} \mathrm{C}$ for 3 hours under nitrogen. The crude reaction mixture was then cooled to room temperature and then purified by flash column chromatography eluting with diethyl ether and pentane (1:19). Product-containing fractions were collected and concentrated under reduced pressure to give a purple solid $(35.4 \mathrm{mg}, 42 \%$ yield).

m. p. $171-173{ }^{\circ} \mathrm{C}$.

$\mathbf{R}_{\mathbf{f}}: 0.38$ in $24: 1$, pentane: ethyl acetate (appears purple in visible light).

${ }^{1} \mathbf{H}$ NMR $\left(\mathrm{CDCl}_{3}, 400 \mathrm{MHz}\right) \delta: 10.19(\mathrm{~s}, 1 \mathrm{H}), 7.59-7.54(\mathrm{~m}, 2 \mathrm{H}), 7.54-7.47$ (m, 3H), 7.42-7.32 (m, 3H), 7.26-7.21 (m, 5H), 7.19-7.14 (m, 2H).

${ }^{13} \mathbf{C}$ NMR $\left(\mathrm{CDCl}_{3}, 125 \mathrm{MHz}\right) \delta: 199.9,189.1,155.3,152.7,143.9,138.5,132.8,131.1$ (2C), 130.4, 130.0 (2C), 129.9, 129.1, 128.6 (2C), 128.5 (2C), 128.12 (2C), 128.09 (2C), 127.9, 126.6.

FTIR (thin film): 3054 (w), 2849 (w), 2360 (s), 2341 (s), 1713 (s), 1679 (m), 1598 (w), 1575 (w), 1489 (w), 1443 (m), 1354 (m), 1116 (m), 1074 (w), 1001 (w), 798 (m), 729 (m), 694 (s) cm².

HRMS for $\mathrm{C}_{24} \mathrm{H}_{16} \mathrm{O}_{2}$ calc'd: $336.1150 \mathrm{~g} / \mathrm{mol}$, found $336.1152 \mathrm{~g} / \mathrm{mol}$.<smiles>O=c1c(-c2ccccc2)c1-c1ccccc1</smiles>

1<smiles></smiles>

$\mathrm{Ph}$

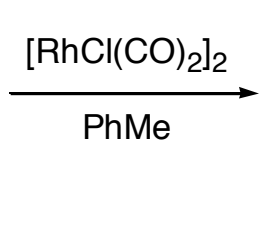<smiles>O=C1C(C#Cc2ccccc2)=C(c2ccccc2)C(c2ccccc2)=C1c1ccccc1</smiles>

11<smiles>O=C1C(c2ccccc2)=C(c2ccccc2)C(c2c(-c3ccccc3)c(-c3ccccc3)c(C#Cc3ccccc3)c(-c3ccccc3)c2-c2ccccc2)=C1c1ccccc1</smiles>

11a

Cyclopentadienones 11 and 11a:

Table 1. [3+2] Reactions of Diphenylbutadiyne.

\begin{tabular}{llll}
\hline Entry & Conditions & $\mathbf{1 1}$ & 11a \\
\hline 1 & $1 \mathrm{~mol} \%\left[\mathrm{RhCl}(\mathrm{CO})_{2}\right]_{2}, 9.5 \mathrm{~h}, 110{ }^{\circ} \mathrm{C}$ & $10 \%$ & $10 \%$ \\
2 & $1 \mathrm{~mol} \%\left[\mathrm{RhCl}(\mathrm{CO})_{2}\right]_{2}, 14.5 \mathrm{~h}, 80{ }^{\circ} \mathrm{C}$ & $5 \%$ & $17 \%$ \\
3 & $10 \mathrm{~mol} \%\left[\operatorname{RhCl}(\mathrm{CO})_{2}\right]_{2}, 21 \mathrm{~h}, 60{ }^{\circ} \mathrm{C}$ & $35 \%$ & Trace \\
\hline
\end{tabular}

Diphenylbutadiyne $(75.0 \mathrm{mg}, 371 \mu \mathrm{mol})$, diphenylcyclopropenone (1, $556 \mu \mathrm{mol}, 115 \mathrm{mg})$ and $\left[\mathrm{RhCl}(\mathrm{CO})_{2}\right]_{2}(3.7 \mu \mathrm{mol}, 1.4 \mathrm{mg})$ were weighed out into a test tube with stir bar under nitrogen. To this was added toluene (Solvtek ${ }^{\mathrm{TM}}, 1.1 \mathrm{~mL}$ ). The tube was capped with a septum and heated in an oil bath for 
9.5 hours at $110{ }^{\circ} \mathrm{C}$ under nitrogen. The resulting solution was purified by flash column chromatography eluting with $300 \mathrm{~mL} \mathrm{3 \%}$ diethyl ether/petroleum ether followed by $200 \mathrm{~mL} \mathrm{5 \%}$ then $100 \mathrm{~mL} 10 \%$. The resulting blue fraction (high $\mathrm{R}_{\mathrm{f}}$ ) was concentrated under reduced pressure to leave (11) as a blue solid $(15.1 \mathrm{mg}, 10 \%)$. The resulting brown fraction (low $\mathrm{R}_{\mathrm{f}}$ ) was concentrated under reduced pressure to leave (11a) as a blue solid (15.3 $\mathrm{mg}, 10 \%)$.

Data for 11:

m. p. $144-146{ }^{\circ} \mathrm{C}$.

${ }^{1}$ H NMR (400 MHz, $\left.\mathrm{CDCl}_{3}\right)$ \&: 8.13-8.10 (dd, 2H), 7.56-7.28 (m, 18H).

${ }^{13} \mathbf{C ~ N M R}\left(\mathrm{CDCl}_{3}, 100 \mathrm{MHz}\right) \delta: 200.1,151.1,135.5,132.6,131.7$ (2C), 131.1, 131.0, 130.4, 130.0 (2C), 129.9, 129.7, 129.4 (2C), 129.2, 129.1 (2C), 128.5 (2C), 128.3 (2C), 128.1 (2C), 128.1 (2C), 127.7, 125.0, $122.4,110.8,86.5$.

FTIR (CDCl ${ }_{3}$ film): 3056 (m), 2185 (m), 1705 (s), 1576 (w), 1599 (w), 1495 (m), 1486 (m), 1443 (m), $1365(\mathrm{~m}), 1327$ (m), 1128 (m), $1116(\mathrm{~m}), 1073(\mathrm{w}), 1006(\mathrm{w}), 910(\mathrm{~m}), 795(\mathrm{w}), 756(\mathrm{~m}), 727(\mathrm{~m}), 689$ (m) $\mathrm{cm}^{-1}$.

HRMS for $\mathrm{C}_{31} \mathrm{H}_{20} \mathrm{O}$ calc'd: $408.1514 \mathrm{~g} / \mathrm{mol}$, found: $408.1510 \mathrm{~g} / \mathrm{mol}$.

Data for 11a:

m. p. $300-302{ }^{\circ} \mathrm{C}$.

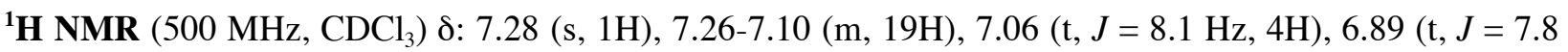
$\mathrm{Hz}, 4 \mathrm{H}), 6.75$ (apparent dd, $\left.J_{1}=13.9 \mathrm{~Hz}, J_{2}=7.8 \mathrm{~Hz}, 8 \mathrm{H}\right), 6.67$ (s, broad, 2H), 6.46 (s, broad, 2H).

${ }^{13} \mathbf{C}$ NMR $\left(\mathrm{CDCl}_{3}, 125 \mathrm{MHz}\right) \delta: 199.6,154.5,154.1,144.2,139.7,139.3,138.3,133.5,132.7,131.2$, 131.0, 130.9, 130.9, 130.1, 129.5, 129.1, 128.7, 128.0, 128.0, 127.9, 127.9, 127.6, 127.5, 127.2, 127.1, $127.0,127.0,126.5,126.5,126.2,125.0,124.6,123.1,98.1,89.2$. Because of dispersity problems in the region between 126 and 132 ppm, 8 carbon signals could not been unambiguously identified.

FTIR (CDCl ${ }_{3}$ film): 3056 (m), 2248 (w), 1947 (w), 1879 (w), 1707 (s), 1599 (m), 1492 (m), 1442 (m), $1405(\mathrm{w}), 1361(\mathrm{~m}), 1327(\mathrm{~m}), 1269$ (w), 1117 (m), $1074(\mathrm{~m}), 1027$ (m), 909 (s), 757 (m), 732 (s), $697(\mathrm{~s})$ $\mathrm{cm}^{-1}$.

MALDI for $\mathrm{C}_{61} \mathrm{H}_{40} \mathrm{O}$ calc'd: $788.3 \mathrm{~g} / \mathrm{mol}$, found: $790.4 \mathrm{~g} / \mathrm{mol}(\mathrm{M}+2 \mathrm{H}), 813.5 \mathrm{~g} / \mathrm{mol}(\mathrm{M}+\mathrm{H}+\mathrm{Na})$. 


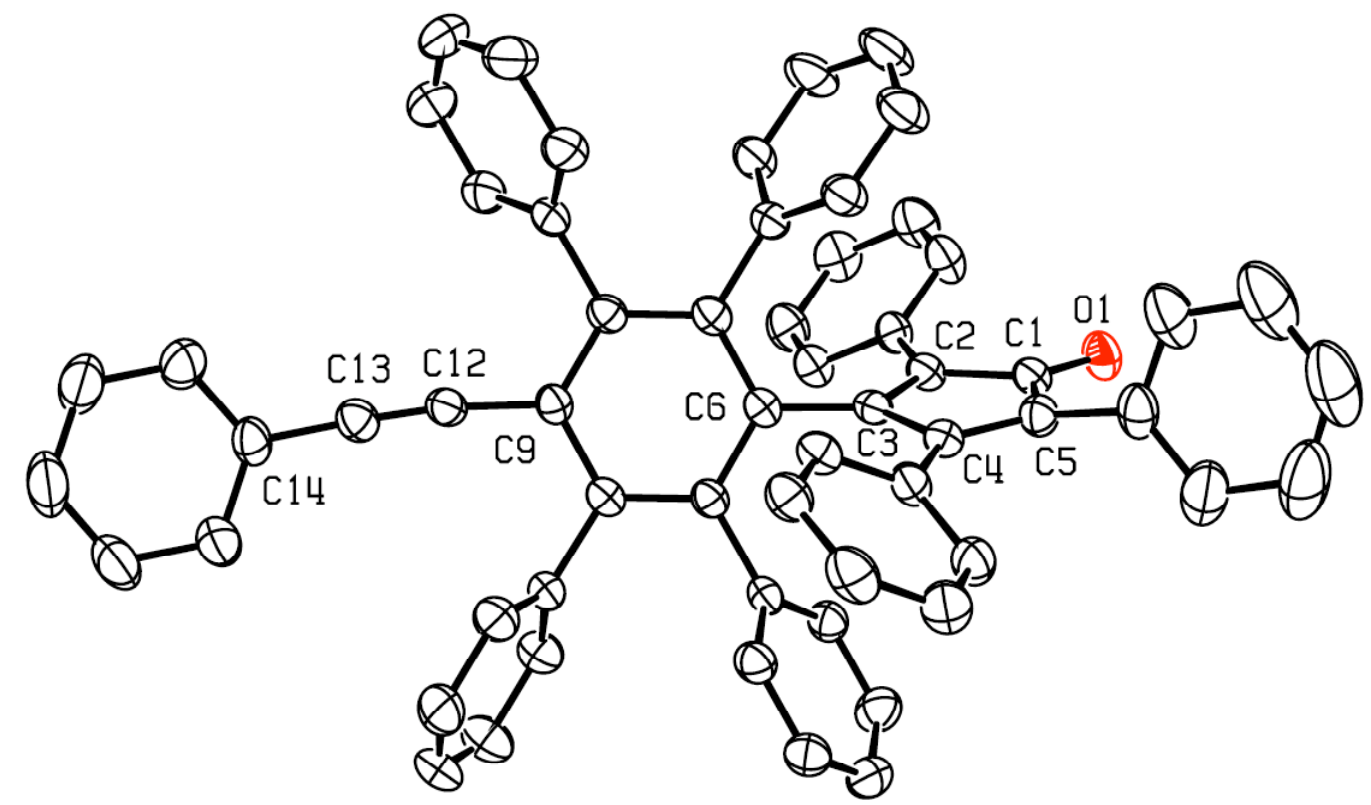

ORTEP diagram for 11a. Elipsoids drawn at the 50\% probability level.<smiles>O=c1c(-c2ccccc2)c1-c1ccccc1</smiles>

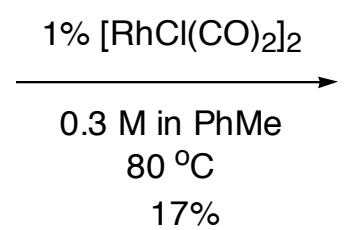<smiles>O=C1C(c2ccccc2)=C(c2ccccc2)C(c2ccccc2)=C1c1ccccc1</smiles>

Tetracyclone (12):

Diphenylacetylene $(44.6 \mathrm{mg}, 250 \mu \mathrm{mol})$, diphenylcyclopropenone $(1,250 \mu \mathrm{mol}, 53.1 \mathrm{mg})$ and $\left[\mathrm{RhCl}(\mathrm{CO})_{2}\right]_{2}(2.5 \mu \mathrm{mol}, 1.0 \mathrm{mg})$ were weighed out into a test tube with stir bar under nitrogen. To this was added toluene (Solvtek ${ }^{\mathrm{TM}}, 1.67 \mathrm{~mL}$ ). The tube was capped with a septum and heated in an oil bath for 65 hours at $80{ }^{\circ} \mathrm{C}$ under nitrogen. The resulting solution was purified by flash column chromatography eluting with $100 \mathrm{~mL}$ petroleum ether followed by $150 \mathrm{~mL} \mathrm{5 \%}$ diethyl ether/petroleum ether. Productcontaining fractions were collected and concentrated under reduced pressure to leave tetracyclone (12) as a purple solid (16.5 mg, 17\%).

m. p. $218-219{ }^{\circ} \mathrm{C}$.

${ }^{1} \mathbf{H}$ NMR $\left(400 \mathrm{MHz}, \mathrm{CDCl}_{3}\right)$ §: 7.25-7.23 (m, 12H), 7.19-7.16 (m, 4H), 6.94-6.92 (m, 4H).

${ }^{13} \mathbf{C ~ N M R}\left(\mathrm{CDCl}_{3}, 100 \mathrm{MHz}\right) \delta: 200.3,154.5$ (2C), 133.0 (2C), 130.7 (2C), 130.1 (4C), 129.3 (4C), 128.5 (2C), 128.0 (4C), 128.0 (4C), 127.4 (2C), 125.3 (2C).

FTIR (CDCl film): 3056 (w), 1711 (s), 1598 (w), 1488 (w), 1443 (m), 1354 (w), 1300 (m), 1116 (w), 1090 (w), 1075 (w), 1027 (w), 913 (w), 809 (m), 793 (w), 750 (w), 738 (w), 717 (s), 696 (s), $689(\mathrm{~s}) \mathrm{cm}^{-1}$.

HRMS for $\mathrm{C}_{29} \mathrm{H}_{20} \mathrm{O}$ calc'd: $384.1514 \mathrm{~g} / \mathrm{mol}$, found: $384.1515 \mathrm{~g} / \mathrm{mol}$. 


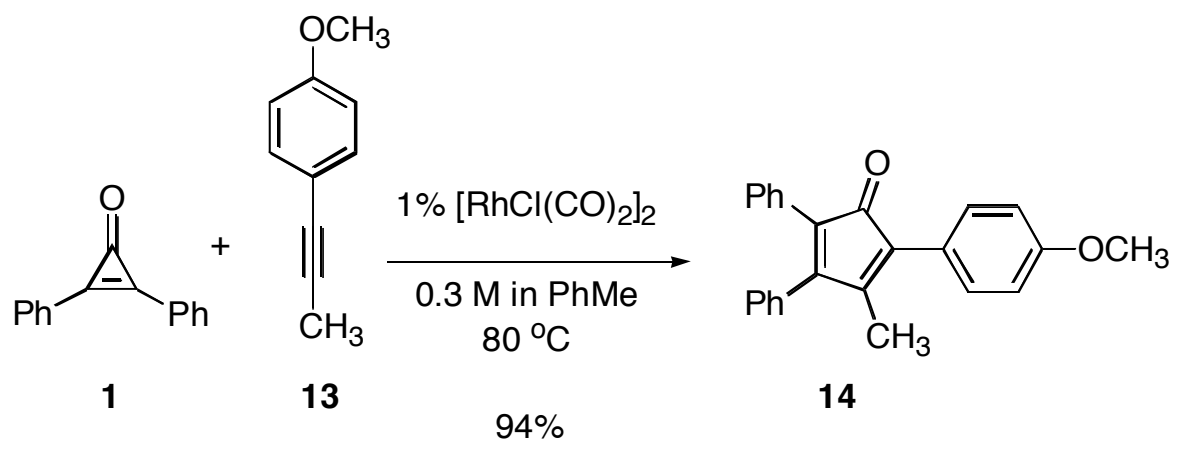

Cyclopentadienone 14:

Diphenylcyclopropenone (1, $79.4 \mathrm{mg}, 385 \mu \mathrm{mol})$ and alkyne $13^{5}(37.5 \mathrm{mg}, 257 \mu \mathrm{mol})$ were weighed out into a test tube with stir bar under nitrogen. To this was added toluene $\left(800 \mu \mathrm{L}\right.$, Solvtek $\left.{ }^{\mathrm{TM}}\right)$ and $\left[\mathrm{RhCl}(\mathrm{CO})_{2}\right]_{2}(1.0 \mathrm{mg}, 2.5 \mu \mathrm{mol})$. The tube was capped with a septum and heated at $80{ }^{\circ} \mathrm{C}$ for 2.5 hours under nitrogen. The crude reaction mixture was then cooled to room temperature and then purified by flash column chromatography eluting with diethyl ether and pentane (1:19). Product-containing fractions were collected and concentrated under reduced pressure to give $\mathbf{1 4}$ as a purple solid (85 $\mathrm{mg}, 94 \%$ yield).

m. p. $57-59{ }^{\circ} \mathrm{C}$.

$\mathbf{R}_{\mathbf{f}}$ : 0.31 in 19:1 pentane: ethyl acetate (appears purple in visible light).

${ }^{1} \mathbf{H}$ NMR $\left(\mathrm{CDCl}_{3}, 500 \mathrm{MHz}\right): \delta: ~ 7.44-7.36(\mathrm{~m}, 5 \mathrm{H}), 7.31-7.27(\mathrm{~m}, 2 \mathrm{H}), 7.25-7.16(\mathrm{~m}, 5 \mathrm{H})$, 7.01-6.97 (m, $2 \mathrm{H}), 3.85$ (s, 3H), $2.09(\mathrm{~s}, 3 \mathrm{H})$.

${ }^{13} \mathrm{C}$ NMR $\left(\mathrm{CDCl}_{3}, 125 \mathrm{MHz}\right) \delta: 200.9,158.9,154.9,152.4,133.9,130.8$ (2C), 129.8 (2C), 128.6 (3C), $128.5(2 \mathrm{C}), 127.9(2 \mathrm{C}), 127.2(2 \mathrm{C}), 125.2,124.3,123.8,113.8(2 \mathrm{C}), 55.2,14.5$.

FTIR (thin film): 3054 (w), 2933 (w), 2835 (s), 2361 (s), 1708 (s), 1603 (m), 1569 (w), 1509 (s), 1441 (m), $1285(\mathrm{~m}), 1249(\mathrm{~s}), 1179(\mathrm{~m}), 1112(\mathrm{w}), 1036(\mathrm{w}), 833(\mathrm{~m}), 697(\mathrm{~m}) \mathrm{cm}^{-1}$.

HRMS for $\mathrm{C}_{25} \mathrm{H}_{20} \mathrm{O}_{2}$ calc'd: $352.1463 \mathrm{~g} / \mathrm{mol}$, found $352.1466 \mathrm{~g} / \mathrm{mol}$.

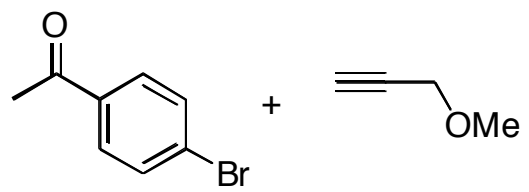

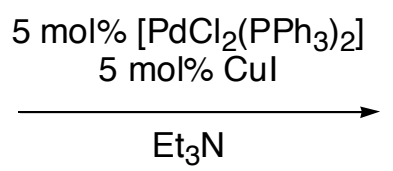

$68 \%$

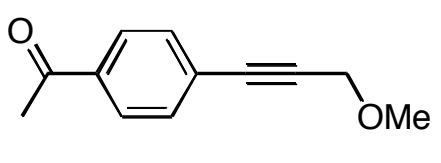

15

Alkyne 15:

Methyl propargyl ether $(0.489 \mathrm{~g}, 4.8 \mathrm{mmol}, 406 \mu \mathrm{L})$ was added to a stirring solution of 4'bromoacetophenone $(0.796 \mathrm{~g}, 4.0 \mathrm{mmol})$, copper(I) iodide $(38 \mathrm{mg}, 0.200 \mathrm{mmol})$, and bistriphenylphosphinopalladium(II) dichloride $(140 \mathrm{mg}, 0.200 \mathrm{mmol})$ in triethylamine $(12 \mathrm{~mL})$ under nitrogen. The suspension was stirred for 1 day at $50{ }^{\circ} \mathrm{C}$ under nitrogen and then poured over $25 \mathrm{~mL}$ water and $10 \mathrm{~mL}$ diethyl ether. The layers were separated and the aqueous layer was extracted with ether $(2 \times 20$ $\mathrm{mL}$ ). Combined organic fractions were washed with $1 \mathrm{~N} \mathrm{HCl}(\mathrm{aq})$, saturated $\mathrm{NaHCO}_{3}(\mathrm{aq})$, water, and brine $(30 \mathrm{~mL}$ each), dried over magnesium sulfate, filtered and concentrated by rotary evaporation. Further purification was accomplished by flash column chromatography eluting with $17 \%$ ether in pentane up to $33 \%$ ether in pentane. Product-containing fractions were concentrated to leave alkyne $\mathbf{1 5}$ (512 mg, 68\%). 
$\mathbf{R}_{\mathbf{f}}: 0.63$ in 1:1, pentane:ether (potassium permanganate stain).

${ }^{1}$ H NMR $\left(200 \mathrm{MHz}, \mathrm{CDCl}_{3}\right) \delta: 7.91(\mathrm{~d}, J=8.6 \mathrm{~Hz}, 2 \mathrm{H}), 7.53(\mathrm{~d}, J=8.6 \mathrm{~Hz}, 2 \mathrm{H}), 4.35(\mathrm{~s}, 2 \mathrm{H}), 3.47(\mathrm{~s}$, $3 \mathrm{H}), 2.60(\mathrm{~s}, 3 \mathrm{H})$.

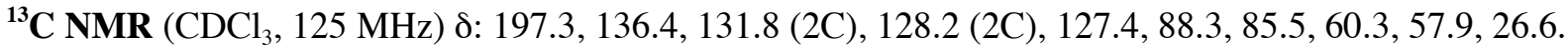

FTIR (CDCl ${ }_{3}$ film): 3042 (w), 2932 (m), 2822 (w), 1685 (s), 1602 (s), 1555 (w), 1404 (m), 1357 (m), 1264 (s), $1186(\mathrm{~m}), 1100(\mathrm{~s}), 958(\mathrm{~m}), 901(\mathrm{~m}), 838(\mathrm{~m}), 647(\mathrm{~m}) \mathrm{cm}^{-1}$.

HRMS for $\mathrm{C}_{12} \mathrm{H}_{12} \mathrm{O}_{2}$ calc'd: $188.0837 \mathrm{~g} / \mathrm{mol}$, found $188.0840 \mathrm{~g} / \mathrm{mol}$.

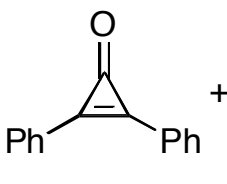

1

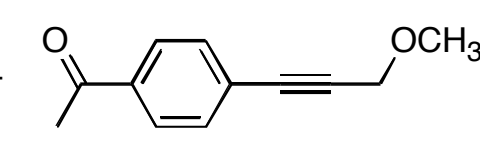

15

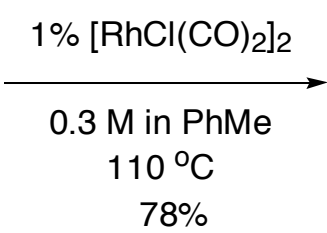

$78 \%$

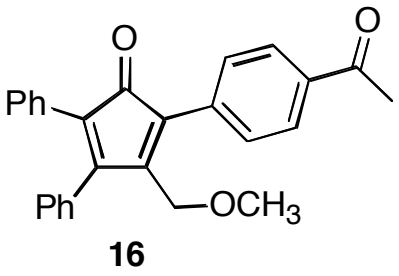

16

Cyclopentadienone 16:

Alkyne 15 (32.0 mg, $170 \mu \mathrm{mol})$, diphenylcyclopropenone (1, $53.0 \mathrm{mg}, 255 \mu \mathrm{mol})$, and $\left[\mathrm{RhCl}(\mathrm{CO})_{2}\right]_{2}(1.7$ $\mu \mathrm{mol}, 1.0 \mathrm{mg}$ ) were weighed out into a test tube with stir bar under nitrogen. To this was added toluene $\left(\right.$ Solvtek $^{\mathrm{TM}}, 510 \mu \mathrm{L}$ ). The tube was capped with a septum and heated in an oil bath for 5 hours at $110^{\circ} \mathrm{C}$ under nitrogen. The resulting solution was purified by flash column chromatography eluting with 100 $\mathrm{mL}$ pentane followed by $200 \mathrm{~mL} \mathrm{33 \%}$ diethyl ether/pentane. The product-containing fractions were concentrated under reduced pressure to leave cyclopentadienone $\mathbf{1 6}$ as a red solid (52.5 mg, 78\%).

m. p. $155-157^{\circ} \mathrm{C}$.

$\mathbf{R}_{\mathbf{f}}: 0.52$ in $3: 2$, pentane: ether (appears red in visible light).

${ }^{1}$ HNMR $\left(\mathrm{CDCl}_{3}, 500 \mathrm{MHz}\right):$ : 8.04-8.00 (m, 2H), 7.68-7.63 (m, 2H), 7.57-7.01 (m, 10H), $4.07(\mathrm{~s}, 2 \mathrm{H})$, $3.27(\mathrm{~s}, 3 \mathrm{H}), 2.59(\mathrm{~s}, 3 \mathrm{H})$.

${ }^{13} \mathrm{CNMR}\left(\mathrm{CDCl}_{3}, 50 \mathrm{MHz}\right): \delta: 200.0,197.7,155.3,152.0,136.3,135.2,133.1,130.5,130.4,129.9$ (2C), 129.8 (2C), 128.7 (2C), 128.5 (2C), 128.3 (2C), 128.1 (2C), 125.9, 125.3, 116.3, 65.5, 58.6, 26.6.

FTIR (thin film): 3407 (w), 3351 (w), 3058 (m), 2925 (m), 1738 (s), 1713 (s), $1682(\mathrm{~s}), 1601$ (m), 1485 (w), 1446 (m), 1356 (m), 1265 (m), 1170 (m), 1091 (m), 977 (m), 948 (m), 911 (m), 858 (m), 848 (m), $758(\mathrm{~m}), 732(\mathrm{~m}), 692(\mathrm{~m}) \mathrm{cm}^{-1}$.

HRMS for $\mathrm{C}_{27} \mathrm{H}_{22} \mathrm{O}_{3}$ calc'd: $394.1569 \mathrm{~g} / \mathrm{mol}$, found $394.1582 \mathrm{~g} / \mathrm{mol}$. 


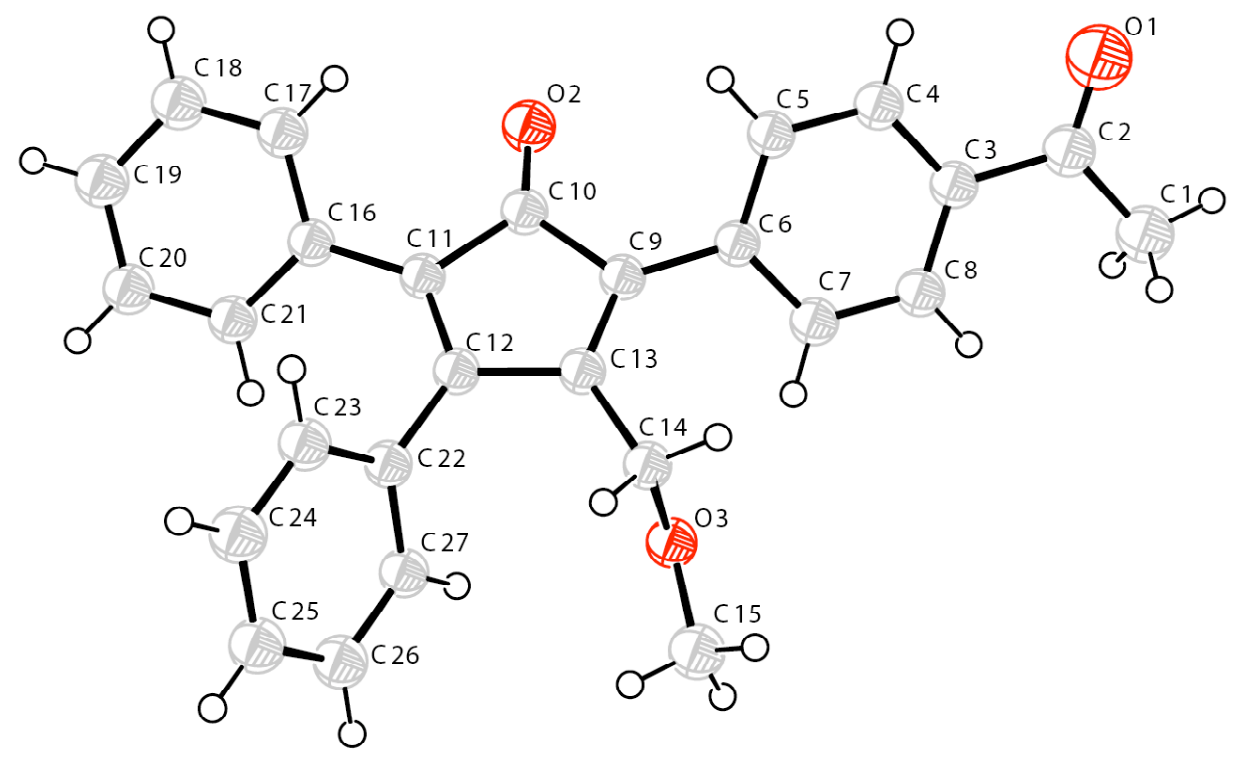

ORTEP diagram of 16. Ellipsoids drawn at the 50\% probability level.

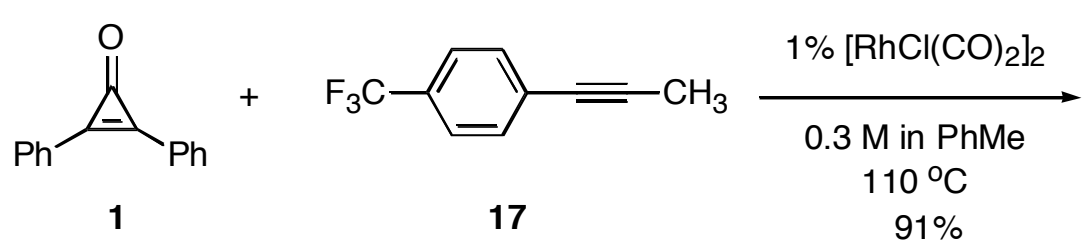<smiles>CC1=C(c2ccc(C(F)(F)F)cc2)C(=O)C(c2ccccc2)=C1c1ccccc1</smiles>

Cyclopentadienone 18:

4-Propynyltrifluoromethylbenzene ${ }^{6}(\mathbf{1 7}, 13.7 \mathrm{mg}, 74.4 \mu \mathrm{mol})$, diphenylcyclopropenone (1, $23.1 \mathrm{mg}, 112$ $\mu \mathrm{mol})$, and $\left[\mathrm{RhCl}(\mathrm{CO})_{2}\right]_{2}(0.744 \mu \mathrm{mol}, 0.5 \mathrm{mg})$ were weighed out into a test tube with stir bar under nitrogen. To this was added toluene (Solvtek ${ }^{\mathrm{TM}}, 410 \mu \mathrm{L}$ ). The tube was capped with a septum and heated in an oil bath for 6.5 hours at $110^{\circ} \mathrm{C}$ under nitrogen. The resulting solution was purified by flash column chromatography eluting with $10 \%$ diethyl ether/petroleum ether. Product-containing fractions were concentrated under reduced pressure to leave cyclopentadienone $\mathbf{1 8}$ as a red solid (26.3 $\mathrm{mg}, 91 \%)$.

m. p. $134-136^{\circ} \mathrm{C}$.

${ }^{1} \mathbf{H}$ NMR $\left(400 \mathrm{MHz}, \mathrm{CDCl}_{3}\right) \delta: 7.70(\mathrm{~d}, J=8.1 \mathrm{~Hz}, 2 \mathrm{H}), 7.57(\mathrm{~d}, J=8.1 \mathrm{~Hz}, 2 \mathrm{H}), 7.43-7.41(\mathrm{~m}, 3 \mathrm{H})$, 7.31-7.29 (m, 2H), 7.26-7.21 (m, 5H), $2.13(\mathrm{~s}, 3 \mathrm{H})$.

${ }^{13} \mathbf{C}$ NMR $\left(\mathrm{CDCl}_{3}, 100 \mathrm{MHz}\right) \delta: 199.8,156.2,154.2,135.0$ (q, $\left.J_{\mathrm{CF}}=1.5 \mathrm{~Hz}\right), 133.4,130.4,129.9$ (2C), $129.8(2 \mathrm{C}), 129.1\left(\mathrm{q}, J_{\mathrm{CF}}=32.8 \mathrm{~Hz}\right), 128.8(\mathrm{~m}, 3 \mathrm{C}), 128.7,128.6(2 \mathrm{C}), 128.1,(2 \mathrm{C}), 127.6,125.2\left(\mathrm{q}, J_{\mathrm{CF}}=\right.$ $3.8 \mathrm{~Hz}, 2 \mathrm{C}), 124.5,124.2\left(\mathrm{q}, J_{\mathrm{CF}}=271.6 \mathrm{~Hz}\right), 14.7$.

FTIR $\left(\mathrm{CDCl}_{3}\right.$ film): 3058 (m), 2926 (m), 2853 (w), 1713 (s), 1614 (s), 1485 (m), 1443 (m), 1408 (m), 1380 (m), 1324 (s), 1166 (s), 1121 (s), 1068 (s), 1018 (s), 993 (m), 945 (w), 915 (w), 842 (s), 794 (s), 774 (s), $738(\mathrm{~s}), 702(\mathrm{~s}) \mathrm{cm}^{-1}$.

HRMS for $\mathrm{C}_{25} \mathrm{H}_{17} \mathrm{~F}_{3} \mathrm{O}$ calc'd: $390.1232 \mathrm{~g} / \mathrm{mol}$, found: $390.1229 \mathrm{~g} / \mathrm{mol}$.

Elemental Analysis for $\mathrm{C}_{25} \mathrm{H}_{17} \mathrm{~F}_{3} \mathrm{O}$ calc'd: $76.91 \% \mathrm{C}, 4.39 \% \mathrm{H}, 14.60 \% \mathrm{~F}$; found $76.41 \% \mathrm{C}, 4.70 \% \mathrm{H}$, $14.50 \% \mathrm{~F}$. 


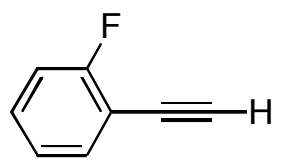

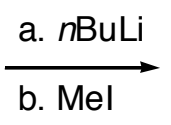

$73 \%$

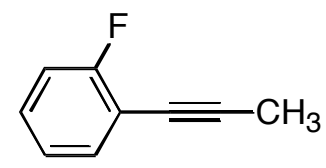

19

2-Propynylfluorobenzene (19):

2-Ethynylfluorobenzene $(120 \mathrm{mg}, 1.00 \mathrm{mmol})$ was weighed out into a test tube with a stir bar under nitrogen and capped with a septum. Tetrahydrofuran (Solvtek ${ }^{\mathrm{TM}}, 3.0 \mathrm{~mL}$ ) was added and the solution was cooled to $-78{ }^{\circ} \mathrm{C}$. $n$-Butyllithium $(2.5 \mathrm{M}$ in hexanes, $800 \mu \mathrm{L}, 2.00 \mathrm{mmol})$ was added and the reaction was stirred for 10 minutes. Iodomethane $(710 \mathrm{mg}, 312 \mu \mathrm{L}, 5.00 \mathrm{mmol})$ was then added and the reaction was warmed to room temperature. Saturated aqueous sodium thiosulfate $(10 \mathrm{~mL})$ was added, and the organic layer was extracted. The aqueous layer was extracted once with $10 \mathrm{~mL}$ pentane, the combined organic layers were concentrated and purified by flash column chromatography eluting with pentane. Productcontaining fractions were identified and concentrated under reduced pressure to yield 2propynylfluorobenzene $(\mathbf{1 9}, 98.3 \mathrm{mg}, \mathbf{7 3 \%})$.

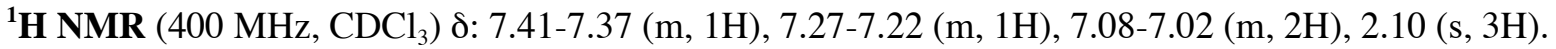

${ }^{13} \mathbf{C ~ N M R}\left(\mathrm{CDCl}_{3}, 100 \mathrm{MHz}\right) \delta: 162.8\left(\mathrm{~d}, J_{\mathrm{CF}}=250.2 \mathrm{~Hz}\right), 133.5,129.1\left(\mathrm{~d}, J_{\mathrm{CF}}=7.6 \mathrm{~Hz}\right), 123.8\left(\mathrm{~d}, J_{\mathrm{CF}}=\right.$ $3.8 \mathrm{~Hz}), 115.3\left(\mathrm{~d}, J_{\mathrm{CF}}=21.4 \mathrm{~Hz}\right), 112.4\left(\mathrm{~d}, J_{\mathrm{CF}}=16.0 \mathrm{~Hz}\right), 91.3\left(\mathrm{~d}, J_{\mathrm{CF}} 3.1=\mathrm{Hz}\right), 73.0,4.6$.

FTIR (CDCl film): 2918 (m), 2849 (w), 2242 (w), 1575 (w), 1492 (s), $1446(\mathrm{~m}), 1259$ (m), $1216(\mathrm{~m})$, $1154(\mathrm{w}), 1102(\mathrm{~m}), 1029(\mathrm{~m}), 941(\mathrm{w}), 817(\mathrm{~m}), 755(\mathrm{~s}) \mathrm{cm}^{-1}$.

HRMS for $\mathrm{C}_{9} \mathrm{H}_{7} \mathrm{~F}$ calc'd: $134.0528 \mathrm{~g} / \mathrm{mol}$, found: $134.0532 \mathrm{~g} / \mathrm{mol}$.

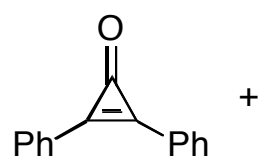

1

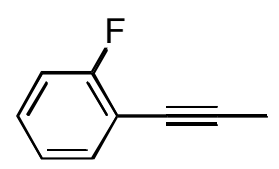

19

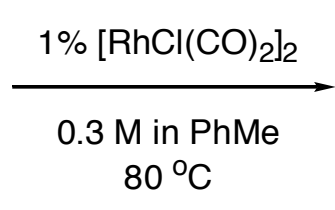

$77 \%$

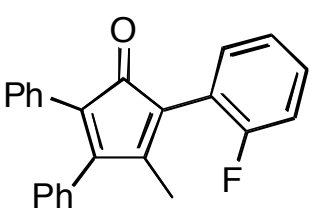

20

Cyclopentadienone 20:

2-Propynylfluorobenzene (19, $46.3 \mathrm{mg}, 345 \mu \mathrm{mol})$, diphenylcyclopropenone (1, $100 \mathrm{mg}, 487 \mu \mathrm{mol})$, and $\left[\mathrm{RhCl}(\mathrm{CO})_{2}\right]_{2}(3.45 \mu \mathrm{mol}, 1.3 \mathrm{mg})$ were weighed out into a test tube with stir bar under nitrogen. To this was added toluene (Solvtek ${ }^{\mathrm{TM}}, 975 \mu \mathrm{L}$ ). The tube was capped with a septum and heated in an oil bath for 20 hours at $80{ }^{\circ} \mathrm{C}$ under nitrogen. The resulting solution was purified by flash column chromatography

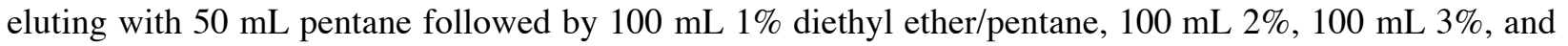
$50 \mathrm{~mL} 4 \%$. Product-containing fractions were identified and concentrated under reduced pressure to leave cyclopentadienone $\mathbf{2 0}$ as a red solid $(90.0 \mathrm{mg}, \mathbf{7 7 \%})$.

m. p. $133-135{ }^{\circ} \mathrm{C}$.

${ }^{1}$ H NMR (400 MHz, $\left.\mathrm{CDCl}_{3}\right)$ §: 7.43-7.14 (m, 14H), 2.00 (s, 3H).

${ }^{13} \mathbf{C ~ N M R}\left(\mathrm{CDCl}_{3}, 125 \mathrm{MHz}\right) \delta: 199.4,160.3\left(\mathrm{~d}, J_{\mathrm{CF}}=247.8 \mathrm{~Hz}\right), 157.4,153.9,133.5,131.9\left(\mathrm{~d}, J_{\mathrm{CF}}=3.0\right.$ $\mathrm{Hz}), 130.6,129.9,129.5$ (d, $\left.J_{\mathrm{CF}}=8.1 \mathrm{~Hz}\right), 128.7(2 \mathrm{C}), 128.6(2 \mathrm{C}), 128.6,128.0(2 \mathrm{C}), 127.4,125.4,123.9$ $\left(\mathrm{d}, J_{\mathrm{CF}}=3.0 \mathrm{~Hz}\right), 121.0,119.1\left(\mathrm{~d}, J_{\mathrm{CF}}=16.1 \mathrm{~Hz}, 2 \mathrm{C}\right), 115.8\left(\mathrm{~d}, J_{\mathrm{CF}}=22.7 \mathrm{~Hz}\right), 15.1\left(\mathrm{~d}, J_{\mathrm{CF}}=4.5 \mathrm{~Hz}\right)$. 


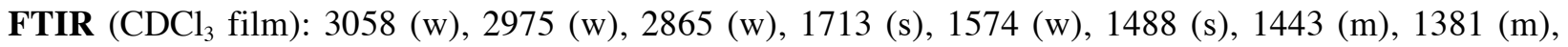
1347 (w), 1303 (m), 1261 (w), 1214 (m), 1118 (m), 1099 (m), 1073 (w), 1027 (w), 1000 (w), 993 (w), $913(\mathrm{w}), 824(\mathrm{w}), 793(\mathrm{~m}), 755(\mathrm{~s}), 741(\mathrm{w}), 696(\mathrm{~s}) \mathrm{cm}^{-1}$.

HRMS for $\mathrm{C}_{24} \mathrm{H}_{17} \mathrm{FO}$ calc'd: $340.1264 \mathrm{~g} / \mathrm{mol}$, found: $340.1260 \mathrm{~g} / \mathrm{mol}$.

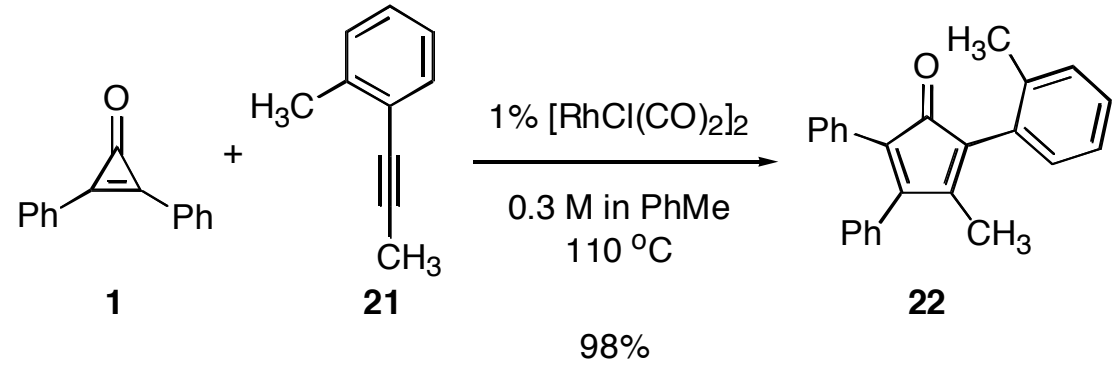

Cyclopentadienone 22:

Diphenylcyclopropenone $(\mathbf{1}, 87.9 \mathrm{mg}, 426 \mu \mathrm{mol})$ and alkyne 21 (37 mg, $284 \mu \mathrm{mol})$ were weighed out into a test tube with stir bar under nitrogen. To this was added toluene $\left(860 \mu \mathrm{L}\right.$, Solvtek $\left.{ }^{\mathrm{TM}}\right)$ and $\left[\mathrm{RhCl}(\mathrm{CO})_{2}\right]_{2}$ $(1.0 \mathrm{mg}, 2.5 \mu \mathrm{mol})$. The tube was capped with a septum and the reaction was heated at $110{ }^{\circ} \mathrm{C}$ for 5 hours under nitrogen. The crude reaction mixture was then cooled to room temperature and purified by flash column chromatography eluting with diethyl ether and pentane (1:19). Product-containing fractions were collected and concentrated under reduced pressure to give $\mathbf{2 2}$ as a purple solid (94 $\mathrm{mg}, \mathbf{9 8 \%}$ yield).

m. p. $50-53{ }^{\circ} \mathrm{C}$.

$\mathbf{R}_{\mathbf{f}}: 0.52$ in 24:1, pentane: ethyl acetate (appears red in visible light).

${ }^{1} \mathbf{H}$ NMR $\left(\mathrm{CDCl}_{3}, 400 \mathrm{MHz}\right)$ : $\delta: ~ 7.42-7.34(\mathrm{~m}, 2 \mathrm{H})$, 7.32-7.12 (m, 11H), 6.94-6.91 (m, 1H), $2.27(\mathrm{~s}, 3 \mathrm{H})$, $1.88(\mathrm{~s}, 3 \mathrm{H})$.

${ }^{13} \mathbf{C}$ NMR $\left(\mathrm{CDCl}_{3}, 125 \mathrm{MHz}\right) \delta:$ 200.2, 155.4, 153.6, 137.5, 133.7, 131.1, 130.7, 130.4, 130.2, 130.1, 129.8 (2C), 129.3, 128.6 (2C), 128.5, 128.0 (2C), 127.9 (2C), 127.3, 125.5, 124.9, 20.5, 14.5.

FTIR (thin film): 3056 (w), 3018 (w), 2923 (w), 1710 (s), 1486 (m), 1443 (m), 1380 (w), 1345 (w), 1301 (w), $990(\mathrm{w}), 910(\mathrm{w}), 795(\mathrm{w}), 747(\mathrm{~m}), 738(\mathrm{~m}), 696(\mathrm{~s}) \mathrm{cm}^{-1}$.

HRMS for $\mathrm{C}_{25} \mathrm{H}_{20} \mathrm{O}$ calc'd: $336.1514 \mathrm{~g} / \mathrm{mol}$, found $336.1510 \mathrm{~g} / \mathrm{mol}$.

Elemental Analysis for $\mathrm{C}_{25} \mathrm{H}_{20} \mathrm{O}$ calc'd: $89.25 \% \mathrm{C} ; 5.99 \% \mathrm{H} ; 4.76 \%$ O; found $89.04 \% \mathrm{C} ; 6.10 \% \mathrm{H}$.
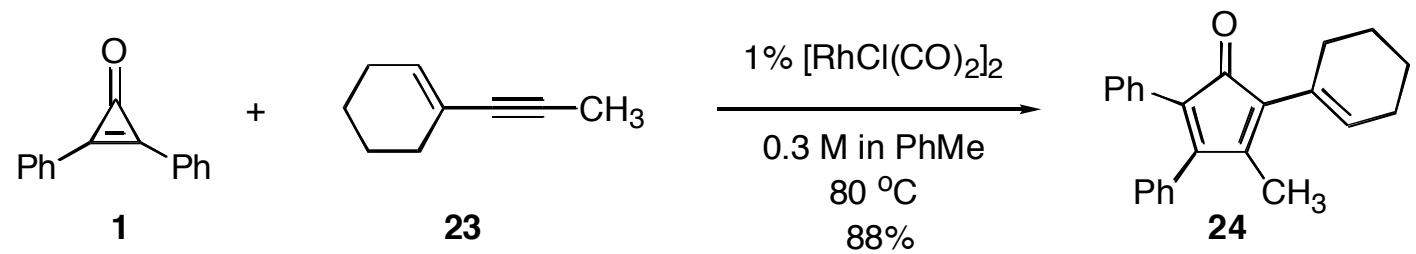

Cyclopentadienone 24:

1-propynylcyclohexene ${ }^{7}$ (23, 35.0 mg, $291 \mu \mathrm{mol}$ ), diphenylcyclopropenone (1, $\left.90.1 \mathrm{mg}, 437 \mu \mathrm{mol}\right)$, and $\left[\mathrm{RhCl}(\mathrm{CO})_{2}\right]_{2}(1.1 \mathrm{mg}, 2.9 \mu \mathrm{mol})$ were weighed out into a test tube with stir bar under nitrogen. To this was added toluene (Solvtek ${ }^{\mathrm{TM}}, 874 \mu \mathrm{L}$ ). The tube was capped with a septum and heated in an oil bath for 3 hours at $80{ }^{\circ} \mathrm{C}$ under nitrogen. The resulting solution was purified by flash column chromatography eluting with $100 \mathrm{~mL}$ petroleum ether followed by $200 \mathrm{~mL}$ 5\% diethyl ether/petroleum ether. Product- 
containing fractions were collected and concentrated under reduced pressure to leave cyclopentadienone 24 as a red solid $(83.7 \mathrm{mg}, 88 \%)$.

m. p. $85-88^{\circ} \mathrm{C}$.

${ }^{1} \mathbf{H}$ NMR $\left(500 \mathrm{MHz}, \mathrm{CDCl}_{3}\right)$ 8: 7.40-7.37 (m, 3H), 7.26-7.24 (m, 2H), 7.20-7.17 (m, 5H), $5.82\left(\mathrm{dd}, J_{1}=\right.$ $\left.3.8 \mathrm{~Hz}, J_{2}=2.0 \mathrm{~Hz}, 1 \mathrm{H}\right), 2.34-2.31(\mathrm{~m}, 2 \mathrm{H}), 2.24-2.22(\mathrm{~m}, 2 \mathrm{H}), 1.98(\mathrm{~s}, 3 \mathrm{H}), 1.77-1.74(\mathrm{~m}, 2 \mathrm{H}), 1.73-$ $1.68(\mathrm{~m}, 2 \mathrm{H})$; assigned by COSY.

${ }^{13} \mathrm{C}$ NMR $\left(\mathrm{CDCl}_{3}, 125 \mathrm{MHz}\right) \delta: 201.4,154.6,151.7,134.1,130.9,129.8,129.7$ (2C), 129.7, 128.6 (2C), $128.6(2 \mathrm{C}), 128.5,128.3,127.9(2 \mathrm{C}), 127.0,124.3,28.2,25.7,22.8,22.0,14.5$.

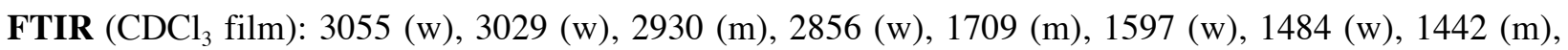
$1380(\mathrm{~m}), 1347(\mathrm{~m}), 1288(\mathrm{~m}), 1264(\mathrm{w}), 1157(\mathrm{w}), 1136(\mathrm{w}), 1101(\mathrm{~m}), 1072(\mathrm{w}), 1000(\mathrm{w}), 911(\mathrm{~m})$, $855(\mathrm{w}), 790(\mathrm{~m}), 768(\mathrm{~m}), 733(\mathrm{~m}), 715(\mathrm{~m}), 699(\mathrm{~s}) \mathrm{cm}^{-1}$.

HRMS for $\mathrm{C}_{24} \mathrm{H}_{22} \mathrm{O}$ calc'd: $326.1671 \mathrm{~g} / \mathrm{mol}$, found: $326.1673 \mathrm{~g} / \mathrm{mol}$.

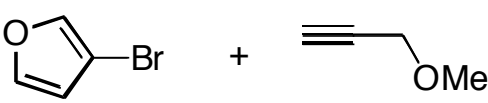

3-(3-Methoxypropynyl)furan (25):

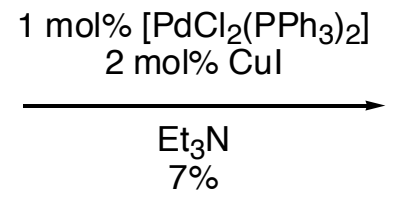

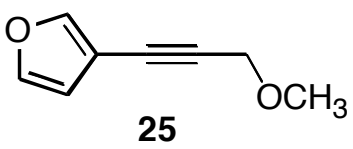

3-Bromofuran $(2.21 \mathrm{~g}, 15.0 \mathrm{mmol})$ was weighed out in an oven-dried $50 \mathrm{~mL}$ round bottom flask with a stir bar. Triethylamine $(5 \mathrm{~mL})$, methyl propargyl ether $(1.58 \mathrm{~g}, 22.5 \mathrm{mmol}, 1.90 \mathrm{~mL})$, copper(I) iodide (57.2 $\mathrm{mg}, 0.300 \mathrm{mmol}$ ), and bistriphenylphosphinopalladium(II) dichloride (106 $\mathrm{mg}, 300 \mathrm{mmol})$ were then added and the flask was flushed with nitrogen. The suspension was stirred for 2 days at $50{ }^{\circ} \mathrm{C}$ under nitrogen and then poured over $20 \mathrm{~mL}$ saturated ammonium chloride and $10 \mathrm{~mL}$ diethyl ether. The organic layer was extracted and the aqueous layer was extracted with ether $(2 \times 10 \mathrm{~mL})$. Combined organic fractions were dried over sodium sulfate and filtered through fluorosil. The resulting solution was concentrated by rotary evaporation and then purified by flash column chromatography eluting with 200 $\mathrm{mL}$ pentane, $200 \mathrm{~mL} \mathrm{5 \%}$ diethyl ether/pentane, followed by $100 \mathrm{~mL} \mathrm{10 \%} \mathrm{diethyl} \mathrm{ether/pentane.} \mathrm{Product-}$ containing fractions were identified and concentrated to yield furan 25 (149 mg, 7\%).

${ }^{1} \mathbf{H}$ NMR $\left(500 \mathrm{MHz}, \mathrm{CDCl}_{3}\right) \delta: 7.62(\mathrm{~m}, 1 \mathrm{H}), 7.36\left(\mathrm{dd}, J_{1}=J_{2}=1.7 \mathrm{~Hz}, 1 \mathrm{H}\right), 6.44\left(\mathrm{dd}, J_{1}=1.9 \mathrm{~Hz}, J_{2}=\right.$ $0.8 \mathrm{~Hz}, 1 \mathrm{H}), 4.28$ (s, 2H), 3.43 (s, 3H).

${ }^{13} \mathbf{C}$ NMR $\left(\mathrm{CDCl}_{3}, 125 \mathrm{MHz}\right) \delta: 145.9,142.8,112.5,107.0,86.8,77.6,60.4,57.7$.

FTIR (CDCl ${ }_{3}$ film): 3147 (w), 2929 (m), 2822 (w), 2233 (w), 1450 (w), 1357 (m), $1286(w), 1187$ (w), $1163(\mathrm{~m}), 1100(\mathrm{~s}), 1031(\mathrm{~m}), 1006(\mathrm{~m}), 871(\mathrm{~m}), 792(\mathrm{~m}) \mathrm{cm}^{-1}$.

HRMS for $\mathrm{C}_{8} \mathrm{H}_{8} \mathrm{O}_{2}$ calc'd: $136.054230 \mathrm{~g} / \mathrm{mol}$, found: $136.052513 \mathrm{~g} / \mathrm{mol}$.
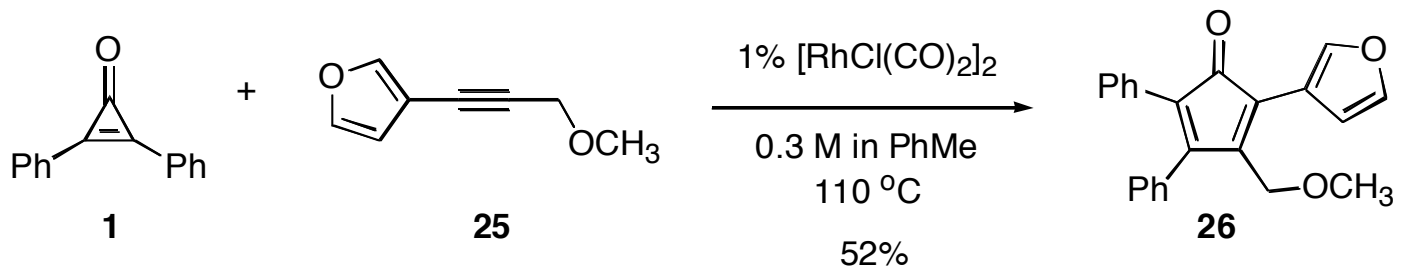

Cyclopentadienone 26: 
Furan $(\mathbf{2 5}, 27.1 \mathrm{mg}, 199 \mu \mathrm{mol})$, diphenylcyclopropenone $(\mathbf{1}, 61.5 \mathrm{mg}, 299 \mu \mathrm{mol})$, and $\left[\mathrm{RhCl}(\mathrm{CO})_{2}\right]_{2}(2.0$ $\mu \mathrm{mol}, 0.8 \mathrm{mg}$ ) were weighed out into a test tube with stir bar under nitrogen. To this was added toluene $\left(\right.$ Solvtek $\left.^{\mathrm{TM}}, 600 \mu \mathrm{L}\right)$. The tube was capped with a septum and heated in an oil bath for 17.5 hours at 110 ${ }^{\circ} \mathrm{C}$ under nitrogen. The resulting solution was purified twice by flash column chromatography eluting with 5\% diethyl ether/ petroleum ether. Product-containing fractions were identified and concentrated under reduced pressure to leave cyclopentadienone $\mathbf{2 6}$ as a purple solid (35.4 $\mathrm{mg}, \mathbf{5 2 \%}$ ).

m. p. $128-131{ }^{\circ} \mathrm{C}$.

${ }^{1} \mathbf{H}$ NMR $\left(500 \mathrm{MHz}, \mathrm{CDCl}_{3}\right)$ ): 7.99-7.98 (m, 1H), $7.49(\mathrm{~m}, 1 \mathrm{H}), 7.40-7.35(\mathrm{~m}, 5 \mathrm{H})$, 7.22-7.17 (m, 5H), 6.80-6.79 (m, 1H), $4.14(\mathrm{~s}, 2 \mathrm{H}), 3.37(\mathrm{~s}, 3 \mathrm{H})$.

${ }^{13} \mathbf{C}$ NMR $\left(\mathrm{CDCl}_{3}, 125 \mathrm{MHz}\right) \delta: 200.5,156.5,146.7,143.2,143.1,133.3,130.7,129.7$ (2C), 128.9, 128.7 (2C), 128.4 (2C), 128.0 (2C), 127.3, 124.8, 122.2, 115.7, 110.0, 65.3, 58.5.

FTIR (CDCl ${ }_{3}$ film): 2923 (m), 1713 (s), 1443 (m), 1346 (w), 1306 (w), 1159 (m), 1126 (m), 1089 (m), $1025(\mathrm{w}), 945(\mathrm{~m}), 871(\mathrm{~m}), 803(\mathrm{~m}), 777(\mathrm{w}), 730(\mathrm{~m}), 695(\mathrm{~m}) \mathrm{cm}^{-1}$.

HRMS for $\mathrm{C}_{23} \mathrm{H}_{18} \mathrm{O}_{3}$ calc'd: $342.125595 \mathrm{~g} / \mathrm{mol}$, found: $342.126090 \mathrm{~g} / \mathrm{mol}$.

Elemental Analysis for $\mathrm{C}_{23} \mathrm{H}_{18} \mathrm{O}_{3}$ calc'd: $80.68 \%$ C, $5.30 \% \mathrm{H}, 14.02 \% \mathrm{O}$; found $80.79 \% \mathrm{C}, 5.52 \% \mathrm{H}$.

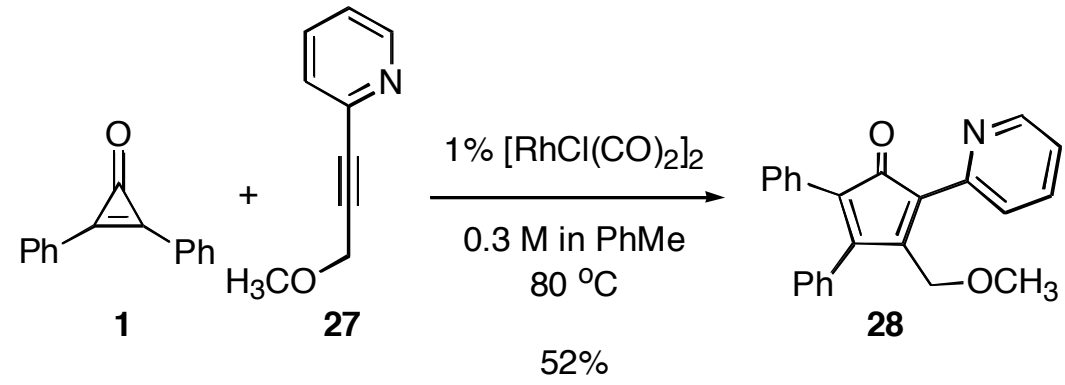

Cyclopentadienone 28:

Diphenylcyclopropenone (1, $70.8 \mathrm{mg}, 345 \mu \mathrm{mol})$ and alkyne $27^{8}(33.7 \mathrm{mg}, 229 \mu \mathrm{mol})$ were weighed out into a test tube with stir bar under nitrogen. To this was added toluene (Solvtek $\left.{ }^{\mathrm{TM}}, 690 \mu \mathrm{L}\right)$ and $\left[\mathrm{RhCl}(\mathrm{CO})_{2}\right]_{2}(1.0 \mathrm{mg}, 2.5 \mu \mathrm{mol})$. The tube was capped with a septum and then heated at $80{ }^{\circ} \mathrm{C}$ for 20 hours under nitrogen. The crude reaction mixture was then cooled to room temperature and purified by flash column chromatography eluting with diethyl ether and pentane (2:3). Product-containing fractions were collected and concentrated under reduced pressure to give $\mathbf{2 8}$ as a yellow solid ( $42 \mathrm{mg}, 52 \%$ yield).

m. p. $81-83^{\circ} \mathrm{C}$.

$\mathbf{R}_{\mathbf{f}}: 0.39$ in $3: 2$, pentane: ethyl acetate (appears yellow in visible light).

${ }^{1} \mathbf{H}$ NMR $\left(\mathrm{CDCl}_{3}, 400 \mathrm{MHz}\right) \delta:$ 7.52-7.42 (m, 3H), 7.39-7.34 (m, 2H), 7.19-7.11 (m, 5H), $6.37(\mathrm{dq}, J=$ $7.2 \mathrm{~Hz}, J=0.6 \mathrm{~Hz}, 1 \mathrm{H}), 6.08(\mathrm{qd}, J=4.8 \mathrm{~Hz}, J=0.5 \mathrm{~Hz}, 1 \mathrm{H}), 6.03(\mathrm{dt}, J=8.8 \mathrm{~Hz}, J=1.3 \mathrm{~Hz}, 1 \mathrm{H}), 5.44$ $(\mathrm{qd}, J=4.1 \mathrm{~Hz}, J=1.5 \mathrm{~Hz}, 1 \mathrm{H}), 4.17(\mathrm{~d}, J=16 \mathrm{~Hz}, 1 \mathrm{H}), 4.12(\mathrm{~d}, J=16 \mathrm{~Hz}, 1 \mathrm{H}), 3.35(\mathrm{~s}, 3 \mathrm{H})$.

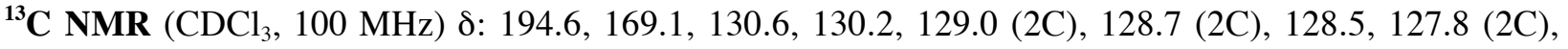
126.2 (2C), 123.8, 123.6, 119.9, 110.2, 108.9, 82.4, 81.4, 77.2, 62.5, 59.9, 57.5.

FTIR (thin film): 3369 (w), 3055 (w), 2929 (w), 2360 (m), 2341 (m), 1845 (w), 1685 (s), 1602 (m), 1581 (m), 1543 (s), 1448 (m), $1419(\mathrm{~m}), 1358(\mathrm{~m}), 1323(\mathrm{~m}), 1098(\mathrm{~s}), 1009(\mathrm{~m}), 756(\mathrm{~m}), 697(\mathrm{~m}) \mathrm{cm}^{-1}$. 
HRMS for $\mathrm{C}_{24} \mathrm{H}_{19} \mathrm{NO}_{2}$ calc'd: $353.1416 \mathrm{~g} / \mathrm{mol}$, found $353.1400 \mathrm{~g} / \mathrm{mol}$.

Elemental Analysis for $\mathrm{C}_{24} \mathrm{H}_{19} \mathrm{NO}_{2}$ calc'd: $81.56 \%$ C, $5.42 \% \mathrm{H}, 3.96 \% \mathrm{~N}$; found $81.51 \% \mathrm{C}, 5.10 \% \mathrm{H}$, $3.83 \% \mathrm{~N}$.

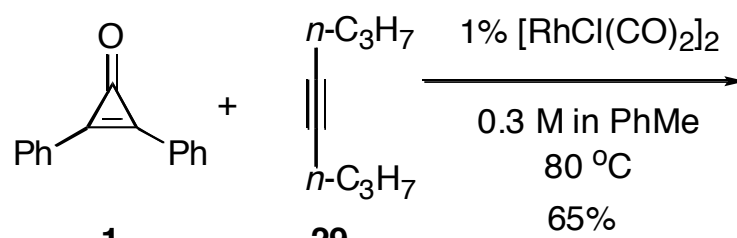

1<smiles>O=C1C(c2ccccc2)=C(Pc2ccccc2)C(c2ccccc2)=C1c1ccccc1</smiles>

30

Cyclopentadienone 30:

Diphenylcyclopropenone (1,53.0 mg, $256 \mu \mathrm{mol})$, 4-octyne $(\mathbf{2 9}, 18.8 \mathrm{mg}, 170 \mu \mathrm{mol})$, and $\left[\mathrm{RhCl}(\mathrm{CO})_{2}\right]_{2}$ $(1.0 \mathrm{mg}, 2.3 \mu \mathrm{mol})$ were weighed out into a test tube with stir bar under nitrogen. To this was added toluene (Solvtek $\left.{ }^{\mathrm{TM}}, 600 \mu \mathrm{L}\right)$. The reaction was capped with a septum and heated at $80{ }^{\circ} \mathrm{C}$ for 7.5 hours under nitrogen. The crude reaction mixture was then cooled to room temperature and purified by flash column chromatography using ether and pentane (1:30). Product-containing fractions were collected and concentrated under reduced pressure to give $\mathbf{3 0}$ as an orange-red oil (34.8 $\mathrm{mg}, 65 \%$ yield).

$\mathbf{R}_{\mathbf{f}}: 0.37$ in pentane: ether, 49:1 (appears orange in visible light).

${ }^{1} \mathbf{H}$ NMR $\left(\mathrm{CDCl}_{3}, 400 \mathrm{MHz}\right) \delta: 7.40-7.31(\mathrm{~m}, 3 \mathrm{H}), 7.23-7.10(\mathrm{~m}, 7 \mathrm{H}), 2.33(\mathrm{t}, J=8.0 \mathrm{~Hz}, 2 \mathrm{H}), 2.23(\mathrm{t}, J$ $=8.4 \mathrm{~Hz}, 2 \mathrm{H}), 1.52(\mathrm{~m}, 2 \mathrm{H}), 1.15(\mathrm{~m}, 2 \mathrm{H}), 0.97(\mathrm{t}, J=7.6 \mathrm{~Hz}, 3 \mathrm{H}), 0.78(\mathrm{t}, J=7.2 \mathrm{~Hz} .3 \mathrm{H})$.

${ }^{13} \mathbf{C}$ NMR $\left(\mathrm{CDCl}_{3}, 100 \mathrm{MHz}\right) \delta: 202.6,156.4,154.1,134.6,131.0,129.7$ (2C), 128.6 (2C), 128.2, 128.1 (2C), 127.8 (2C), 126.9, 126.5, 124.7, 28.5, 25.9, 22.9, 21.6, 14.3, 14.1.

FTIR (thin film): 3396 (w), 3056 (m), 2960 (m), 2931 (m), 2870 (m), 1949 (w), 1881 (w), 1805 (w), 1707 (s), 1600 (m), 1573 (w), 1498 (m), 1463 (m), 1443 (m), 1357 (w), 1339 (w), 1307 (w), 1090 (m), $1071(\mathrm{~m}), 1027(\mathrm{w}), 970(\mathrm{w}), 914(\mathrm{w}), 756(\mathrm{~m}), 691(\mathrm{~m}) \mathrm{cm}^{-1}$.

HRMS Calculated for $\mathrm{C}_{23} \mathrm{H}_{24} \mathrm{NaO}^{+}(\mathrm{M}+\mathrm{Na})^{+}: 339.1725$, found: 339.1731 .

Elemental Analysis for $\mathrm{C}_{23} \mathrm{H}_{24} \mathrm{O}$ calc'd: $87.30 \%$ C, $7.64 \% \mathrm{H}$; found $87.34 \% \mathrm{C}, 7.49 \% \mathrm{H}$.<smiles>O=c1c(-c2ccccc2)c1-c1ccccc1I</smiles>

1

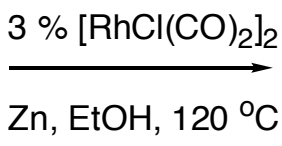

$69 \%$<smiles>O=C1C(c2ccccc2)=C(c2ccccc2)c2ccccc21</smiles>

32

2,3-Diphenyl-1-indenone ${ }^{9}(\mathbf{3 2})$ :

Diphenylcyclopropenone (1, $206 \mathrm{mg}, 1.0 \mathrm{mmol}), 1,2$-diiodobenzene $(165 \mathrm{mg}, 0.5 \mathrm{mmol})$, and activated zinc $^{10}(36 \mathrm{mg}, 0.55 \mathrm{mmol})$ were added to an oven dried borosilicate vial with stir bar. $\quad\left[\mathrm{RhCl}(\mathrm{CO})_{2}\right]_{2}(6.0$ $\mathrm{mg}, 1.5 \mu \mathrm{mol})$ and ethanol $(300 \mu \mathrm{L})$ were then added. The vial was flushed with argon, sealed tightly and heated at $120{ }^{\circ} \mathrm{C}$ for 3.5 hours before cooling back to room temperature. Ethanol was removed by blowing nitrogen into the vial. The reaction was purified by flash column chromatography eluting with ethyl acetate and pentane (1:20). Product-containing fractions were collected and concentrated under reduced pressure to give $\mathbf{3 2}$ as a red solid $(97 \mathrm{mg}, 69 \%)$. 32 prepared in this manner was identical $\left({ }^{1} \mathrm{H}\right.$ NMR) to commercially available 32 (Aldrich $\left.{ }^{\circledR}\right)$. 


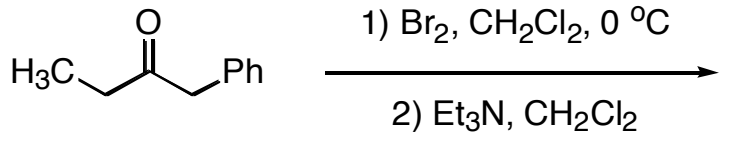

$45 \%$ Yield (2 steps)

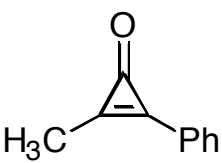

33

Methylphenylcyclopropenone ${ }^{11}(\mathbf{3 3})$ :

1-phenyl-2-butanone $(1.32 \mathrm{~g}, 8.9 \mathrm{mmol})$ and dichloromethane $(18 \mathrm{~mL}, 0.5 \mathrm{M})$ were added to an ovendried flask with a stir bar under nitrogen. The solution was cooled to $0{ }^{\circ} \mathrm{C}$ and bromine $(3.56 \mathrm{~g}, 22.3$ mmol) was added dropwise via syringe over 30 minutes. After addition was completed the reaction was stirred at room temperature for another 30 minutes. The reaction was poured into a separatory funnel containing $20 \mathrm{~mL}$ sat. aq. $\mathrm{Na}_{2} \mathrm{~S}_{2} \mathrm{O}_{3}$. The layers were separated and the aqueous layer was extracted with ether $(2 \times 15 \mathrm{~mL})$. The combined organics were washed with sat. aq. $\mathrm{NaHCO}_{3}(20 \mathrm{~mL})$ and brine $(20$ $\mathrm{mL}$ ), dried with $\mathrm{MgSO}_{4}$, filtered, and concentrated in vacuo. The yellowish oil was dissolved in dichloromethane $(18 \mathrm{~mL})$ and triethylamine $(9 \mathrm{~mL})$ and stirred at room temperature for 8 hours under nitrogen. The reaction was then diluted with ether $(100 \mathrm{~mL})$. The organics were washed with sat. aq. $\mathrm{CuSO}_{4}$ and brine (100 mL each), dried over sodium sulfate, filtered, and concentrated in vacuo. Further purification was accomplished by flash column chromatography eluting with ether. Product-containing fractions were concentrated to give light-brown solid $\mathbf{3 3}$ (572 mg, 45\%).

m. p. $66-68^{\circ} \mathrm{C}$.

$\mathbf{R}_{\mathbf{f}}: 0.25$ in ether (uv).

${ }^{1} \mathbf{H}$ NMR $\left(\mathrm{CDCl}_{3}, 400 \mathrm{MHz}\right)$ 8: 7.78-7.72 (m, 2H), 7.58-7.48 (m, 3H), 2.47 (s, 3H).

${ }^{13}$ C NMR $\left(\mathrm{CDCl}_{3}, 100 \mathrm{MHz}\right) \delta: 155.9,154.0,151.2,131.9,130.3$ (2C), 128.6 (2C), 123.1, 10.9.

FTIR (thin film): 3467 (b), 3059 (w), 2960 (w), 2922 (w), 1856 (s), 1626 (s), 1448 (m), 1333 (w), 1021 (w), $770(\mathrm{~m}), 692(\mathrm{~m}), 678(\mathrm{w}) \mathrm{cm}^{-1}$.

HRMS for $\mathrm{C}_{10} \mathrm{H}_{8} \mathrm{NaO}^{+}(\mathrm{M}+\mathrm{Na})^{+}$cac'd: 167.0473 , found: 167.0467.<smiles>Cc1c([PH2+])c1=O</smiles>

33
Cyclopentadienone 3:

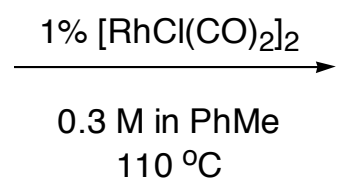

$57 \%$<smiles>CC1=C(c2ccccc2)C(=O)C(c2ccccc2)=C1c1ccccc1</smiles>

3

Methylphenylcyclopropenone (33, $220 \mu \mathrm{mol}, 31.7 \mathrm{mg})$, diphenylacetylene $(35.7 \mathrm{mg}, 200 \mu \mathrm{mol}$ and $\left[\mathrm{RhCl}(\mathrm{CO})_{2}\right]_{2}(1 \mathrm{mg}, 2.6 \mu \mathrm{mol})$ were weighed out into a test tube with stir bar under nitrogen. To this was added toluene (Solvtek $\left.{ }^{\mathrm{TM}}, 540 \mu \mathrm{L}\right)$. The tube was capped with a septum and heated at $110{ }^{\circ} \mathrm{C}$ for 17.5 hours under nitrogen. The resulting solution was purified by flash column chromatography eluting with $100 \mathrm{~mL}$ petroleum ether followed by $150 \mathrm{~mL} \mathrm{5 \%}$ diethyl ether/petroleum ether. Product-containing fractions were concentrated under reduced pressure to leave cyclopentadienone $\mathbf{3}$ as a deep purple solid (36.6 mg, 57\%). 3 prepared in this manner is identical ( $\left.{ }^{1} \mathrm{H} \mathrm{NMR}\right)$ to 3 prepared in the manner described on page $\mathrm{S} 2$. 

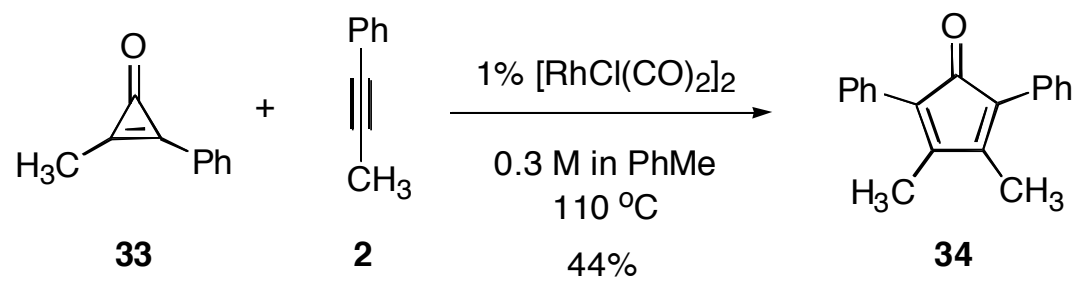

Cyclopentadienone 34:

Methylphenylcyclopropenone (33, $31.7 \mathrm{mg}, 220 \mu \mathrm{mol})$, 1-phenyl-1-propyne (2, $23.2 \mathrm{mg}, 200 \mu \mathrm{mol})$, and $\left[\mathrm{RhCl}(\mathrm{CO})_{2}\right]_{2}(1.0 \mathrm{mg}, 2.6 \mu \mathrm{mol})$ were weighed out into a test tube with stir bar under nitrogen. To this was added toluene (Solvtek ${ }^{\mathrm{TM}}, 440 \mu \mathrm{L}$ ). The tube was capped with a septum and heated at $110{ }^{\circ} \mathrm{C}$ for 7.5 hours under nitrogen. The crude reaction mixture was then cooled to room temperature and purified by flash column chromatography eluting with ethyl acetate and pentane (1:24). Product fractions were collected and concentrated under reduced pressure to give $\mathbf{3 4}$ as a red solid ( $23 \mathrm{mg}$, $44 \%$ yield).

mp: $131-133^{\circ} \mathrm{C}$.

$\mathbf{R}_{\mathbf{f}}: 0.50$ in pentane: ether, 19:1 (appears red-orange in visible light).

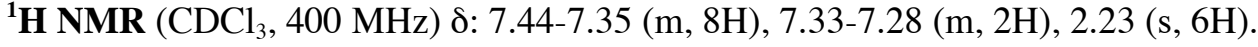

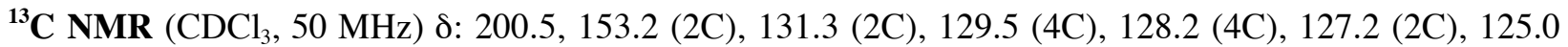
(2C), $13.0(2 \mathrm{C})$.

FTIR (thin film): 3398 (w), 3047 (w), 2951 (w), 2920 (w), 2360 (w), 2342 (w), 1956 (w), 1892 (w), 1855 (w), 1703 (s), 1442 (m), 1377 (m), 1297 (m), 1275 (w), 1081 (w), 977 (w), $922(\mathrm{w}), 846(\mathrm{w}), 795(\mathrm{~m})$, $740(\mathrm{~m}), 701(\mathrm{~s}) \mathrm{cm}^{-1}$.

HRMS for $\mathrm{C}_{19} \mathrm{H}_{16} \mathrm{O}$ calc'd: $260.1201 \mathrm{~g} / \mathrm{mol}$, found $260.1192 \mathrm{~g} / \mathrm{mol}$.

Elemental Analysis for $\mathrm{C}_{19} \mathrm{H}_{16} \mathrm{O}$ calc'd: $87.66 \%$ C, $6.19 \% \mathrm{H}$; found $87.34 \% \mathrm{C}, 6.07 \% \mathrm{H}$.
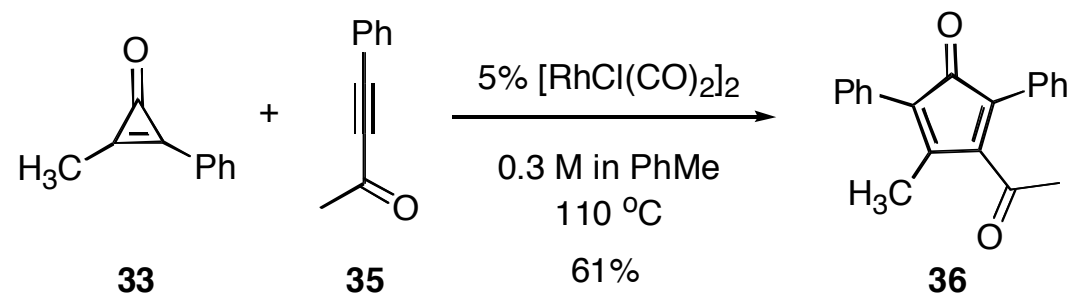

Cyclopentadienone 36:

Methylphenylcyclopropenone (33, $19.9 \mathrm{mg}, 138 \mu \mathrm{mol})$, 4-phenyl-but-3-yn-2-one (35, $15.3 \mathrm{mg}, 106$ $\mu \mathrm{mol})$, and $\left[\mathrm{RhCl}(\mathrm{CO})_{2}\right]_{2}(2.1 \mathrm{mg}, 5.4 \mu \mathrm{mol})$ were weighed out into a test tube with stir bar under nitrogen. To this was added toluene (Solvtek ${ }^{\mathrm{TM}}, 600 \mu \mathrm{L}$ ). The tube was capped with a septum and heated at $110{ }^{\circ} \mathrm{C}$ for 5 hours under nitrogen. The crude reaction mixture was then cooled to room temperature and purified by flash column chromatography eluting with ethyl acetate and pentane (1:24). Product fractions were collected and concentrated under reduced pressure to give $\mathbf{3 6}$ as a red oil (18.6 $\mathrm{mg}, 61 \%$ yield).

$\mathbf{R}_{\mathbf{f}}: 0.33$ in 9:1, pentane: ether (appears red in visible light).

${ }^{1} \mathbf{H}$ NMR $\left(\mathrm{CDCl}_{3}, 400 \mathrm{MHz}\right)$ 8: 7.47-7.31 (m, 10H), 2.24 (s, 3H), 2.23 (s, 3H). 
${ }^{13} \mathbf{C}$ NMR $\left(\mathrm{CDCl}_{3}, 100 \mathrm{MHz}\right) \delta: 202.1,199.8,154.2,149.6,130.5,129.54$ (2C), 129.52 (2C), 129.3, 128.6 (2C), 128.3 (2C), 128.1, 127.7, 125.9, 77.2, 30.9, 14.3.

FTIR (thin film): 3055 (w), 2920 (w), 1717 (s), 1684 (s), 1492 (m), 1443 (m), 1380 (m), 1358 (m), 1298 (w), $1175(\mathrm{~m}), 1112(\mathrm{~m}), 1077$ (w), 1025 (w), 974 (w), 933 (w), 796 (m), 755 (w), $739(\mathrm{~m}), 697(\mathrm{~m}) \mathrm{cm}^{-1}$.

HRMS for $\mathrm{C}_{20} \mathrm{H}_{16} \mathrm{O}_{2}$ calc'd: $288.1150 \mathrm{~g} / \mathrm{mol}$, found $288.1144 \mathrm{~g} / \mathrm{mol}$. 
III. Selected ${ }^{1} \mathrm{H}$ NMR Spectra for New Compounds.

Cyclopentadienone 3:
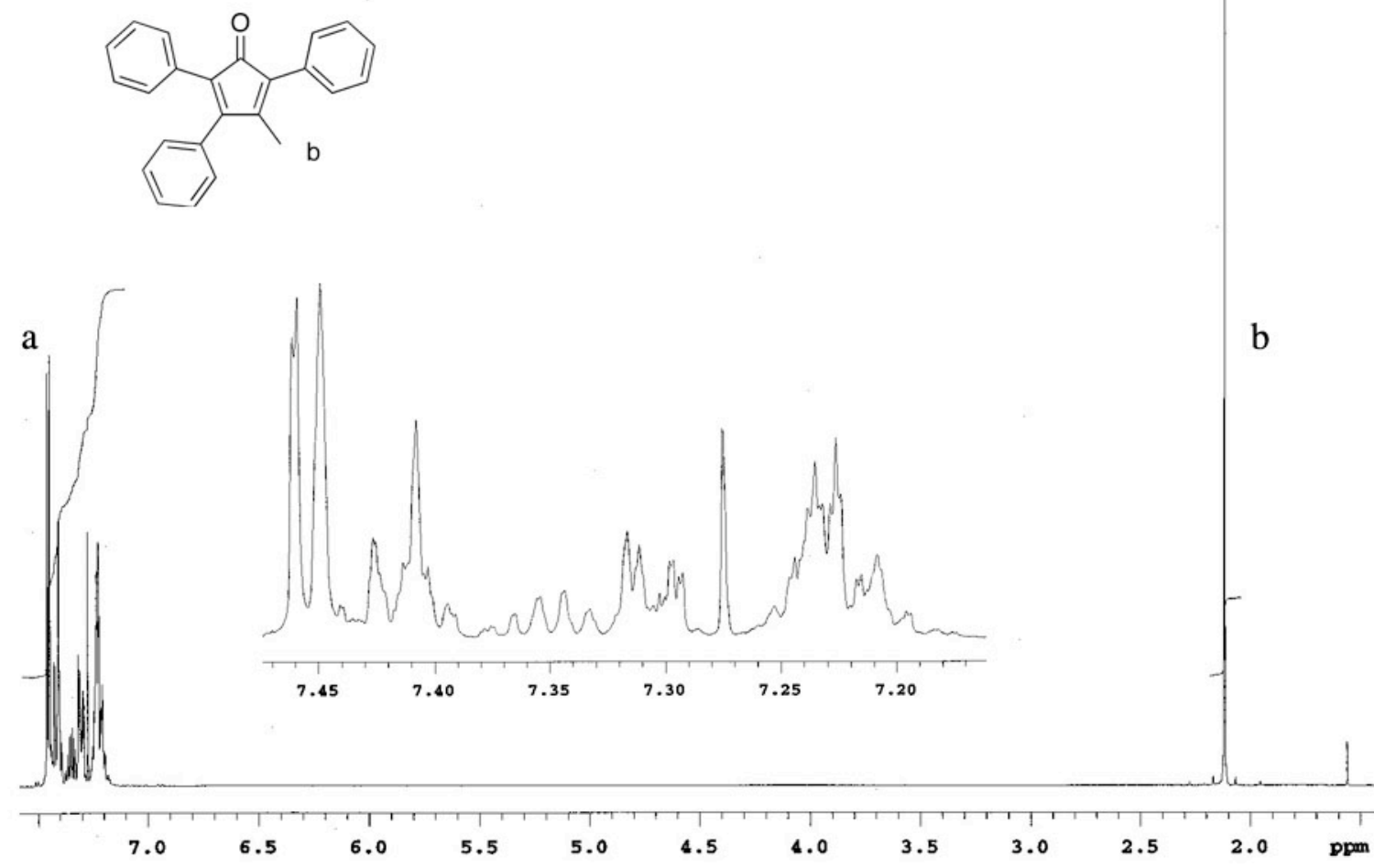

Cyclopentadienone 4:
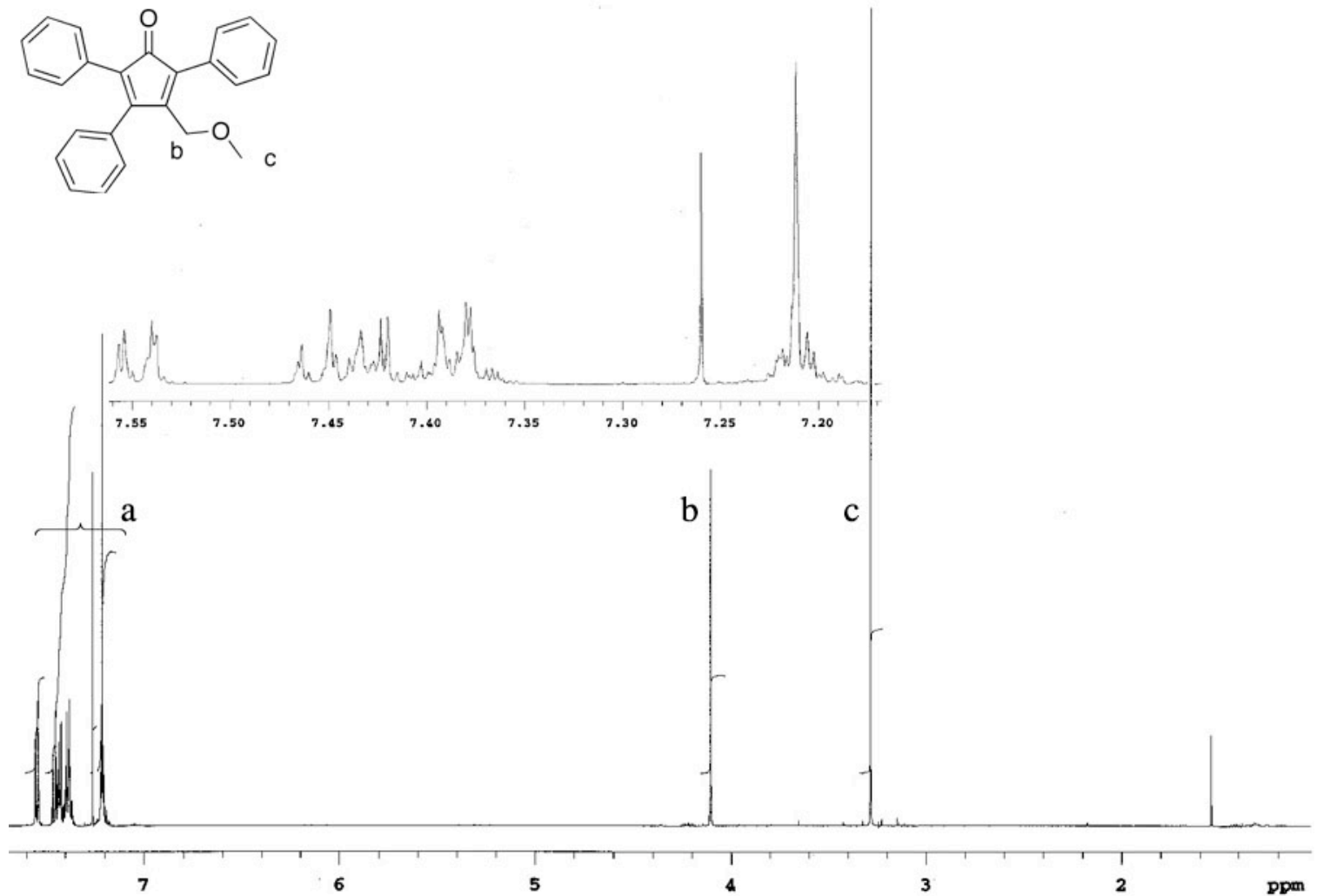
Cyclopentadienone 5:

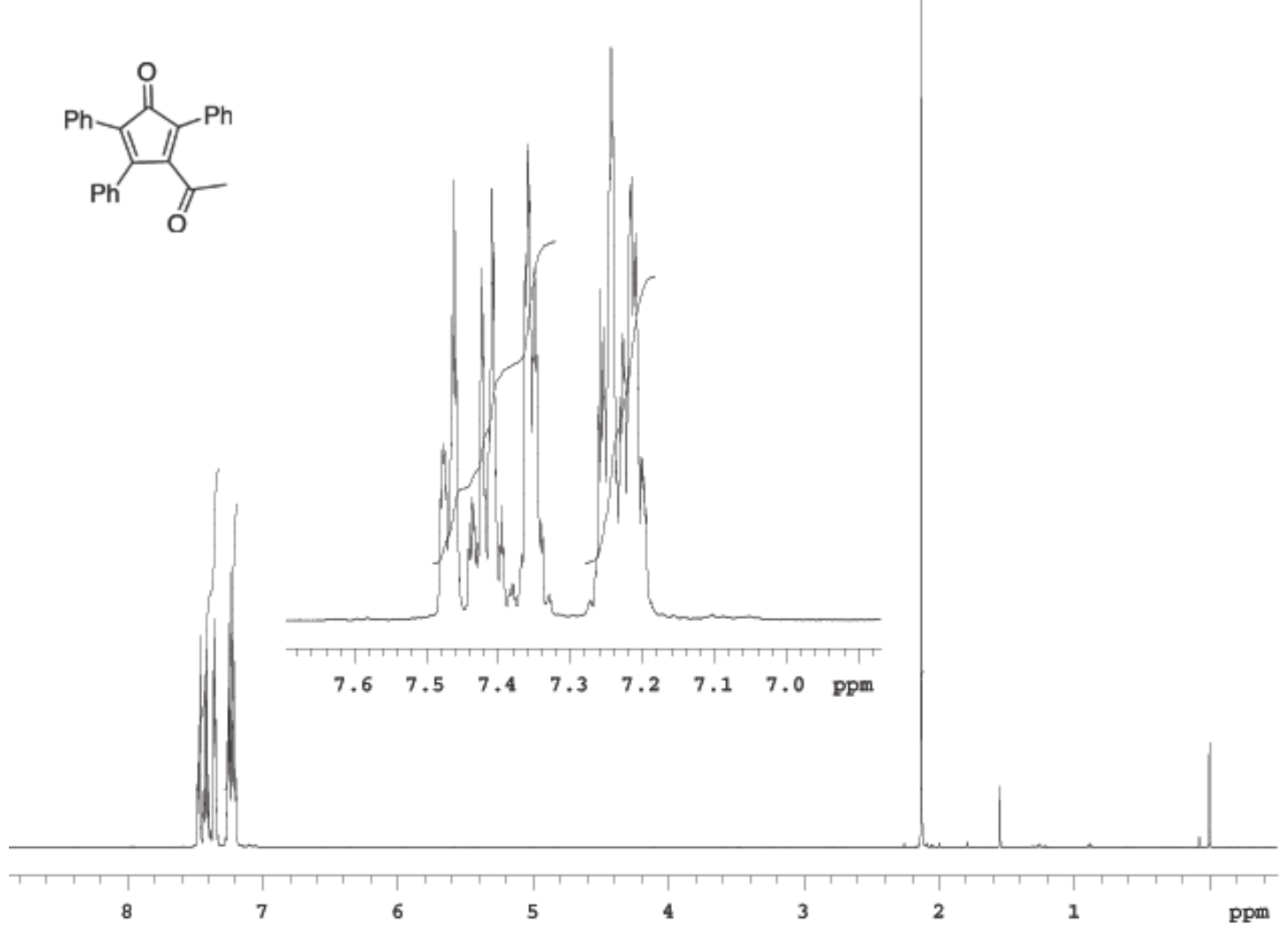

Cyclopentadienone 6:

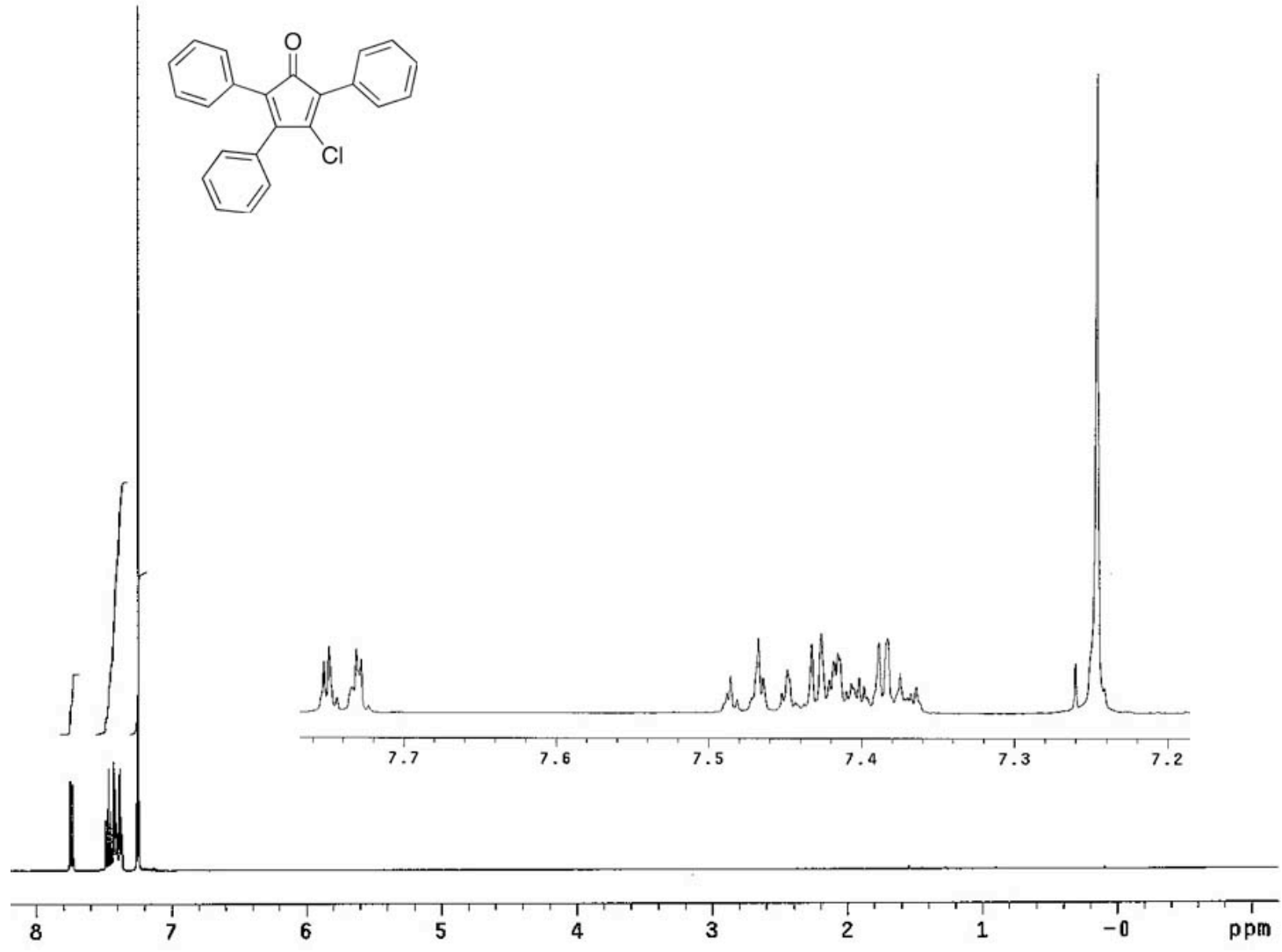


Cyclopentadienone 7:

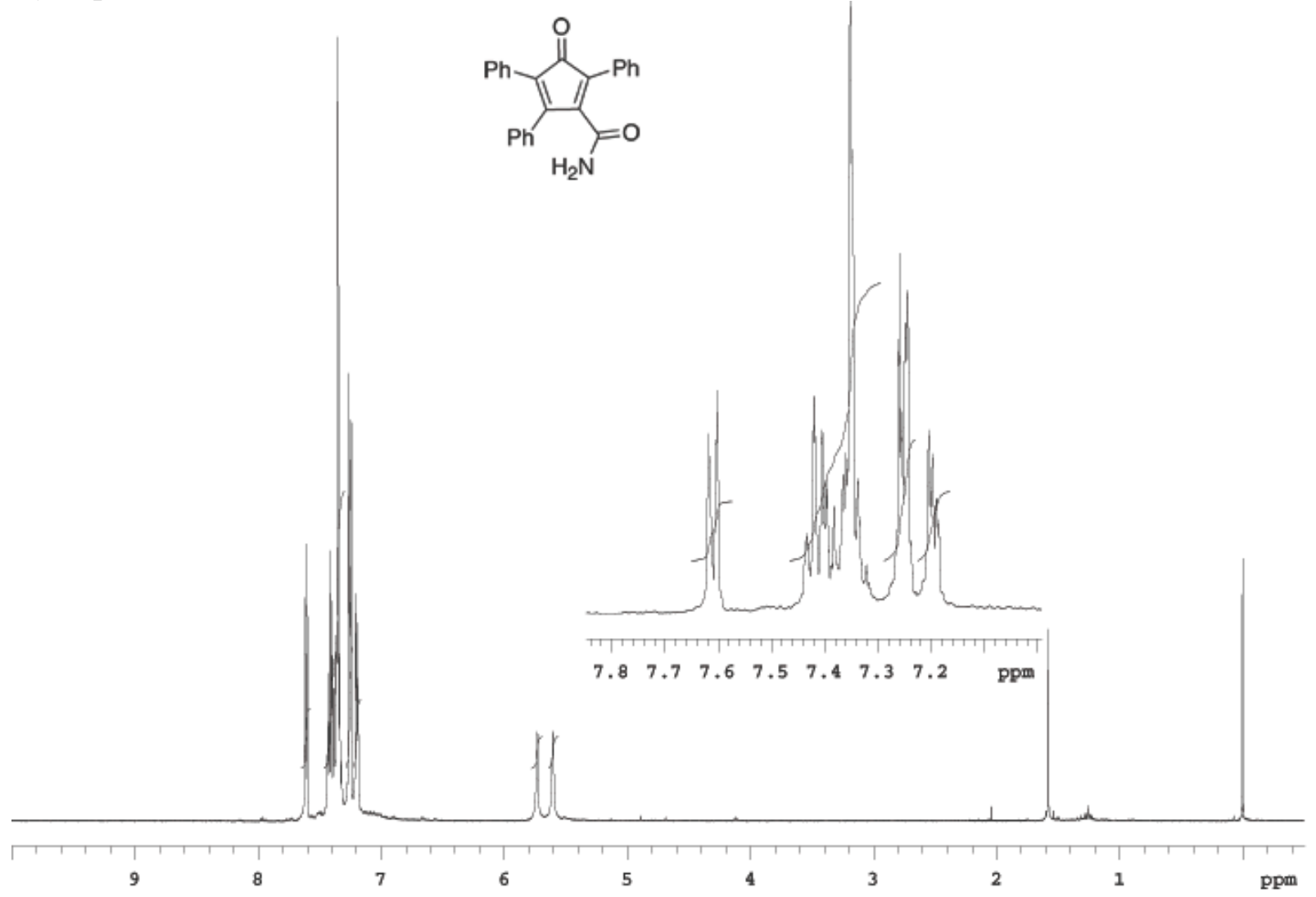

Cyclopentadienone 8:

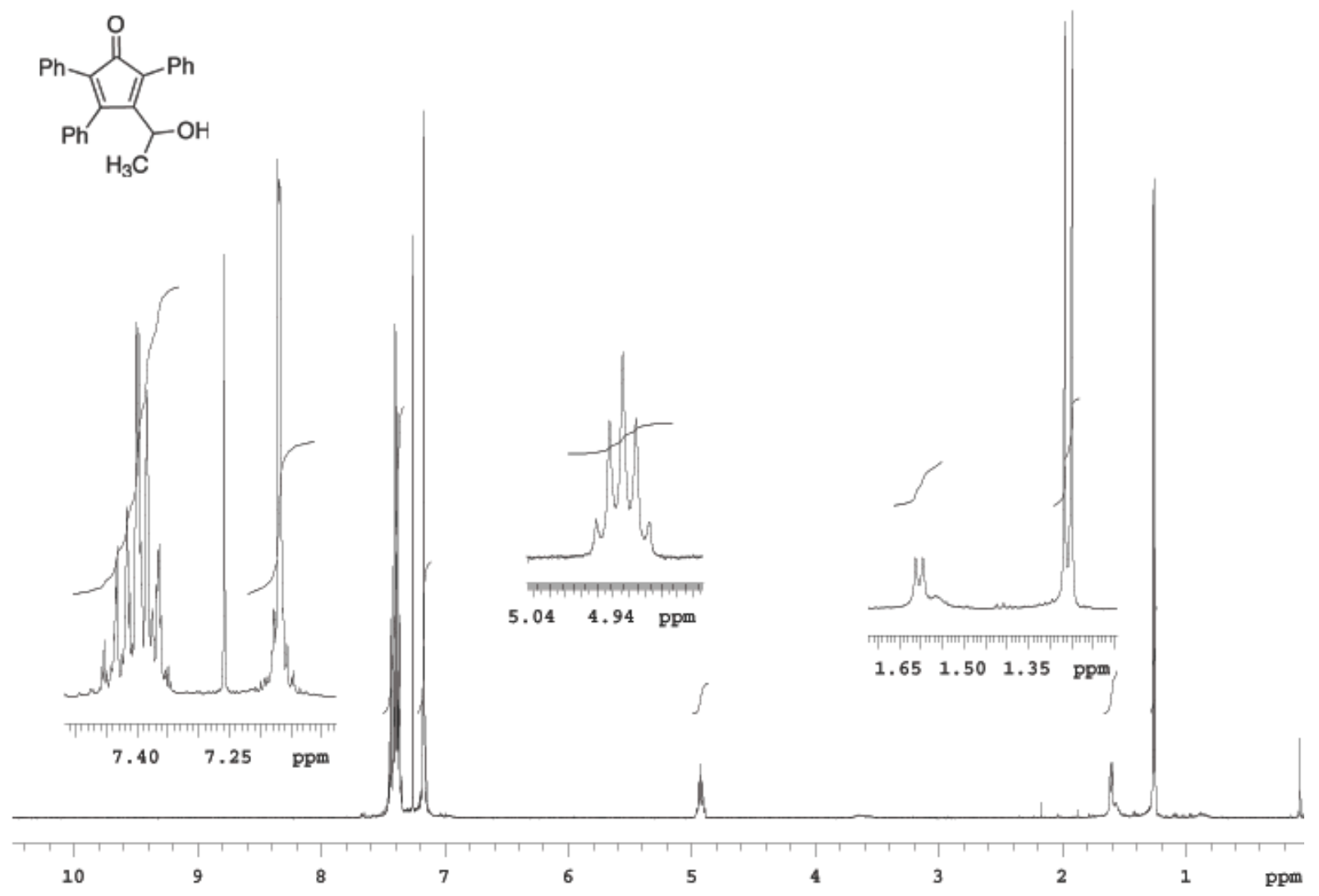


Cyclopentadienone 9:

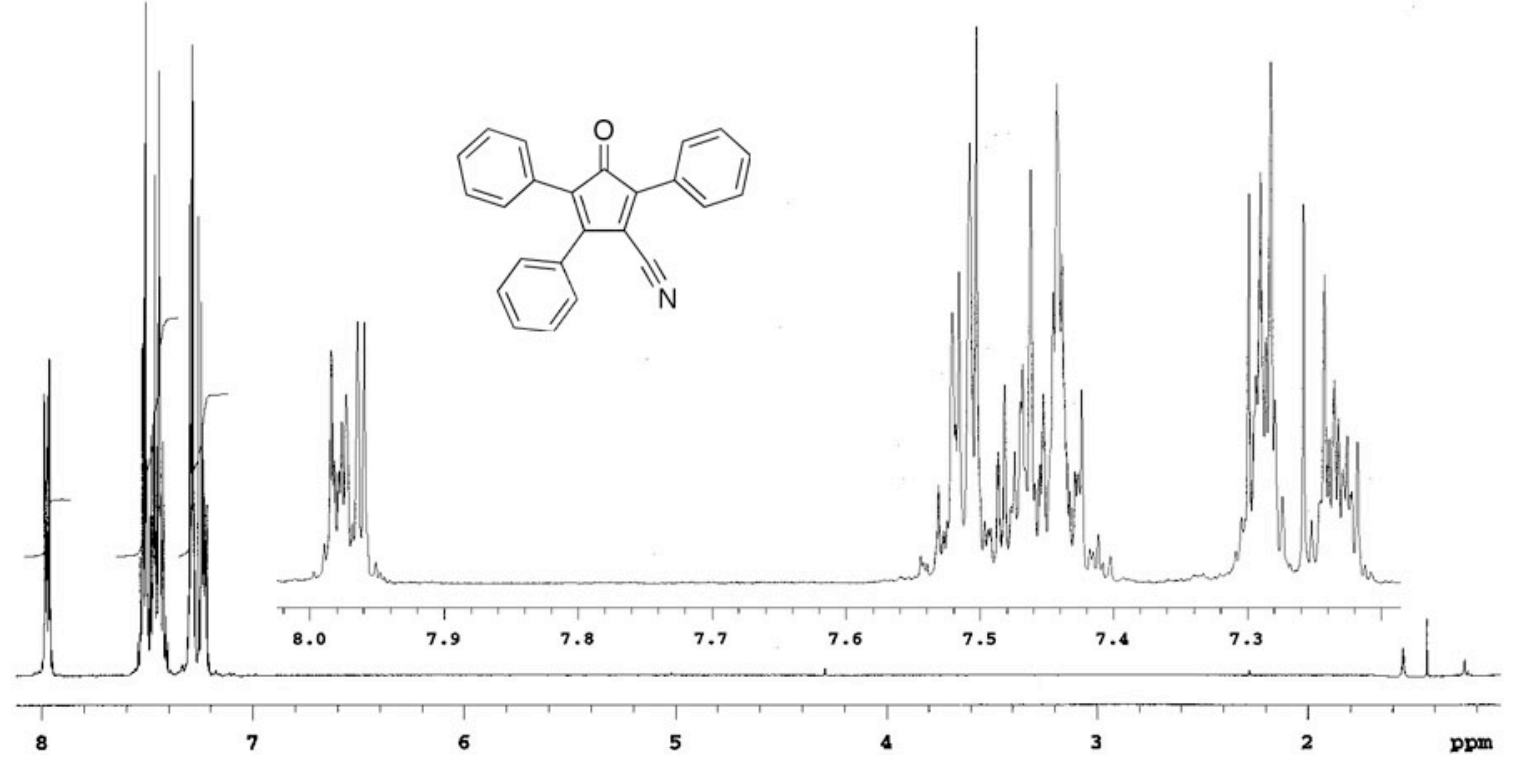

Cyclopentadienone 10:

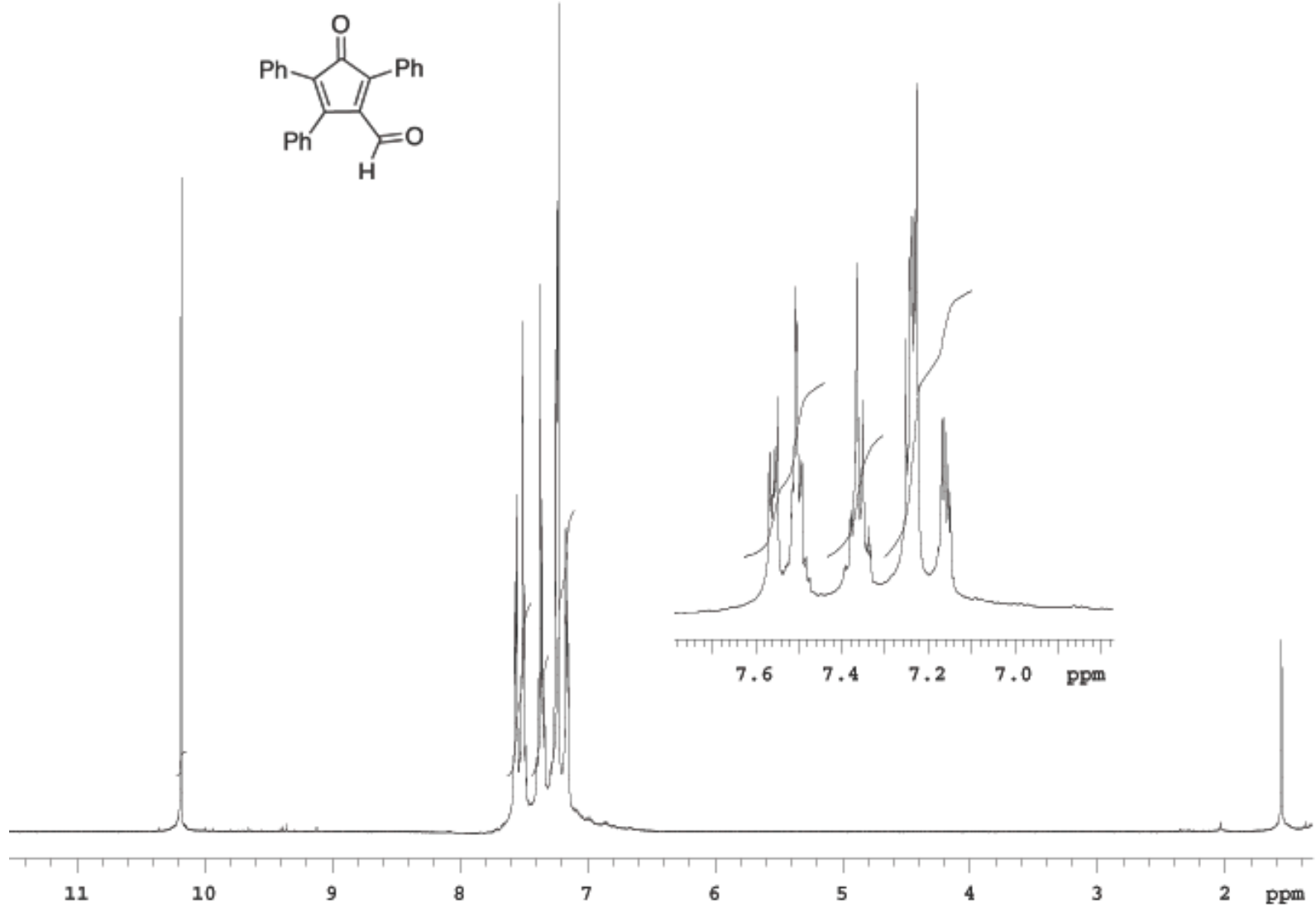




\section{Cyclopentadienone 11:}

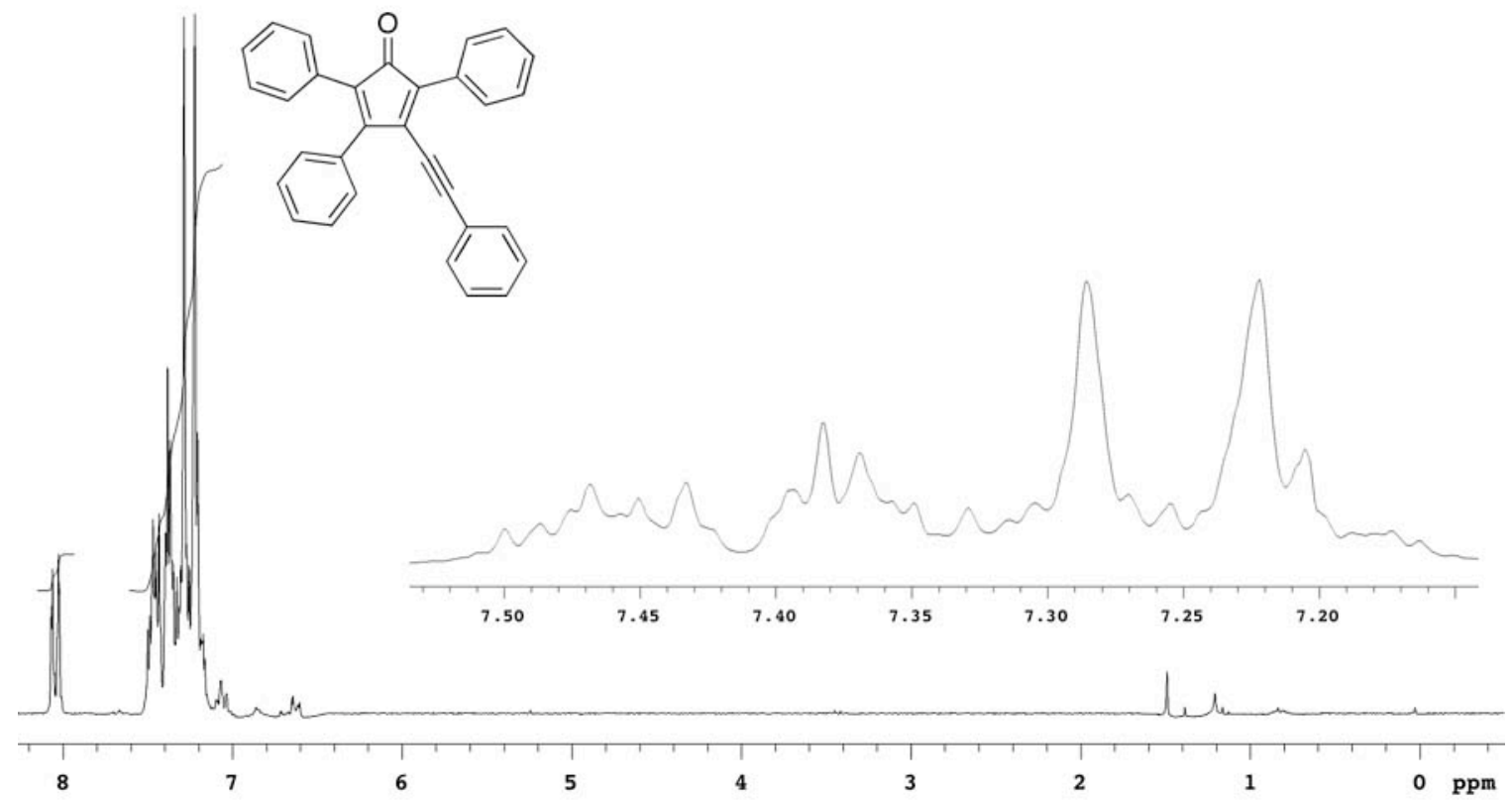

Cyclopentadienone 11a:

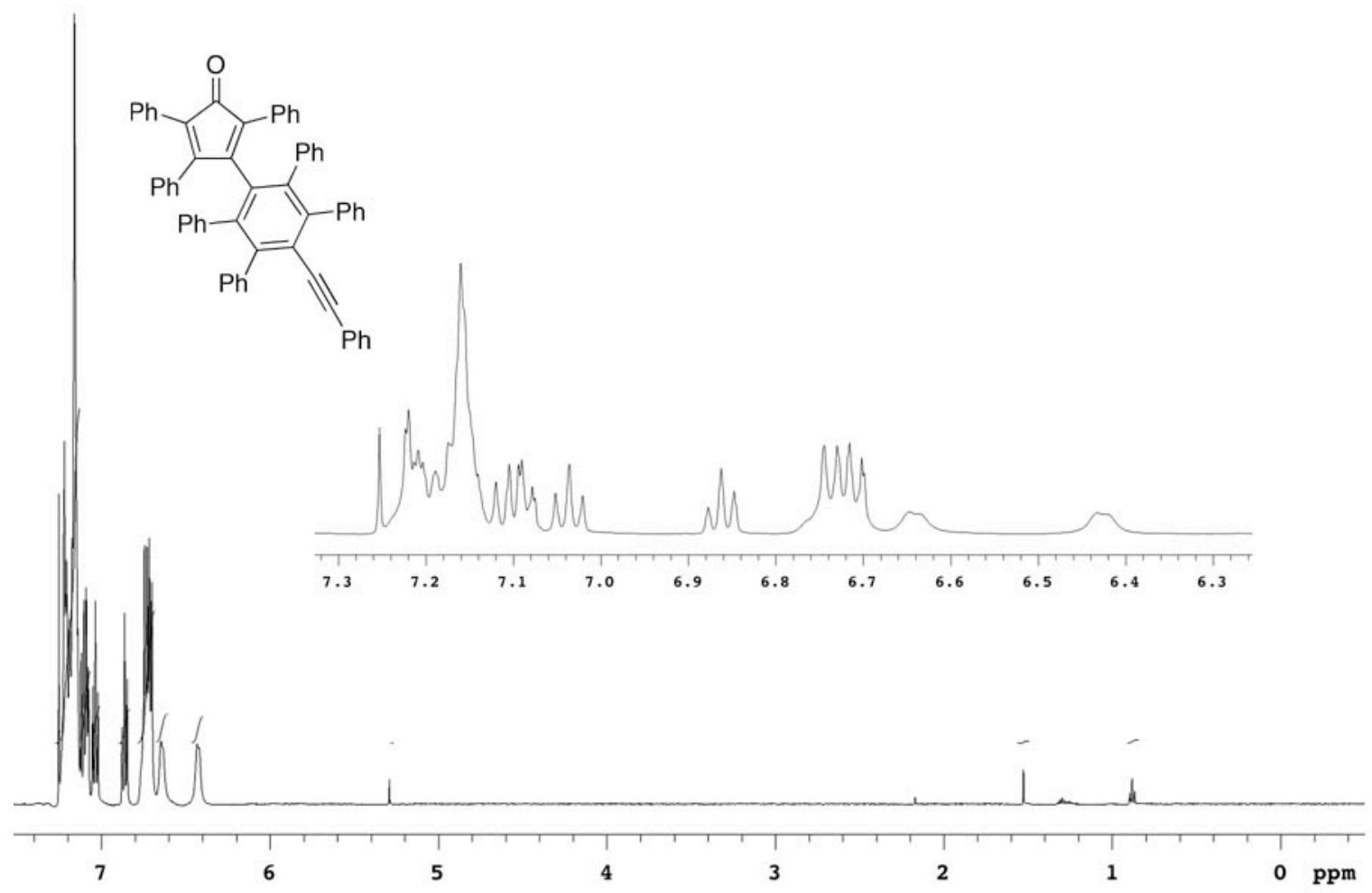


Cyclopentadienone 12:

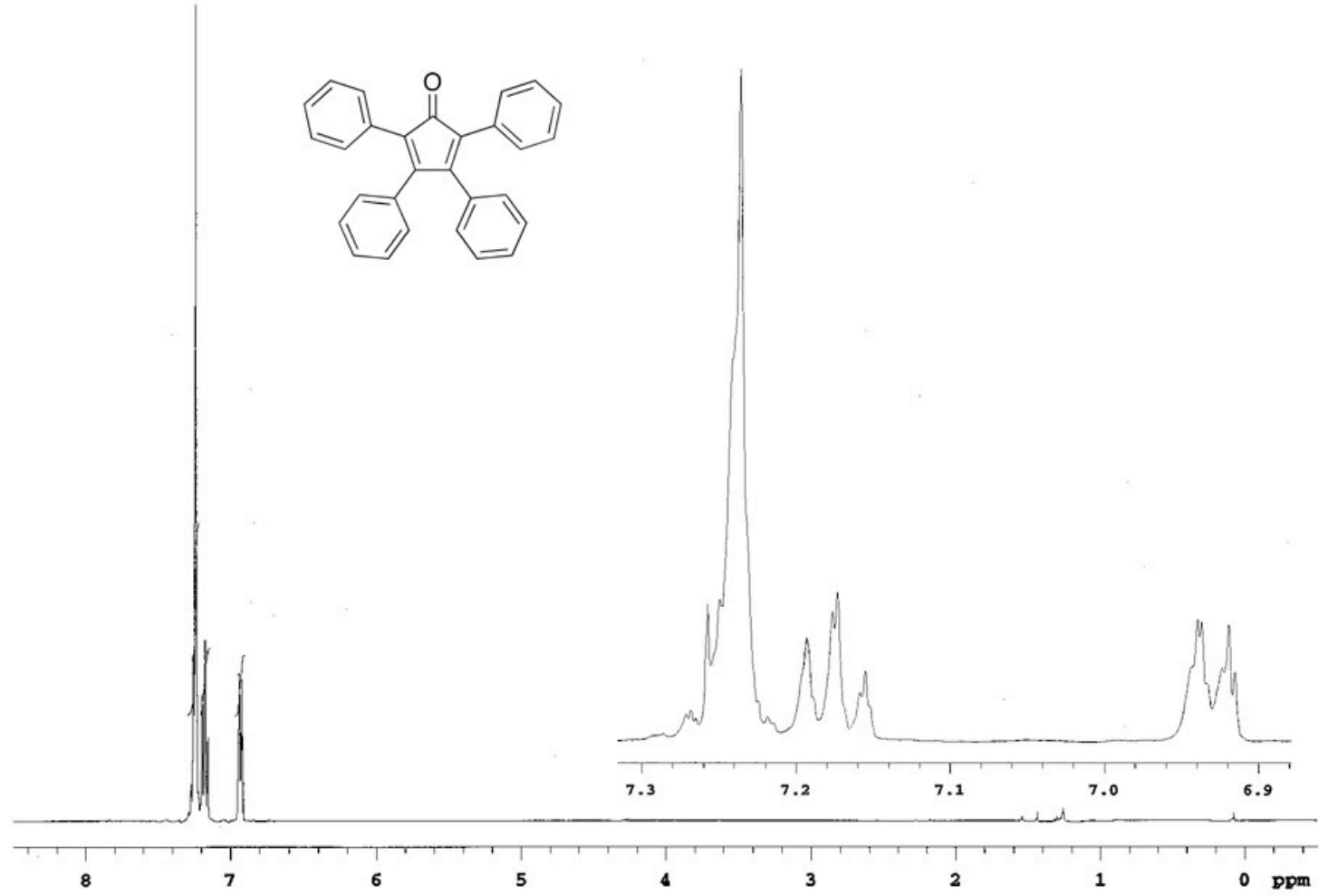

Cyclopentadienone 14:

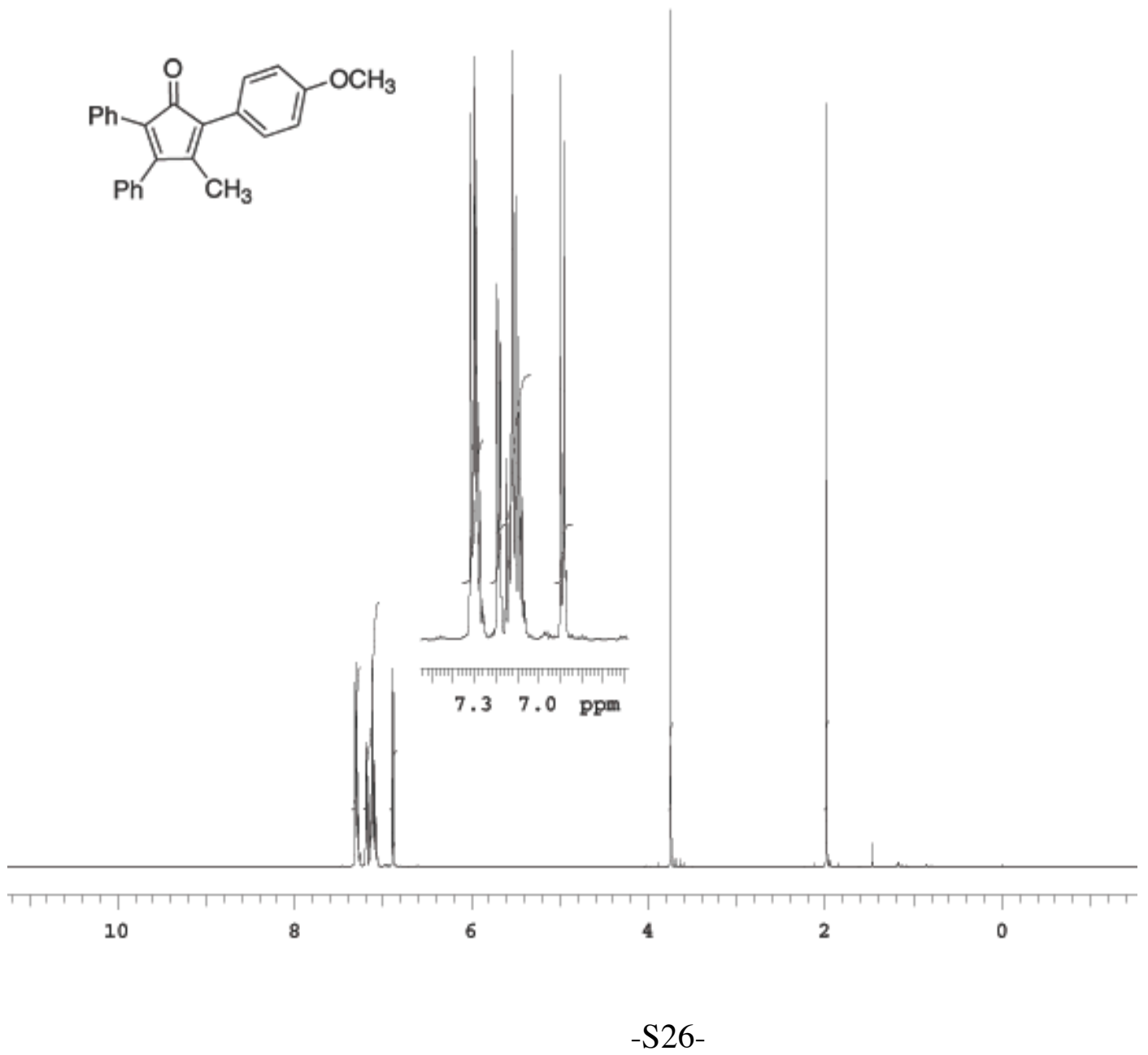


Cyclopentadienone 16:

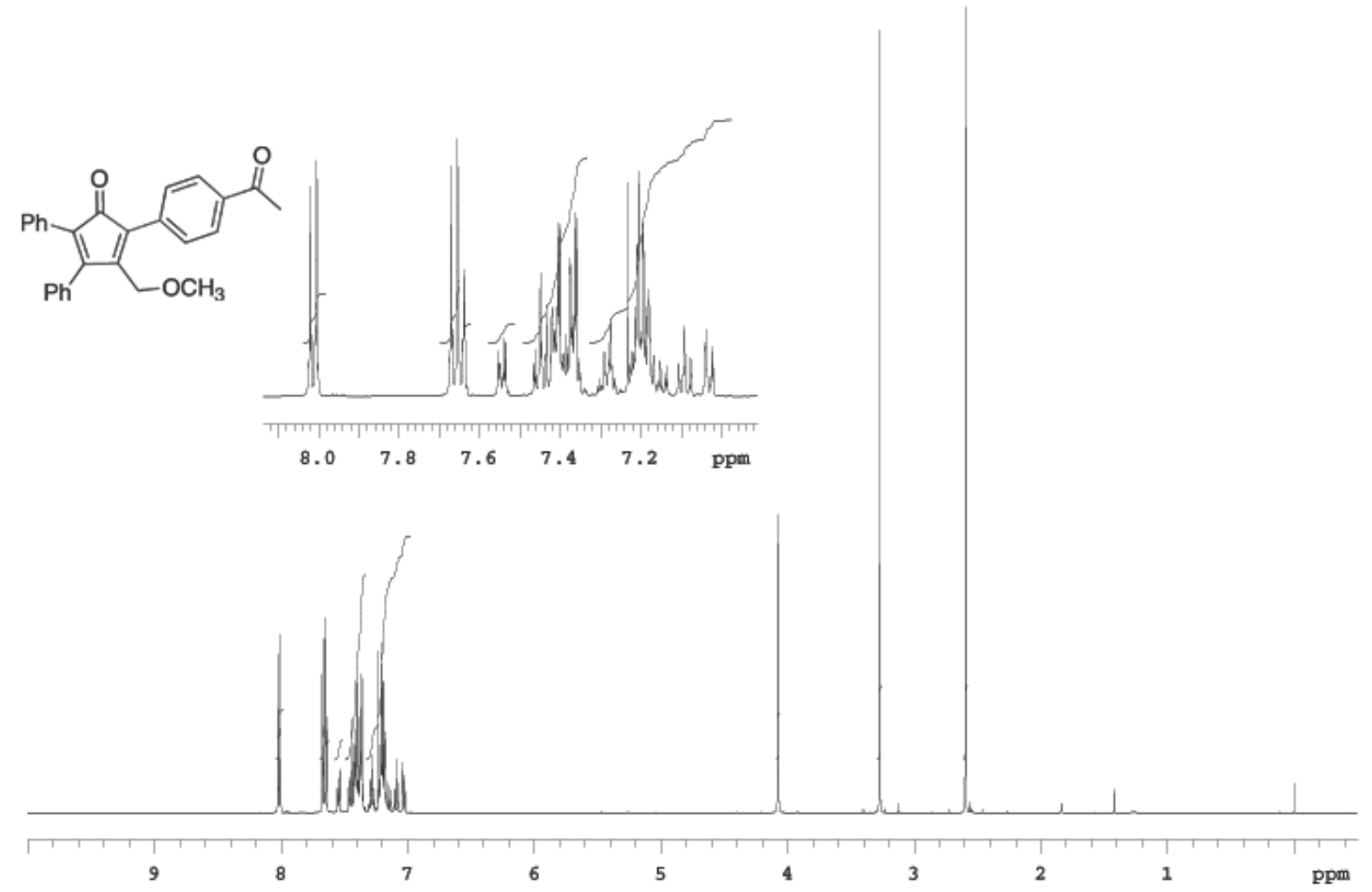

Cyclopentadienone 18:

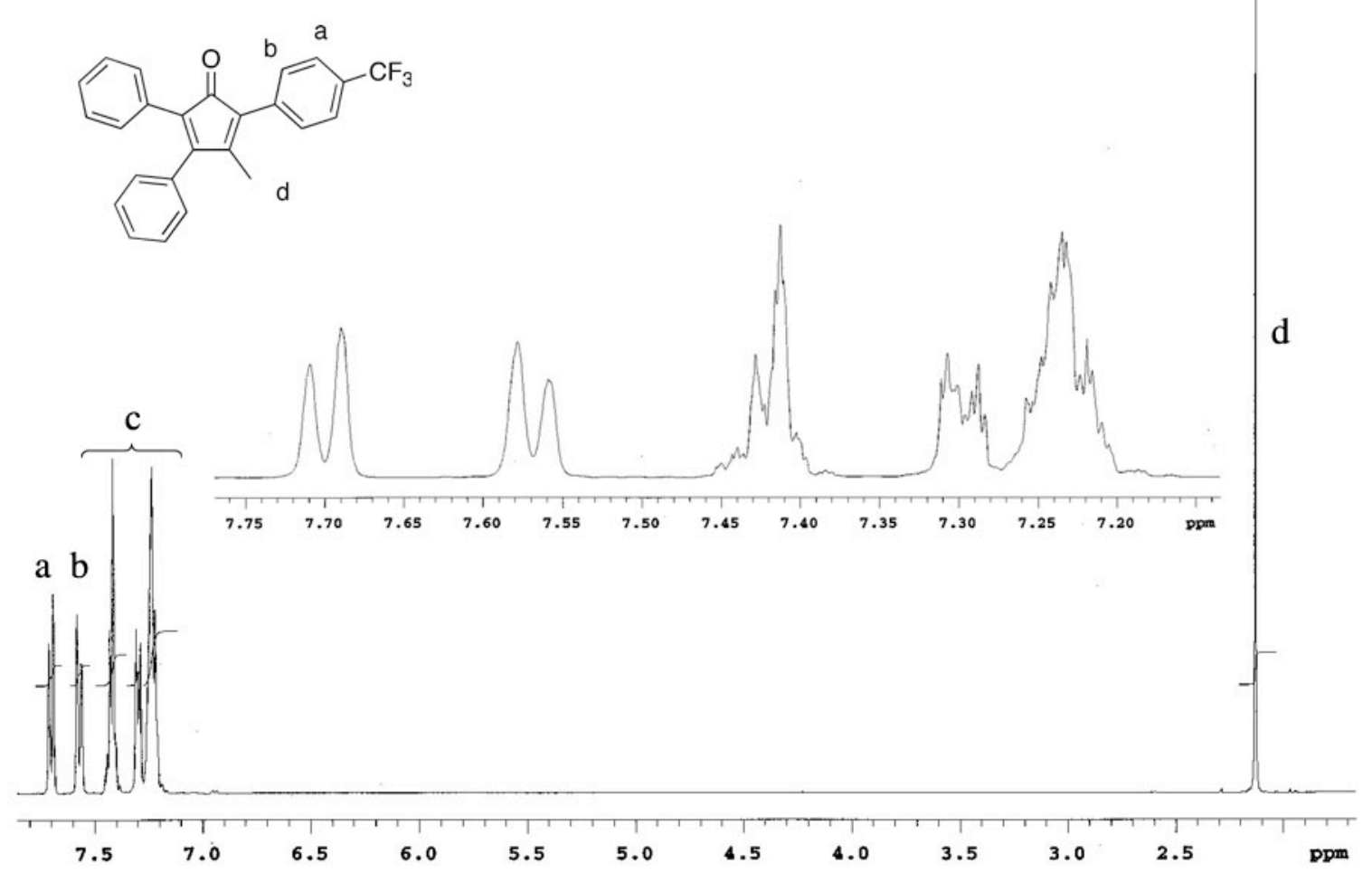


Cyclopentadienone 20:

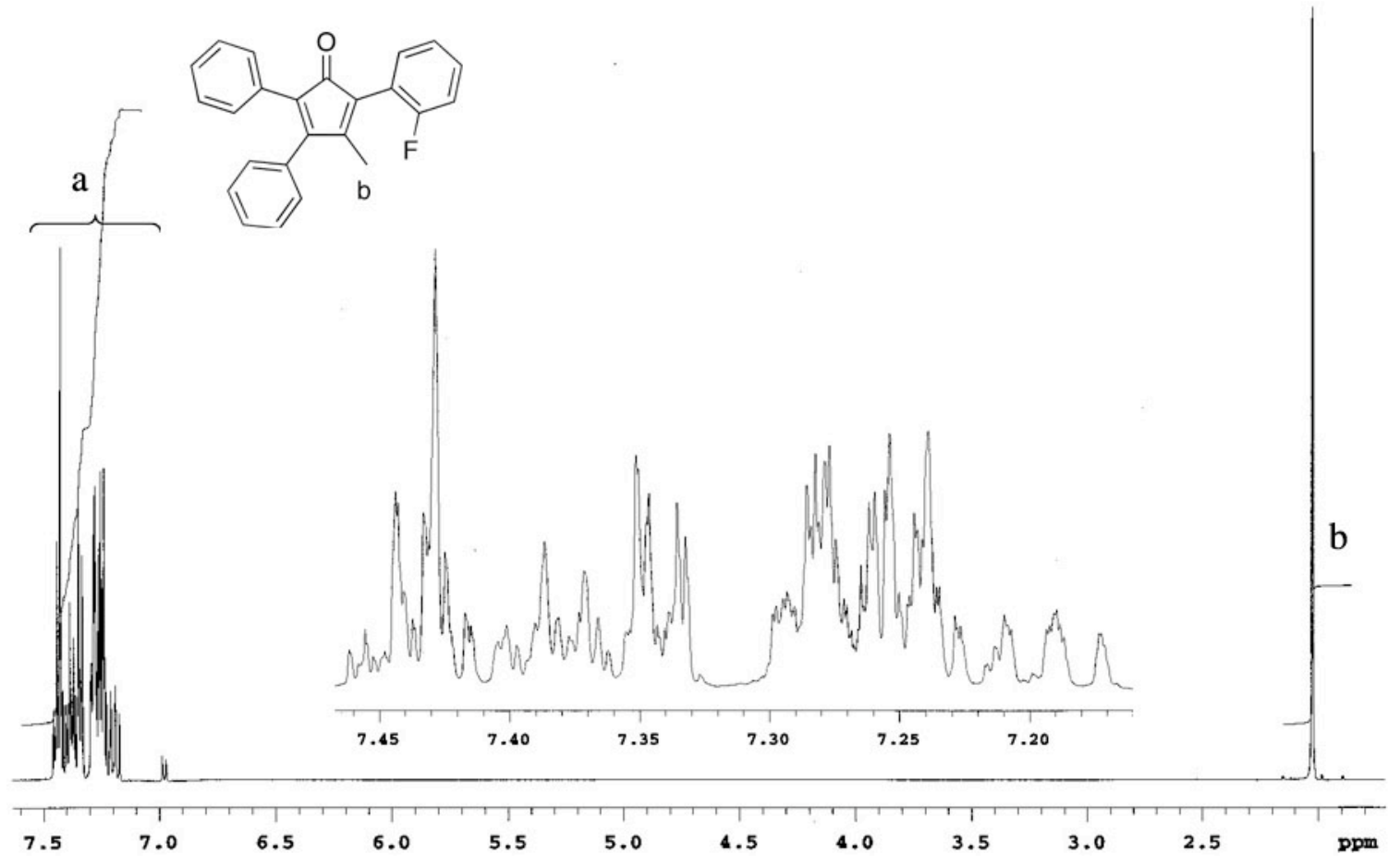

Cyclopentadienone 22:

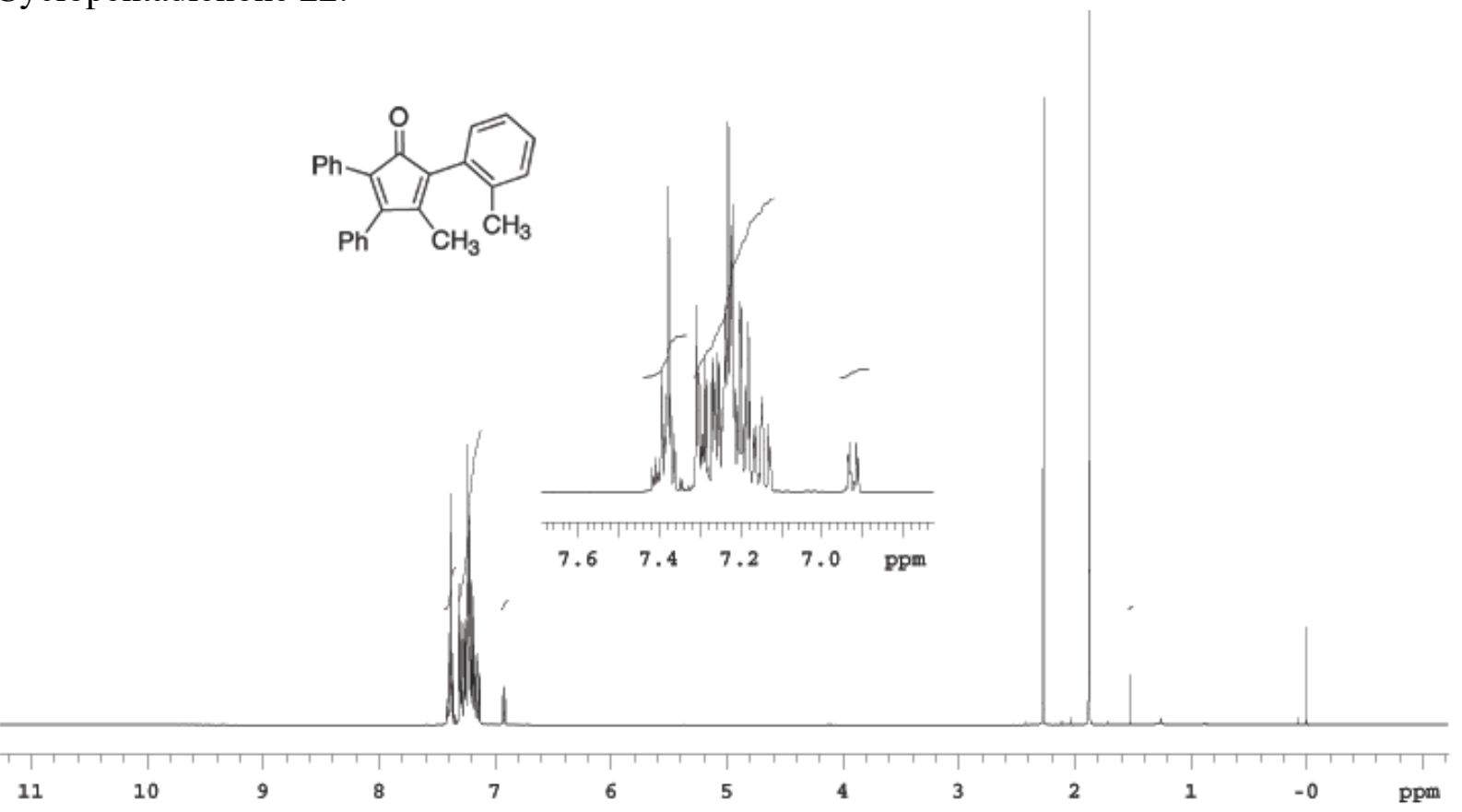


Cyclopentadienone 24:
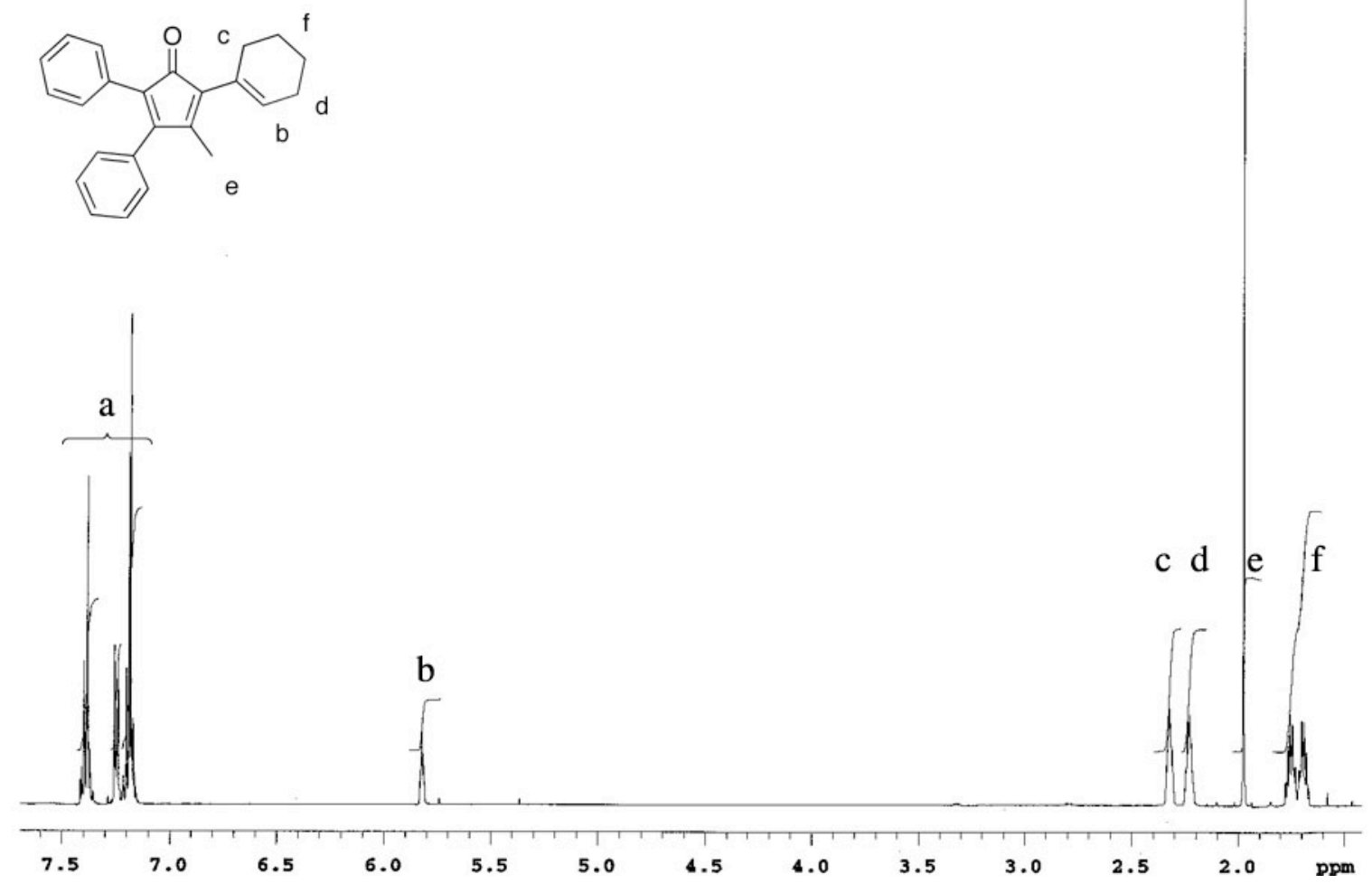

Cyclopentadienone 26:
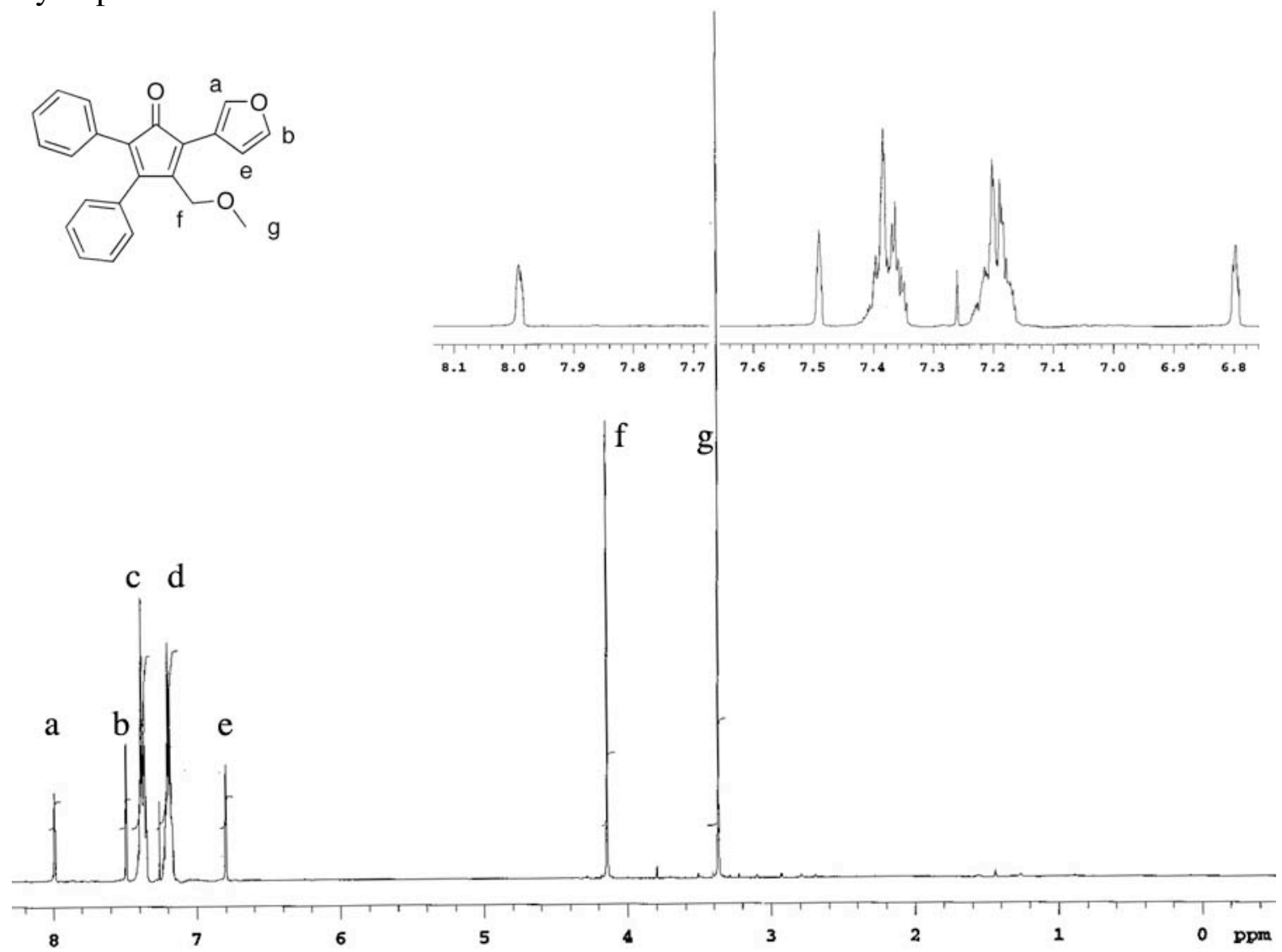
Cyclopentadienone 28:

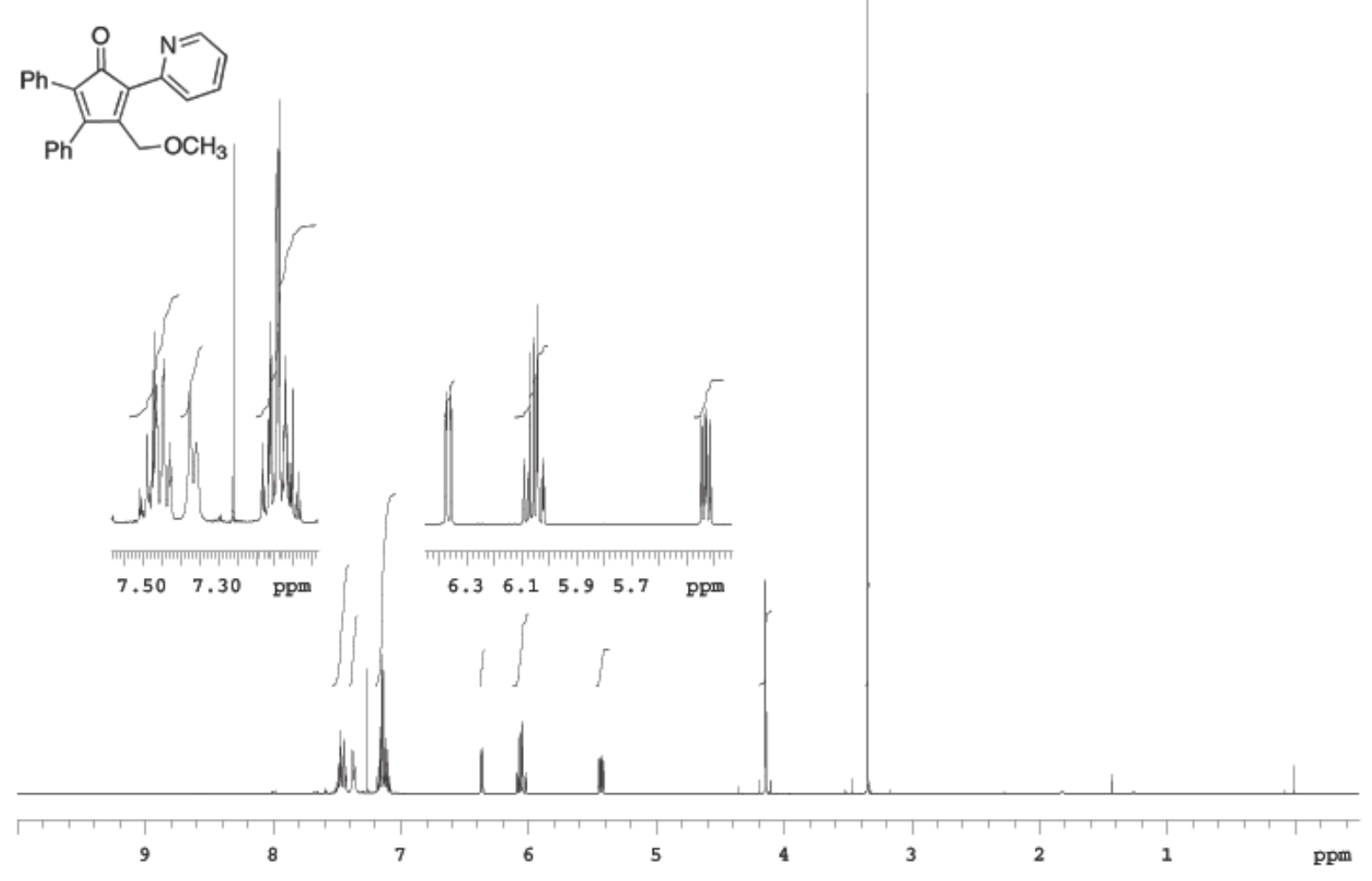

\section{Cyclopentadienone 30:}

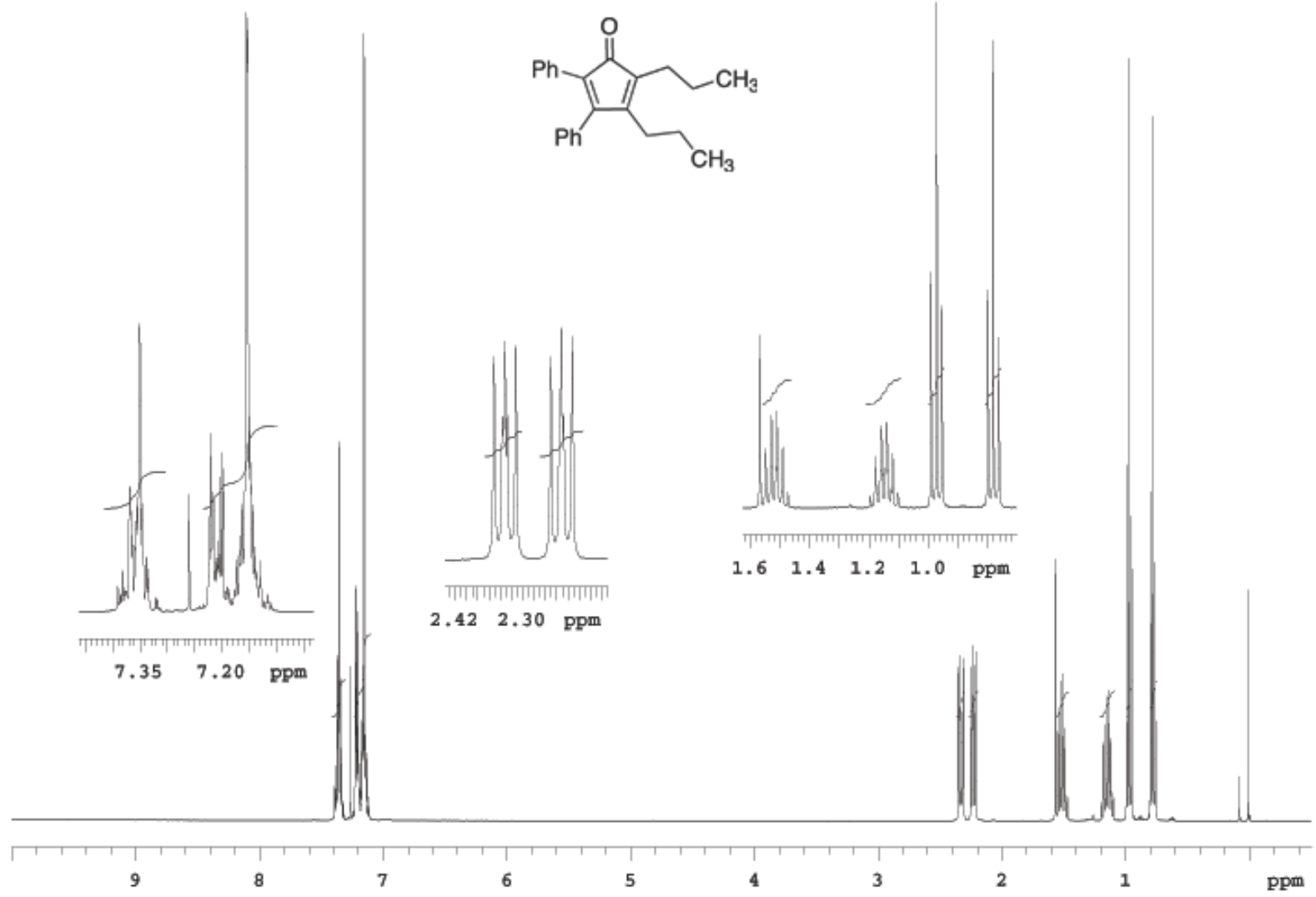


Methylphenylcyclopropenone (33):
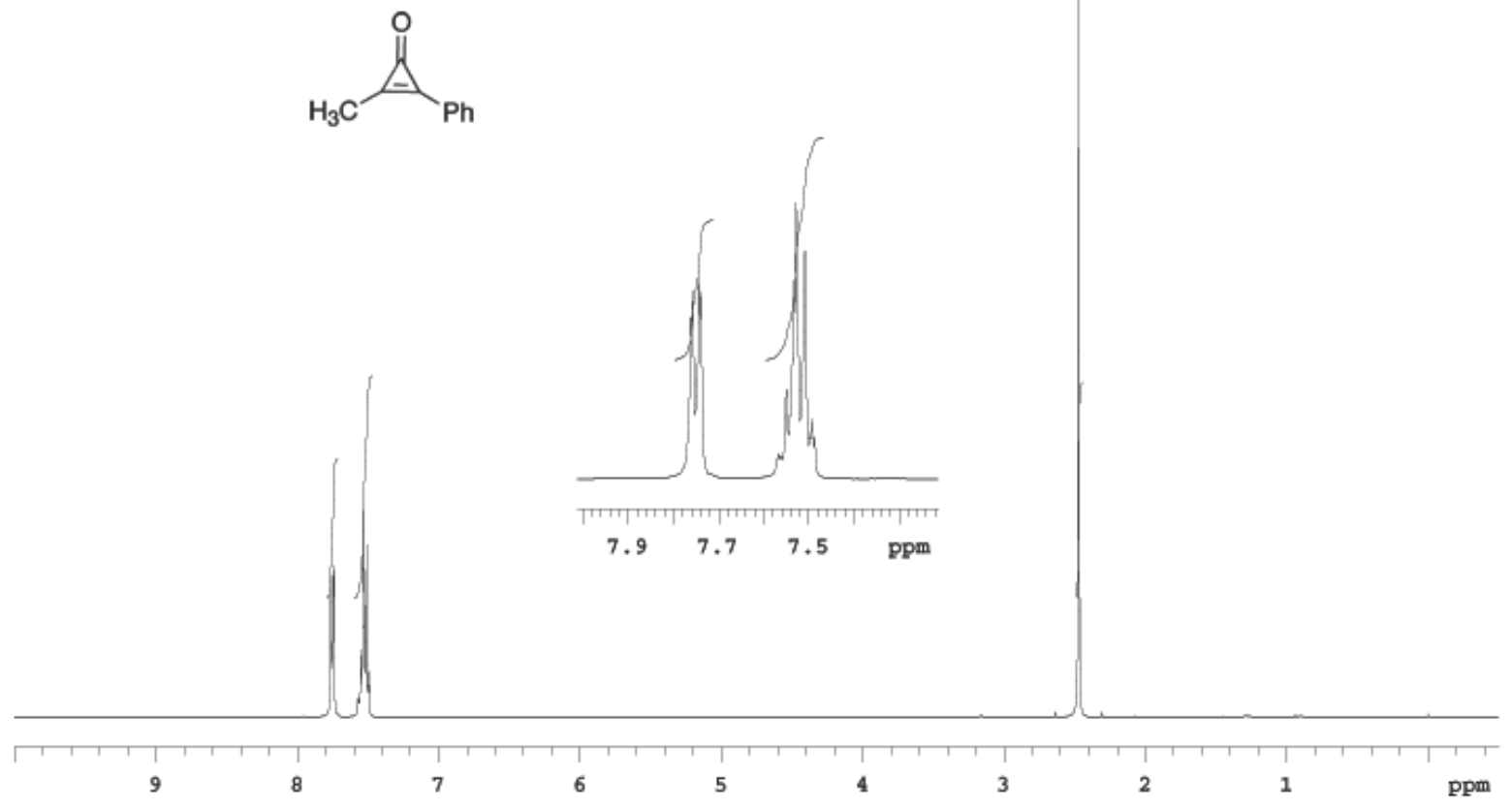

Cyclopentadienone 34:

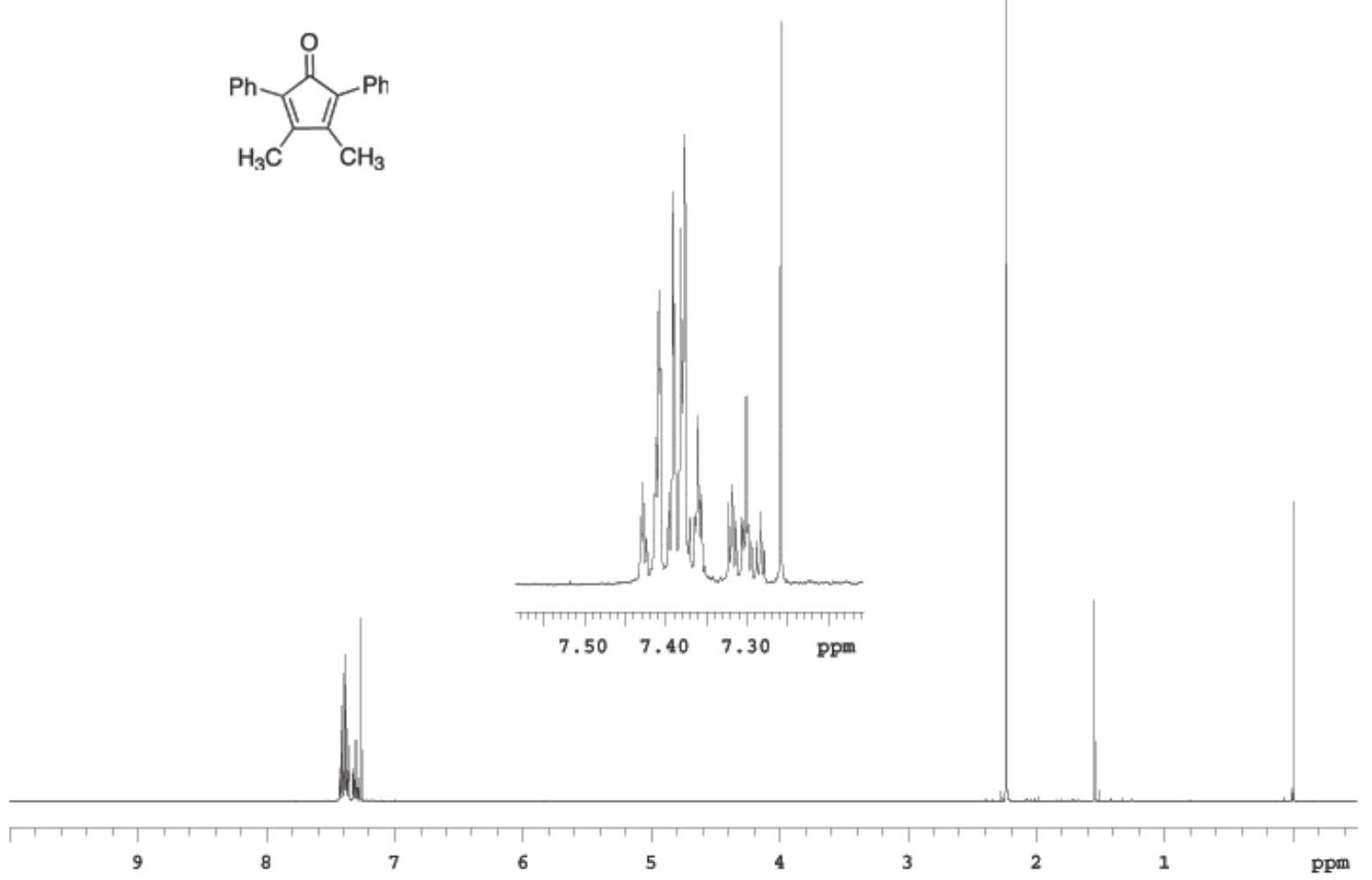


Cyclopentadienone 36:

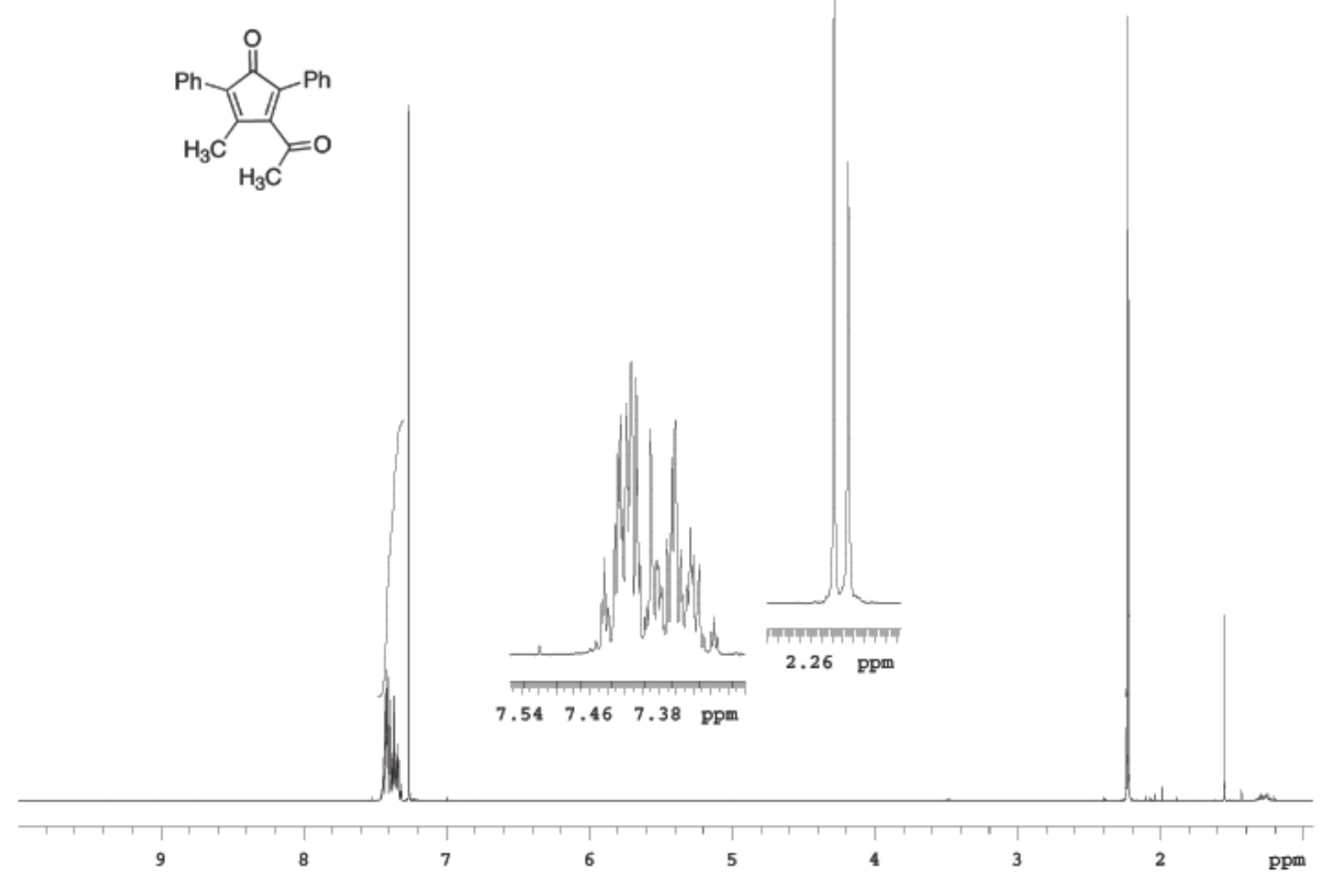

\section{X-Ray Crystallographic Data for Compound 3.}

A purple plate crystal of $\mathrm{C}_{24} \mathrm{H}_{18} \mathrm{O}$ having approximate dimensions of $0.25 \times 0.17 \times 0.01 \mathrm{~mm}$ was mounted on a quartz fiber using Paratone $\mathrm{N}$ hydrocarbon oil. All measurements were made on a Bruker-Siemens SMART CCD area detector with monochromatic radiation of wavelength $0.71073 \AA$.

Cell constants and an orientation matrix for data collection, obtained from a least-squares refinement using the measured positions of 1166 centered reflections with $I>10 \sigma(I)$ in the range $2.38^{\circ}<\theta<23.29^{\circ}$, corresponded to a primitive monoclinic cell with dimensions:

$$
\begin{array}{lll}
\mathrm{a}=8.983(2) \AA & \alpha=90^{\circ} & \mathrm{V}=1693.6(7) \AA^{3} \\
\mathrm{~b}=16.458(4) \AA & \beta=108.031(4)^{\circ} & \\
\mathrm{c}=12.047(3) \AA & \gamma=90^{\circ} &
\end{array}
$$

For $Z=4$ and $F . W .=322.38$, the calculated density is $1.264 \mathrm{~g} / \mathrm{cm}^{3}$. Based on a statistical analysis of intensity distribution, and the successful solution and refinement of the structure, the space group was determined to be $\mathrm{P} 2_{1} / \mathrm{c}$. The data were collected at a temperature of $133 \mathrm{~K}$. Frames corresponding to an arbitrary hemisphere of data were collected using $\omega$ scans of $0.3^{\circ}$ counted for a total of 40 seconds per frame.

Data were integrated by the program SAINT with box parameters of $1.6 \times 1.6 \times 1.0^{\circ}$ to a maximum $\theta$ value of $24.96^{\circ}$. The data were corrected for Lorentz and polarization effects. The linear absorption coefficient, $\mu$, for $0.71073 \AA$ radiation is $0.076 \mathrm{~mm}^{-1}$. Data were analyzed for agreement and possible absorption using SADABS. ${ }^{3}$ A semi-empirical absorption correction based on 7.07 reflections with $I>$ $5 \sigma(I)$ was applied that resulted in normalized transmission factors ranging from 0.39 to 1 . Of the 7336 reflections that were collected, 2746 were unique $\left(R_{\text {int }}=0.0821\right)$; equivalent reflections were merged. A 
correction for secondary extinction $^{3 a}$ was applied having a refined coefficient of $0.012(3)$. No decay correction was deemed necessary.

The structure was solved by direct methods (SIR-97) and expanded using Fourier techniques. All nonhydrogen atoms were refined with anisotropic thermal parameters. Hydrogen atoms were located on the difference Fourier map, but were positioned using the HFIX command (idealized positions via a riding refinement). A single torsional parameter about the $\mathrm{C}-\mathrm{C}$ bond was refined in the case of the methyl group. The final cycle of full-matrix least-squares refinement was based on 2746 reflections (all data) and 228 variable parameters and converged (largest parameter shift was 0.000 times its esd) with conventional unweighted and weighted agreement factors of $R_{1}=\Sigma\left\|F_{o}|-| F_{c}\right\| / \Sigma\left|F_{o}\right|=0.0577$ for 1687 data with $F_{o}>$ $4 \sigma\left(F_{o}\right)$, and $w R_{2}=\left[\left(\Sigma w\left(\left|F_{o}\right|^{2}-\left|F_{c}\right|^{2}\right)^{2} / \Sigma w \mid F_{o}{ }^{2}\right)\right]^{1 / 2}=0.1716$.

The standard deviation of an observation of unit weight $(S)^{7}$ was 0.940 . Sheldrick weights ${ }^{6}$ were used; where applicable, weights were refined to convergence. The maximum and minimum peaks on the final difference Fourier map corresponded to 0.29 and $-0.23 \mathrm{e}^{-} / \AA^{3}$, respectively.

Table 2. Crystal Data.

\begin{tabular}{ll}
\hline Empirical Formula & $\mathrm{C}_{24} \mathrm{H}_{18} \mathrm{O}$ \\
Formula Weight & 322.38 \\
Crystal Color, Habit & Purple, plate \\
Crystal Dimensions & $0.25 \times 0.17 \times 0.01 \mathrm{~mm}$ \\
Crystal System & Monoclinic \\
Lattice Type & Primitive \\
Lattice Parameters & $\mathrm{a}=8.983(2) \AA ; \mathrm{b}=16.458(4) \AA ; \mathrm{c}=12.047(3) \AA$ \\
& $\alpha=90^{\circ} ; \beta=108.031(4)^{\circ} ; \gamma=90^{\circ}$ \\
& $\mathrm{V}=1693.6(7) \AA^{3}$ \\
Space Group & $\mathrm{P} 2 / \mathrm{c}$ \\
$\mathrm{Z}$ value & 4 \\
$\mathrm{D}_{\text {calc }}$ & $1.264 \mathrm{~g} / \mathrm{cm}^{3}$ \\
F000 & 680 \\
$\mu(0.71073 \AA$ radiation $)$ & $0.08 \mathrm{~cm}^{-1}$ \\
\hline
\end{tabular}

Table 3. Intensity Measurements.

\begin{tabular}{ll}
\hline Diffractometer & Siemens SMART APEX CCD \\
Radiation & $\lambda=0.71073 \AA$ A, graphite monochromated \\
Temperature & $133 \mathrm{~K}$ \\
Scan Type & $\omega\left(0.3^{\circ} /\right.$ frame $)$ \\
Exposure Time & 40 seconds per frame \\
$\theta_{\max }$ & $24.96^{\circ}$ \\
No. of Reflections Measured & Total: 7336 ; Unique: $2746\left(R_{\text {int }}=0.0821\right)$ \\
Corrections & Lorentz-polarization \\
& Absorption: $T_{\max }=1, T_{\min }=0.39$ \\
\hline
\end{tabular}

Table 4. Structure Solution and Refinement.

\begin{tabular}{ll}
\hline Structure Solution & Direct (SIR-97) \\
Refinement & Full-matrix least-squares (SHELXL-97) \\
Function Minimized & $\sum w\left(\left|F_{o}\right|^{2}-\left|F_{c}\right|^{2}\right)^{2}$ \\
Least Squares Weight Scheme & $w=1 /\left[\sigma^{2}\left(F_{o}^{2}\right)+(0.0722 \mathrm{P})^{2}\right]$ where $\mathrm{P}=\left(F_{o}{ }^{2}+2 \mathrm{~F}_{\mathrm{c}}{ }^{2}\right) / 3$ \\
Anomalous Dispersion & All non-hydrogen atoms \\
No. Observations $\left(F_{o}>4 \sigma\left(F_{o}\right)\right)$ & 1687 \\
No. Variables & 228 \\
Reflection/Parameter Ratio & 12.04 \\
Residuals: $\mathrm{R}_{1} ; w \mathrm{w}_{2}$ & $0.0577 ; 0.11716$ \\
Goodness of Fit Indicator $(\mathrm{S})$ & 0.940 \\
\hline
\end{tabular}




\begin{tabular}{ll}
\hline Max Shift/Error in Final Cycle & 0.000 \\
Maximum peak in Final Diff. Map & $0.29 \mathrm{e} / \AA^{3}$ \\
Minimum peak in Final Diff. Map & $-0.23 \mathrm{e}^{-} / \AA^{3}$ \\
\hline
\end{tabular}

Table 5. Bond Lengths ( $\left({ }^{)}\right.$.

\begin{tabular}{llllll}
\hline Atom & Atom & Distance & Atom & Atom & Distance \\
\hline O1 & C1 & $1.217(3)$ & C1 & C5 & $1.502(4)$ \\
C1 & C2 & $1.508(4)$ & C2 & C3 & $1.348(4)$ \\
C2 & C7 & $1.467(4)$ & C3 & C13 & $1.469(4)$ \\
C3 & C4 & $1.516(4)$ & C4 & C5 & $1.352(4)$ \\
C4 & C6 & $1.492(4)$ & C5 & C19 & $1.467(4)$ \\
C7 & C8 & $1.396(4)$ & C7 & C12 & $1.398(4)$ \\
C8 & C9 & $1.386(4)$ & C9 & C10 & $1.372(4)$ \\
C10 & C11 & $1.382(4)$ & C11 & C12 & $1.375(4)$ \\
C13 & C18 & $1.398(4)$ & C13 & C14 & $1.398(4)$ \\
C14 & C15 & $1.376(4)$ & C15 & C16 & $1.385(4)$ \\
C16 & C17 & $1.378(4)$ & C17 & C18 & $1.376(4)$ \\
C19 & C24 & $1.397(4)$ & C19 & C20 & $1.401(4)$ \\
C20 & C21 & $1.384(4)$ & C21 & C22 & $1.378(4)$ \\
C22 & C23 & $1.381(4)$ & C23 & C24 & $1.382(4)$ \\
\hline
\end{tabular}

Table 6. Bond Angles ( ${ }^{\circ}$ ).

\begin{tabular}{llllllll}
\hline Atom & Atom & Atom & Angle & Atom & Atom & Atom & Angle \\
\hline O1 & C1 & C5 & $127.2(3)$ & O1 & C1 & C2 & $125.5(3)$ \\
C5 & C1 & C2 & $107.3(2)$ & C3 & C2 & C7 & $130.5(3)$ \\
C3 & C2 & C1 & $106.6(2)$ & C7 & C2 & C1 & $122.7(3)$ \\
C2 & C3 & C13 & $127.8(3)$ & C2 & C3 & C4 & $109.7(2)$ \\
C13 & C3 & C4 & $122.5(3)$ & C5 & C4 & C6 & $128.0(3)$ \\
C5 & C4 & C3 & $109.6(3)$ & C6 & C4 & C3 & $122.3(3)$ \\
C4 & C5 & C19 & $130.3(3)$ & C4 & C5 & C1 & $106.6(3)$ \\
C19 & C5 & C1 & $123.0(2)$ & C8 & C7 & C12 & $117.8(3)$ \\
C8 & C7 & C2 & $120.3(3)$ & C12 & C7 & C2 & $121.9(3)$ \\
C9 & C8 & C7 & $120.7(3)$ & C10 & C9 & C8 & $120.4(3)$ \\
C9 & C10 & C11 & $119.8(3)$ & C12 & C11 & C10 & $120.2(3)$ \\
C11 & C12 & C7 & $121.1(3)$ & C18 & C13 & C14 & $117.6(3)$ \\
C18 & C13 & C3 & $122.0(2)$ & C14 & C13 & C3 & $120.5(2)$ \\
C15 & C14 & C13 & $120.9(3)$ & C14 & C15 & C16 & $120.3(3)$ \\
C17 & C16 & C15 & $119.8(3)$ & C18 & C17 & C16 & $119.8(3)$ \\
C17 & C18 & C13 & $121.6(3)$ & C24 & C19 & C20 & $117.2(3)$ \\
C24 & C19 & C5 & $121.4(3)$ & C20 & C19 & C5 & $121.3(2)$ \\
C21 & C20 & C19 & $121.0(3)$ & C22 & C21 & C20 & $120.5(3)$ \\
C21 & C22 & C23 & $119.6(3)$ & C22 & C23 & C24 & $120.1(3)$ \\
C23 & C24 & C19 & $121.5(3)$ & & & & \\
\hline
\end{tabular}

Table 7. Torsion Angles ( ${ }^{\circ}$ ).

\begin{tabular}{llllllllll}
\hline Atom & Atom & Atom & Atom & Angle & Atom & Atom & Atom & Atom & Angle \\
\hline O1 & C1 & C2 & C3 & $175.1(2)$ & C5 & C1 & C2 & C3 & $-2.7(3)$ \\
O1 & C1 & C2 & C7 & $-1.9(4)$ & C5 & C1 & C2 & C7 & $-179.8(2)$ \\
C7 & C2 & C3 & C13 & $-4.6(5)$ & C1 & C2 & C3 & C13 & $178.7(2)$ \\
C7 & C2 & C3 & C4 & $178.0(2)$ & C1 & C2 & C3 & C4 & $1.3(3)$ \\
C2 & C3 & C4 & C5 & $0.8(3)$ & C13 & C3 & C4 & C5 & $-176.8(2)$ \\
C2 & C3 & C4 & C6 & $179.3(2)$ & C13 & C3 & C4 & C6 & $1.6(4)$ \\
C6 & C4 & C5 & C19 & $-3.7(5)$ & C3 & C4 & C5 & C19 & $174.6(2)$ \\
C6 & C4 & C5 & C1 & $179.2(2)$ & C3 & C4 & C5 & C1 & $-2.5(3)$ \\
O1 & C1 & C5 & C4 & $-174.6(2)$ & C2 & C1 & C5 & C4 & $3.3(3)$ \\
\hline
\end{tabular}




\begin{tabular}{llllllllll}
\hline O1 & C1 & C5 & C19 & $8.0(4)$ & C2 & C1 & C5 & C19 & $-174.2(2)$ \\
C3 & C2 & C7 & C8 & $145.0(3)$ & C1 & C2 & C7 & C8 & $-38.8(4)$ \\
C3 & C2 & C7 & C12 & $-37.0(4)$ & C1 & C2 & C7 & C12 & $139.3(3)$ \\
C12 & C7 & C8 & C9 & $1.9(4)$ & C2 & C7 & C8 & C9 & $180.0(3)$ \\
C7 & C8 & C9 & C10 & $-1.4(4)$ & C8 & C9 & C10 & C11 & $0.0(4)$ \\
C9 & C10 & C11 & C12 & $0.8(4)$ & C10 & C11 & C12 & C7 & $-0.3(4)$ \\
C8 & C7 & C12 & C11 & $-1.0(4)$ & C2 & C7 & C12 & C11 & $-179.1(3)$ \\
C2 & C3 & C13 & C18 & $120.8(3)$ & C4 & C3 & C13 & C18 & $-62.1(4)$ \\
C2 & C3 & C13 & C14 & $-59.1(4)$ & C4 & C3 & C13 & C14 & $118.1(3)$ \\
C18 & C13 & C14 & C15 & $1.2(4)$ & C3 & C13 & C14 & C15 & $-178.9(3)$ \\
C13 & C14 & C15 & C16 & $-0.5(5)$ & C14 & C15 & C16 & C17 & $-0.4(5)$ \\
C15 & C16 & C17 & C18 & $0.6(5)$ & C16 & C17 & C18 & C13 & $0.1(5)$ \\
C14 & C13 & C18 & C17 & $-1.0(4)$ & C3 & C13 & C18 & C17 & $179.1(3)$ \\
C4 & C5 & C19 & C24 & $147.4(3)$ & C1 & C5 & C19 & C24 & $-35.8(4)$ \\
C4 & C5 & C19 & C20 & $-35.6(4)$ & C1 & C5 & C19 & C20 & $141.1(3)$ \\
C24 & C19 & C20 & C21 & $0.1(4)$ & C5 & C19 & C20 & C21 & $-177.0(2)$ \\
C19 & C20 & C21 & C22 & $2.3(4)$ & C20 & C21 & C22 & C23 & $-2.3(4)$ \\
C21 & C22 & C23 & C24 & $0.0(4)$ & C22 & C23 & C24 & C19 & $2.5(4)$ \\
\hline
\end{tabular}

\section{X-Ray Crystallographic Data for Compound 11a.}

A brown rhombic crystal of $\mathrm{C}_{61} \mathrm{H}_{40} \mathrm{O}$ having approximate dimensions of $0.20 \times 0.11 \times 0.07 \mathrm{~mm}$ was mounted on a quartz fiber using Paratone $\mathrm{N}$ hydrocarbon oil. All measurements were made on a Siemens SMART-APEX ${ }^{1}$ CCD area detector with monochromatic radiation of wavelength $0.71073 \AA$.

Cell constants and an orientation matrix for data collection, obtained from a least-squares refinement using the measured positions of 2608 centered reflections with $I>10 \sigma(I)$ in the range $2.22^{\circ}<\theta<23.04^{\circ}$, corresponded to a primitive monoclinic cell with dimensions:

$$
\begin{array}{ll}
a=12.459(2) \AA & \alpha=90^{\circ} \quad V=4290.5(10) \AA^{3} \\
b=23.392(3) \AA & \beta=99.347(3)^{\circ}
\end{array}
$$

For $Z=4$ and $F . W .=788.93$, the calculated density is $1.221 \mathrm{~g} / \mathrm{cm}^{3}$. Based on a statistical analysis of intensity distribution, and the successful solution and refinement of the structure, the space group was determined to be $\mathrm{P} 21 / \mathrm{c}$. The data were collected at a temperature of $148 \mathrm{~K}$. Frames corresponding to an arbitrary hemisphere of data were collected using $\omega$ scans of $0.3^{\circ}$ counted for a total of 30 seconds per frame.

Data were integrated by the program SAINT with box parameters of $1.6 \times 1.6 \times 1.0^{\circ}$ to a maximum $\theta$ value of $23.29^{\circ}$. The data were corrected for Lorentz and polarization effects. The linear absorption coefficient, $\mu$, for $0.71073 \AA$ radiation is $0.071 \mathrm{~mm}^{-1}$. Data were analyzed for agreement and possible absorption using SADABS. A semi-empirical absorption correction based on 2.57 reflections with $I>$ $5 \sigma(I)$ was applied that resulted in normalized transmission factors ranging from 0.91 to 1 . Of the 18844 reflections that were collected, 6159 were unique $\left(R_{\text {int }}=0.0430\right)$; equivalent reflections were merged. No decay correction was deemed necessary.

The structure was solved by direct methods (SIR-97) and expanded using Fourier techniques. All nonhydrogen atoms were refined with anisotropic thermal parameters. Hydrogen atoms were located on the difference Fourier map, but were positioned using the HFIX command (idealized positions via a riding refinement). The final cycle of full-matrix least-squares refinement ${ }^{6}$ was based on 6159 reflections (all data) and 559 variable parameters and converged (largest parameter shift was 0.000 times its esd) with conventional unweighted and weighted agreement factors of $R_{1}=\Sigma\left\|F_{o}|-| F_{c}\right\| / \Sigma\left|F_{o}\right|=0.0432$ for 4303 data with $F_{o}>4 \sigma\left(F_{o}\right)$, and $w R_{2}=\left[\left(\Sigma w\left(\left|F_{o}\right|^{2}-\left|F_{c}\right|^{2}\right)^{2} / \Sigma w\left|F_{o}\right|^{2}\right)\right]^{1 / 2}=0.1108$ 
The standard deviation of an observation of unit weight $(S)^{7}$ was 1.017. Sheldrick weights ${ }^{6}$ were used; where applicable, weights were refined to convergence. The maximum and minimum peaks on the final difference Fourier map corresponded to 0.28 and $-0.21 \mathrm{e}^{-} / \AA^{3}$, respectively.

Table 8. Crystal Data.

\begin{tabular}{ll}
\hline Empirical Formula & $\mathrm{C}_{61} \mathrm{H}_{40} \mathrm{O}$ \\
Formula Weight & 788.93 \\
Crystal Color, Habit & brown, rhombic \\
Crystal Dimensions & $0.20 \mathrm{x} 0.11 \mathrm{x} 0.07 \mathrm{~mm}$ \\
Crystal System & monoclinic \\
Lattice Type & primitive \\
Lattice Parameters & $a=12.459(2) \AA ; b=23.392(3) \AA ; c=$ \\
& $14.920(2) \AA$ \\
& $\alpha=90^{\circ} ; \beta=99.347(3)^{\circ} ; \gamma=90^{\circ}$ \\
Space Group & $V=4290.5(10) \AA^{3}$ \\
$Z$ value & $\mathrm{P} 21 / \mathrm{c}$ \\
$d_{\text {calc }}$ & 4 \\
$F_{000}$ & $1.221 \mathrm{~g} / \mathrm{cm}^{3}$ \\
$\mu(0.71073 \AA$ radiation $)$ & 1656 \\
\hline
\end{tabular}

Table 9. Intensity Measurements.

\begin{tabular}{ll}
\hline Diffractometer & Siemens SMART-APEX CCD \\
Radiation & $\lambda=0.71073 \AA \AA$, graphite monochromated \\
Exposure Time & 30 seconds per frame. \\
Scan Type & $\omega(0.3$ degrees per frame $)$ \\
$\theta_{\max }$ & $23.29^{\circ}$ \\
Data Collection Temperature & $148 \mathrm{~K}$ \\
No. of Reflections Measured & Total: 18844, Unique: $6159\left(R_{\text {int }}=0.0430\right)$ \\
Corrections & Lorentz-polarization \\
& Absorption: $T_{\max }=1, T_{\min }=0.91$ \\
\hline
\end{tabular}

Table 10. Structure Solution and Refinement.

Structure Solution

Refinement

Function Minimized

Least Squares Weighting scheme

Anomalous Dispersion

No. Observations $\left(F_{o}>4 \sigma\left(F_{o}\right)\right)$

No. Variables

Reflection/Parameter Ratio

Residuals: $R_{1}$; w $R_{2}$

Goodness of Fit Indicator $(S)$

Max Shift/Error in Final Cycle

Maximum peak in Final Diff. Map

Minimum peak in Final Diff. Map
Direct (SIR-97)

Full-matrix least-squares (SHELXL-97)

$\sum w\left(\left|F_{o}\right|^{2}-\left|F_{c}\right|^{2}\right)^{2}$

$w=1 /\left[\sigma^{2}\left(F_{o}{ }^{2}\right)+(0.0510 \mathrm{P})^{2}+0.5992 \mathrm{P}\right]$ where $\mathrm{P}=\left(F_{o}{ }^{2}+2 \mathrm{~F}_{\mathrm{c}}{ }^{2}\right) / 3$

All non-hydrogen atoms

4303

559

11.02

$0.0432 ; 0.1108$

1.017

0.000

$0.28 \mathrm{e}^{-} / \AA^{3}$

$-0.21 \mathrm{e}^{-} / \AA^{3}$

Table 11. Bond Lengths ( $($ ).

\begin{tabular}{llllll}
\hline Atom & Atom & Distance & Atom & Atom & Distance \\
\hline O1 & C1 & $1.217(2)$ & C1 & C5 & $1.491(3)$ \\
C1 & C2 & $1.493(3)$ & C2 & C3 & $1.348(3)$ \\
C2 & C20 & $1.470(3)$ & C3 & C6 & $1.484(3)$ \\
C3 & C4 & $1.521(3)$ & C4 & C5 & $1.346(3)$ \\
C4 & C32 & $1.476(3)$ & C5 & C26 & $1.477(3)$ \\
C6 & C11 & $1.406(3)$ & C6 & C7 & $1.414(3)$
\end{tabular}




\begin{tabular}{|c|c|c|c|c|c|}
\hline C7 & C8 & $1.403(3)$ & C7 & C38 & $1.496(3)$ \\
\hline $\mathrm{C} 8$ & C9 & $1.405(3)$ & $\mathrm{C} 8$ & C44 & $1.498(3)$ \\
\hline $\mathrm{C} 9$ & $\mathrm{C} 10$ & $1.416(3)$ & $\mathrm{C} 9$ & $\mathrm{C} 12$ & $1.440(3)$ \\
\hline $\mathrm{C} 10$ & $\mathrm{C} 11$ & $1.394(3)$ & $\mathrm{C} 10$ & C50 & $1.492(3)$ \\
\hline C11 & C56 & $1.500(3)$ & C12 & $\mathrm{C} 13$ & $1.197(3)$ \\
\hline $\mathrm{C} 13$ & C14 & $1.441(3)$ & C14 & $\mathrm{C} 15$ & $1.382(3)$ \\
\hline C14 & C19 & $1.392(3)$ & C15 & $\mathrm{C} 16$ & $1.375(3)$ \\
\hline C16 & $\mathrm{C} 17$ & $1.372(4)$ & $\mathrm{C} 17$ & $\mathrm{C} 18$ & $1.369(4)$ \\
\hline C18 & C19 & $1.390(3)$ & $\mathrm{C} 20$ & $\mathrm{C} 25$ & $1.391(3)$ \\
\hline C20 & $\mathrm{C} 21$ & $1.398(3)$ & $\mathrm{C} 21$ & $\mathrm{C} 22$ & $1.377(3)$ \\
\hline $\mathrm{C} 22$ & $\mathrm{C} 23$ & $1.374(3)$ & $\mathrm{C} 23$ & $\mathrm{C} 24$ & $1.378(3)$ \\
\hline C24 & $\mathrm{C} 25$ & $1.372(3)$ & $\mathrm{C} 26$ & $\mathrm{C} 27$ & $1.375(3)$ \\
\hline $\mathrm{C} 26$ & $\mathrm{C} 31$ & $1.392(4)$ & $\mathrm{C} 27$ & $\mathrm{C} 28$ & $1.418(4)$ \\
\hline $\mathrm{C} 28$ & C29 & $1.365(5)$ & C29 & C30 & $1.346(5)$ \\
\hline C30 & C31 & $1.381(4)$ & C32 & C33 & $1.396(3)$ \\
\hline C32 & C37 & $1.399(3)$ & C33 & C34 & $1.377(3)$ \\
\hline C34 & C35 & $1.375(3)$ & C35 & C36 & $1.379(3)$ \\
\hline C36 & C37 & $1.377(3)$ & C38 & C43 & $1.386(3)$ \\
\hline C38 & C39 & $1.390(3)$ & C39 & C40 & $1.376(3)$ \\
\hline C40 & C41 & $1.382(3)$ & C41 & C42 & $1.367(3)$ \\
\hline C42 & C43 & $1.380(3)$ & C44 & C49 & $1.380(3)$ \\
\hline C44 & C45 & $1.392(3)$ & $\mathrm{C} 45$ & C46 & $1.394(3)$ \\
\hline $\mathrm{C} 46$ & C47 & $1.377(4)$ & C47 & $\mathrm{C} 48$ & $1.371(4)$ \\
\hline C48 & C49 & $1.385(3)$ & C50 & C51 & $1.380(3)$ \\
\hline $\mathrm{C} 50$ & C55 & $1.384(3)$ & C51 & C52 & $1.385(3)$ \\
\hline C52 & C53 & $1.365(4)$ & C53 & C54 & $1.364(4)$ \\
\hline C54 & C55 & $1.375(3)$ & C56 & C57 & $1.388(3)$ \\
\hline C56 & C61 & $1.388(3)$ & C57 & C58 & $1.382(3)$ \\
\hline C58 & C59 & $1.375(3)$ & C59 & C60 & $1.378(3)$ \\
\hline C60 & C61 & $1.385(3)$ & & & \\
\hline
\end{tabular}

Table 12. Bond Angles ( $\left.{ }^{\circ}\right)$.

\begin{tabular}{llllllll}
\hline Atom & Atom & Atom & Angle & Atom & Atom & Atom & Angle \\
\hline O1 & C1 & C5 & $125.2(2)$ & O1 & C1 & C2 & $127.3(2)$ \\
C5 & C1 & C2 & $107.50(17)$ & C3 & C2 & C20 & $130.6(2)$ \\
C3 & C2 & C1 & $106.52(18)$ & C20 & C2 & C1 & $122.84(18)$ \\
C2 & C3 & C6 & $126.33(19)$ & C2 & C3 & C4 & $109.86(18)$ \\
C6 & C3 & C4 & $123.45(17)$ & C5 & C4 & C32 & $126.55(19)$ \\
C5 & C4 & C3 & $108.49(18)$ & C32 & C4 & C3 & $124.90(18)$ \\
C4 & C5 & C26 & $132.1(2)$ & C4 & C5 & C1 & $107.48(18)$ \\
C26 & C5 & C1 & $119.81(18)$ & C11 & C6 & C7 & $120.88(18)$ \\
C11 & C6 & C3 & $120.40(18)$ & C7 & C6 & C3 & $118.67(17)$ \\
C8 & C7 & C6 & $119.66(18)$ & C8 & C7 & C38 & $120.81(18)$ \\
C6 & C7 & C38 & $119.53(18)$ & C7 & C8 & C9 & $119.39(18)$ \\
C7 & C8 & C44 & $121.21(18)$ & C9 & C8 & C44 & $119.33(18)$ \\
C8 & C9 & C10 & $120.61(19)$ & C8 & C9 & C12 & $121.78(19)$ \\
C10 & C9 & C12 & $117.52(18)$ & C11 & C10 & C9 & $120.07(18)$ \\
C11 & C10 & C50 & $121.12(18)$ & C9 & C10 & C50 & $118.75(18)$ \\
C10 & C11 & C6 & $119.33(19)$ & C10 & C11 & C56 & $120.45(18)$ \\
C6 & C11 & C56 & $120.21(18)$ & C13 & C12 & C9 & $171.6(2)$ \\
C12 & C13 & C14 & $173.0(2)$ & C15 & C14 & C19 & $119.2(2)$ \\
C15 & C14 & C13 & $121.0(2)$ & C19 & C14 & C13 & $119.7(2)$ \\
C16 & C15 & C14 & $120.7(2)$ & C17 & C16 & C15 & $120.2(3)$ \\
C18 & C17 & C16 & $119.9(2)$ & C17 & C18 & C19 & $120.7(3)$ \\
C18 & C19 & C14 & $119.4(2)$ & C25 & C20 & C21 & $117.9(2)$ \\
C25 & C20 & C2 & $122.32(19)$ & C21 & C20 & C2 & $119.76(19)$ \\
C22 & C21 & C20 & $120.9(2)$ & C23 & C22 & C21 & $120.1(2)$ \\
C22 & C23 & C24 & $119.8(2)$ & C25 & C24 & C23 & $120.4(2)$ \\
\hline
\end{tabular}




\begin{tabular}{llllllll}
\hline C24 & C25 & C20 & $120.8(2)$ & C27 & C26 & C31 & $118.2(2)$ \\
C27 & C26 & C5 & $123.9(2)$ & C31 & C26 & C5 & $117.8(2)$ \\
C26 & C27 & C28 & $119.3(3)$ & C29 & C28 & C27 & $119.6(3)$ \\
C30 & C29 & C28 & $122.0(3)$ & C29 & C30 & C31 & $118.5(3)$ \\
C30 & C31 & C26 & $122.3(3)$ & C33 & C32 & C37 & $117.4(2)$ \\
C33 & C32 & C4 & $122.80(19)$ & C37 & C32 & C4 & $119.81(19)$ \\
C34 & C33 & C32 & $121.1(2)$ & C35 & C34 & C33 & $120.3(2)$ \\
C34 & C35 & C36 & $119.9(2)$ & C37 & C36 & C35 & $119.9(2)$ \\
C36 & C37 & C32 & $121.4(2)$ & C43 & C38 & C39 & $118.30(19)$ \\
C43 & C38 & C7 & $120.36(19)$ & C39 & C38 & C7 & $121.32(19)$ \\
C40 & C39 & C38 & $120.6(2)$ & C39 & C40 & C41 & $120.3(2)$ \\
C42 & C41 & C40 & $119.5(2)$ & C41 & C42 & C43 & $120.5(2)$ \\
C42 & C43 & C38 & $120.7(2)$ & C49 & C44 & C45 & $118.6(2)$ \\
C49 & C44 & C8 & $121.4(2)$ & C45 & C44 & C8 & $119.9(2)$ \\
C44 & C45 & C46 & $120.7(2)$ & C47 & C46 & C45 & $119.4(3)$ \\
C48 & C47 & C46 & $120.3(2)$ & C47 & C48 & C49 & $120.3(3)$ \\
C44 & C49 & C48 & $120.7(2)$ & C51 & C50 & C55 & $118.8(2)$ \\
C51 & C50 & C10 & $119.6(2)$ & C55 & C50 & C10 & $121.6(2)$ \\
C50 & C51 & C52 & $120.2(2)$ & C53 & C52 & C51 & $120.2(3)$ \\
C54 & C53 & C52 & $119.9(2)$ & C53 & C54 & C55 & $120.6(3)$ \\
C54 & C55 & C50 & $120.3(2)$ & C57 & C56 & C61 & $118.02(19)$ \\
C57 & C56 & C11 & $120.18(18)$ & C61 & C56 & C11 & $121.79(18)$ \\
C58 & C57 & C56 & $121.3(2)$ & C59 & C58 & C57 & $120.0(2)$ \\
C58 & C59 & C60 & $119.7(2)$ & C59 & C60 & C61 & $120.3(2)$ \\
C60 & C61 & C56 & $120.7(2)$ & & & & \\
\hline
\end{tabular}

Table 13. Torsion Angles ( ${ }^{\circ}$ ).

\begin{tabular}{|c|c|c|c|c|c|c|c|c|c|}
\hline Atom & Atom & Atom & Atom & Angle & Atom & Atom & Atom & Atom & Angle \\
\hline $\mathrm{O} 1$ & $\mathrm{C} 1$ & $\mathrm{C} 2$ & C3 & $176.5(2)$ & C5 & $\mathrm{C} 1$ & $\mathrm{C} 2$ & C3 & $-1.9(2)$ \\
\hline $\mathrm{O} 1$ & $\mathrm{C} 1$ & $\mathrm{C} 2$ & $\mathrm{C} 20$ & $-5.8(3)$ & $\mathrm{C} 5$ & $\mathrm{C} 1$ & $\mathrm{C} 2$ & $\mathrm{C} 20$ & $175.75(19)$ \\
\hline $\mathrm{C} 20$ & $\mathrm{C} 2$ & $\mathrm{C} 3$ & C6 & $-4.4(4)$ & $\mathrm{C} 1$ & $\mathrm{C} 2$ & $\mathrm{C} 3$ & C6 & $173.04(18)$ \\
\hline C20 & $\mathrm{C} 2$ & $\mathrm{C} 3$ & $\mathrm{C} 4$ & $-177.7(2)$ & $\mathrm{C} 1$ & $\mathrm{C} 2$ & C3 & $\mathrm{C} 4$ & $-0.3(2)$ \\
\hline $\mathrm{C} 2$ & $\mathrm{C} 3$ & $\mathrm{C} 4$ & $\mathrm{C} 5$ & $2.6(2)$ & C6 & $\mathrm{C} 3$ & $\mathrm{C} 4$ & $\mathrm{C} 5$ & $-170.90(19)$ \\
\hline $\mathrm{C} 2$ & $\mathrm{C} 3$ & $\mathrm{C} 4$ & C32 & $179.96(19)$ & C6 & $\mathrm{C} 3$ & $\mathrm{C} 4$ & C32 & $6.4(3)$ \\
\hline C 32 & $\mathrm{C} 4$ & $\mathrm{C} 5$ & $\mathrm{C} 26$ & $-10.1(4)$ & $\mathrm{C} 3$ & $\mathrm{C} 4$ & $\mathrm{C} 5$ & $\mathrm{C} 26$ & $167.2(2)$ \\
\hline C32 & $\mathrm{C} 4$ & C5 & $\mathrm{C} 1$ & 179.01(19) & $\mathrm{C} 3$ & $\mathrm{C} 4$ & $\mathrm{C} 5$ & $\mathrm{C} 1$ & $-3.7(2)$ \\
\hline $\mathrm{O} 1$ & $\mathrm{C} 1$ & $\mathrm{C} 5$ & $\mathrm{C} 4$ & $-174.8(2)$ & $\mathrm{C} 2$ & $\mathrm{C} 1$ & $\mathrm{C} 5$ & $\mathrm{C} 4$ & $3.6(2)$ \\
\hline $\mathrm{O} 1$ & $\mathrm{C} 1$ & $\mathrm{C} 5$ & $\mathrm{C} 26$ & $12.9(3)$ & $\mathrm{C} 2$ & $\mathrm{C} 1$ & $\mathrm{C} 5$ & $\mathrm{C} 26$ & $-168.65(19)$ \\
\hline $\mathrm{C} 2$ & $\mathrm{C} 3$ & C6 & C11 & $119.0(2)$ & $\mathrm{C} 4$ & $\mathrm{C} 3$ & C6 & $\mathrm{C} 11$ & $-68.5(3)$ \\
\hline C2 & C3 & C6 & C7 & $-63.7(3)$ & $\mathrm{C} 4$ & $\mathrm{C} 3$ & C6 & C7 & $108.8(2)$ \\
\hline C11 & C6 & C7 & $\mathrm{C} 8$ & $-1.5(3)$ & $\mathrm{C} 3$ & C6 & $\mathrm{C} 7$ & $\mathrm{C} 8$ & $-178.83(18)$ \\
\hline C11 & C6 & C7 & C38 & $178.43(18)$ & C3 & C6 & C7 & C38 & $1.1(3)$ \\
\hline C6 & $\mathrm{C} 7$ & $\mathrm{C} 8$ & C9 & $-0.8(3)$ & C38 & $\mathrm{C} 7$ & $\mathrm{C} 8$ & C9 & $179.24(19)$ \\
\hline C6 & $\mathrm{C} 7$ & $\mathrm{C} 8$ & C44 & $175.90(19)$ & C38 & $\mathrm{C} 7$ & $\mathrm{C} 8$ & C44 & $-4.1(3)$ \\
\hline C7 & C8 & C9 & $\mathrm{C} 10$ & $2.1(3)$ & C44 & C8 & C9 & $\mathrm{C} 10$ & $-174.61(19)$ \\
\hline C7 & $\mathrm{C} 8$ & C9 & $\mathrm{C} 12$ & $178.48(19)$ & C44 & $\mathrm{C} 8$ & C9 & $\mathrm{C} 12$ & $1.7(3)$ \\
\hline C8 & C9 & $\mathrm{C} 10$ & $\mathrm{C} 11$ & $-1.2(3)$ & $\mathrm{C} 12$ & C9 & $\mathrm{C} 10$ & $\mathrm{C} 11$ & $-177.66(19)$ \\
\hline $\mathrm{C} 8$ & $\mathrm{C} 9$ & $\mathrm{C} 10$ & C50 & $176.21(19)$ & $\mathrm{C} 12$ & C9 & $\mathrm{C} 10$ & C50 & $-0.3(3)$ \\
\hline C9 & $\mathrm{C} 10$ & C11 & C6 & $-1.1(3)$ & C50 & $\mathrm{C} 10$ & C11 & C6 & $-178.47(19)$ \\
\hline C9 & $\mathrm{C} 10$ & C11 & C56 & 177.23(19) & C50 & $\mathrm{C} 10$ & C11 & C56 & $-0.1(3)$ \\
\hline C7 & C6 & C11 & $\mathrm{C} 10$ & $2.5(3)$ & C3 & C6 & C11 & $\mathrm{C} 10$ & $179.75(19)$ \\
\hline C7 & C6 & C11 & C56 & $-175.87(19)$ & $\mathrm{C} 3$ & C6 & C11 & C56 & $1.4(3)$ \\
\hline C8 & C9 & C12 & $\mathrm{C} 13$ & $-145.4(15)$ & $\mathrm{C} 10$ & C9 & C12 & $\mathrm{C} 13$ & $31.0(16)$ \\
\hline C9 & $\mathrm{C} 12$ & $\mathrm{C} 13$ & C14 & $35(3)$ & $\mathrm{C} 12$ & $\mathrm{C} 13$ & C14 & C15 & 107.1(19) \\
\hline $\mathrm{C} 12$ & $\mathrm{C} 13$ & $\mathrm{C} 14$ & C19 & $-68(2)$ & C19 & C14 & C15 & $\mathrm{C} 16$ & $1.2(4)$ \\
\hline $\mathrm{C} 13$ & C14 & C15 & $\mathrm{C} 16$ & $-174.3(2)$ & C14 & C15 & C16 & C17 & $-0.6(5)$ \\
\hline $\mathrm{C} 15$ & C16 & C17 & $\mathrm{C} 18$ & $-0.5(5)$ & C16 & C17 & $\mathrm{C} 18$ & C19 & $1.1(5)$ \\
\hline $\mathrm{C} 17$ & $\mathrm{C} 18$ & C19 & C14 & $-0.5(4)$ & C15 & C14 & C19 & $\mathrm{C} 18$ & $-0.6(4)$ \\
\hline
\end{tabular}




\begin{tabular}{|c|c|c|c|c|c|c|c|c|c|}
\hline $\mathrm{C} 13$ & C14 & C19 & $\mathrm{C} 18$ & $175.0(2)$ & $\mathrm{C} 3$ & $\mathrm{C} 2$ & $\mathrm{C} 20$ & $\mathrm{C} 25$ & $-33.7(4)$ \\
\hline $\mathrm{C} 1$ & $\mathrm{C} 2$ & $\mathrm{C} 20$ & $\mathrm{C} 25$ & $149.3(2)$ & $\mathrm{C} 3$ & $\mathrm{C} 2$ & $\mathrm{C} 20$ & $\mathrm{C} 21$ & $145.3(2)$ \\
\hline $\mathrm{C} 1$ & $\mathrm{C} 2$ & $\mathrm{C} 20$ & $\mathrm{C} 21$ & $-31.8(3)$ & $\mathrm{C} 25$ & $\mathrm{C} 20$ & $\mathrm{C} 21$ & $\mathrm{C} 22$ & $0.6(3)$ \\
\hline $\mathrm{C} 2$ & $\mathrm{C} 20$ & $\mathrm{C} 21$ & $\mathrm{C} 22$ & $-178.5(2)$ & $\mathrm{C} 20$ & $\mathrm{C} 21$ & $\mathrm{C} 22$ & $\mathrm{C} 23$ & $0.2(4)$ \\
\hline $\mathrm{C} 21$ & $\mathrm{C} 22$ & $\mathrm{C} 23$ & $\mathrm{C} 24$ & $-1.4(4)$ & $\mathrm{C} 22$ & $\mathrm{C} 23$ & $\mathrm{C} 24$ & $\mathrm{C} 25$ & $1.8(4)$ \\
\hline $\mathrm{C} 23$ & $\mathrm{C} 24$ & $\mathrm{C} 25$ & $\mathrm{C} 20$ & $-1.0(3)$ & $\mathrm{C} 21$ & $\mathrm{C} 20$ & $\mathrm{C} 25$ & $\mathrm{C} 24$ & $-0.1(3)$ \\
\hline $\mathrm{C} 2$ & $\mathrm{C} 20$ & $\mathrm{C} 25$ & $\mathrm{C} 24$ & $178.9(2)$ & $\mathrm{C} 4$ & C5 & $\mathrm{C} 26$ & $\mathrm{C} 27$ & $90.5(3)$ \\
\hline $\mathrm{C} 1$ & $\mathrm{C} 5$ & $\mathrm{C} 26$ & $\mathrm{C} 27$ & $-99.5(3)$ & $\mathrm{C} 4$ & C5 & $\mathrm{C} 26$ & $\mathrm{C} 31$ & $-94.2(3)$ \\
\hline $\mathrm{C} 1$ & $\mathrm{C} 5$ & $\mathrm{C} 26$ & $\mathrm{C} 31$ & $75.9(3)$ & C31 & $\mathrm{C} 26$ & $\mathrm{C} 27$ & $\mathrm{C} 28$ & $0.6(4)$ \\
\hline C5 & $\mathrm{C} 26$ & $\mathrm{C} 27$ & $\mathrm{C} 28$ & $175.9(2)$ & $\mathrm{C} 26$ & $\mathrm{C} 27$ & $\mathrm{C} 28$ & $\mathrm{C} 29$ & $-0.6(5)$ \\
\hline $\mathrm{C} 27$ & $\mathrm{C} 28$ & C29 & $\mathrm{C} 30$ & $0.4(5)$ & $\mathrm{C} 28$ & C29 & $\mathrm{C} 30$ & $\mathrm{C} 31$ & $-0.2(5)$ \\
\hline C29 & C30 & C31 & $\mathrm{C} 26$ & $0.2(5)$ & $\mathrm{C} 27$ & $\mathrm{C} 26$ & C31 & $\mathrm{C} 30$ & $-0.4(4)$ \\
\hline C5 & $\mathrm{C} 26$ & C31 & $\mathrm{C} 30$ & $-176.0(2)$ & $\mathrm{C} 5$ & $\mathrm{C} 4$ & $\mathrm{C} 32$ & $\mathrm{C} 33$ & $140.1(2)$ \\
\hline C3 & $\mathrm{C} 4$ & C32 & C33 & $-36.7(3)$ & $\mathrm{C} 5$ & $\mathrm{C} 4$ & C32 & C37 & $-38.2(3)$ \\
\hline $\mathrm{C} 3$ & $\mathrm{C} 4$ & C32 & C37 & $144.9(2)$ & C37 & C32 & C33 & C34 & $0.0(3)$ \\
\hline $\mathrm{C} 4$ & C32 & C33 & C34 & $-178.37(19)$ & C32 & C33 & C34 & C35 & $0.6(3)$ \\
\hline C33 & C34 & C35 & C36 & $-1.1(4)$ & C34 & C35 & C36 & C37 & $0.9(4)$ \\
\hline C35 & C36 & C37 & C32 & $-0.3(4)$ & C33 & C32 & C37 & C36 & $-0.2(3)$ \\
\hline $\mathrm{C} 4$ & C32 & C37 & C36 & $178.3(2)$ & C8 & $\mathrm{C} 7$ & C 38 & $\mathrm{C} 43$ & $-60.2(3)$ \\
\hline C6 & C7 & C38 & C43 & $119.8(2)$ & $\mathrm{C} 8$ & $\mathrm{C} 7$ & C38 & C39 & $121.6(2)$ \\
\hline C6 & C7 & C38 & C39 & $-58.4(3)$ & C43 & C38 & C39 & C40 & $-2.9(3)$ \\
\hline C7 & C38 & C39 & $\mathrm{C} 40$ & $175.3(2)$ & C38 & C39 & $\mathrm{C} 40$ & C41 & $0.8(4)$ \\
\hline C39 & C40 & C41 & $\mathrm{C} 42$ & $1.9(4)$ & $\mathrm{C} 40$ & C41 & $\mathrm{C} 42$ & $\mathrm{C} 43$ & $-2.4(4)$ \\
\hline C41 & C42 & $\mathrm{C} 43$ & C38 & $0.2(4)$ & C39 & C38 & C43 & C42 & $2.4(3)$ \\
\hline C7 & C38 & C43 & C42 & $-175.9(2)$ & C7 & C8 & C44 & C49 & $121.0(2)$ \\
\hline C9 & $\mathrm{C} 8$ & C44 & C49 & $-62.3(3)$ & $\mathrm{C} 7$ & $\mathrm{C} 8$ & C44 & C45 & $-60.1(3)$ \\
\hline C9 & $\mathrm{C} 8$ & C44 & C45 & $116.6(2)$ & C49 & C44 & C45 & C46 & $0.3(3)$ \\
\hline C8 & C44 & $\mathrm{C} 45$ & $\mathrm{C} 46$ & $-178.7(2)$ & C44 & $\mathrm{C} 45$ & $\mathrm{C} 46$ & $\mathrm{C} 47$ & $-0.8(3)$ \\
\hline C45 & C46 & C47 & C48 & $0.7(4)$ & C46 & C47 & C48 & C49 & $-0.1(4)$ \\
\hline $\mathrm{C} 45$ & C44 & C49 & $\mathrm{C} 48$ & $0.4(3)$ & $\mathrm{C} 8$ & $\mathrm{C} 44$ & C49 & C48 & $179.3(2)$ \\
\hline C47 & $\mathrm{C} 48$ & C49 & C44 & $-0.5(4)$ & C11 & $\mathrm{C} 10$ & C50 & C51 & $107.1(2)$ \\
\hline C9 & $\mathrm{C} 10$ & C50 & C51 & $-70.3(3)$ & C11 & $\mathrm{C} 10$ & C50 & C55 & $-72.3(3)$ \\
\hline C9 & $\mathrm{C} 10$ & C50 & C55 & $110.4(2)$ & C55 & C50 & C51 & C52 & $0.7(3)$ \\
\hline $\mathrm{C} 10$ & C50 & C51 & C52 & $-178.7(2)$ & C50 & C51 & C52 & C53 & $0.1(4)$ \\
\hline C51 & C52 & C53 & C54 & $-1.1(4)$ & C52 & C53 & C54 & C55 & $1.3(4)$ \\
\hline C53 & C54 & C55 & C50 & $-0.5(4)$ & C51 & C50 & C55 & C54 & $-0.5(3)$ \\
\hline $\mathrm{C} 10$ & C50 & C55 & C54 & $178.8(2)$ & $\mathrm{C} 10$ & C11 & C56 & C57 & $-59.2(3)$ \\
\hline C6 & C11 & C56 & C57 & 119.1(2) & $\mathrm{C} 10$ & C11 & C56 & C61 & $122.0(2)$ \\
\hline C6 & C11 & C56 & C61 & $-59.6(3)$ & C61 & C56 & C57 & C58 & $-0.6(3)$ \\
\hline $\mathrm{C} 11$ & C56 & C57 & C58 & $-179.4(2)$ & C56 & C57 & C58 & C59 & $0.4(3)$ \\
\hline C57 & C58 & C59 & C60 & $-0.2(4)$ & C58 & C59 & C60 & C61 & $0.2(4)$ \\
\hline C59 & C60 & C61 & C56 & $-0.4(3)$ & C57 & C56 & C61 & C60 & $0.6(3)$ \\
\hline $\mathrm{C} 11$ & C56 & C61 & C60 & $179.4(2)$ & & & & & \\
\hline
\end{tabular}

\section{X-Ray Crystallographic Data for Compound 16.}

A purple rhombic crystal of $\mathrm{C}_{27} \mathrm{H}_{22} \mathrm{O}_{3}$ having approximate dimensions of $0.20 \times 0.12 \times 0.10 \mathrm{~mm}$ was mounted on a quartz fiber using Paratone $\mathrm{N}$ hydrocarbon oil. All measurements were made on a BrukerSiemens SMART ${ }^{1}$ CCD area detector with monochromatic radiation of wavelength $0.71073 \AA$.

Cell constants and an orientation matrix for data collection, obtained from a least-squares refinement using the measured positions of 1563 centered reflections with $I>10 \sigma(I)$ in the range $3.33^{\circ}<\theta<22.4^{\circ}$, corresponded to a primitive monoclinic cell with dimensions:

$$
\begin{array}{ll}
a=8.976(4) \AA & \alpha=90^{\circ} \quad V=2033.9(17) \AA^{3} \\
b=24.230(12) \AA & \beta=111.058(6)^{\circ} \\
c=10.021(5) \AA & \gamma=90^{\circ}
\end{array}
$$

For $Z=4$ and $F . W .=394.45$, the calculated density is $1.288 \mathrm{~g} / \mathrm{cm}^{3}$. Based on a statistical analysis of 
intensity distribution, and the successful solution and refinement of the structure, the space group was determined to be $\mathrm{P} 2_{1} / \mathrm{a}$ (\#14). The data were collected at a temperature of $143 \mathrm{~K}$. Frames corresponding to an arbitrary hemisphere of data were collected using $\omega$ scans of $0.3^{\circ}$ counted for a total of 40 seconds per frame.

Data were integrated by the program SAINT with box parameters of $1.6 \times 1.6 \times 1.0^{\circ}$ to a maximum $\theta$ value of $23.44^{\circ}$. The data were corrected for Lorentz and polarization effects. The linear absorption coefficient, $\mu$, for $0.71073 \AA$ radiation is $0.083 \mathrm{~mm}^{-1}$. Data were analyzed for agreement and possible absorption using SADABS. A semi-empirical absorption correction based on 6.09 reflections with $I>$ $5 \sigma(I)$ was applied that resulted in normalized transmission factors ranging from 0.87 to 0.99 . Of the 9074 reflections that were collected, 2907 were unique $\left(R_{\text {int }}=0.0655\right)$; equivalent reflections were merged. No decay correction was deemed necessary.

The structure was solved by direct methods (SIR-2002) and expanded using Fourier techniques. All nonhydrogen atoms were refined with anisotropic thermal parameters. Hydrogen atoms were located using the HFIX command (idealized positions via a riding refinement). A single torsional parameter about the $\mathrm{X}-\mathrm{C}$ or X-O bond was refined in the case of methyl or hydroxyl groups. The final cycle of full-matrix least-squares refinement ${ }^{6}$ was based on 2907 reflections (all data) and 274 variable parameters and converged (largest parameter shift was 0.000 times its esd) with conventional unweighted and weighted agreement factors of $R_{1}=\Sigma|| F_{o}|-| F_{c} \| / \Sigma\left|F_{o}\right|=0.0511$ for 1967 data with $F_{o}>4 \sigma\left(F_{o}\right)$, and $\mathrm{w} R_{2}=[(\Sigma w$ $\left.\left.\left(\left|F_{o}\right|^{2}-\left|F_{c}\right|^{2}\right)^{2} / \Sigma w\left|F_{o}\right|^{2}\right)\right]^{1 / 2}=0.1419$.

The standard deviation of an observation of unit weight $(S)^{7}$ was 0.975 . Sheldrick weights ${ }^{6}$ were used; where applicable, weights were refined to convergence. The maximum and minimum peaks on the final difference Fourier map corresponded to 0.19 and $-0.20 \mathrm{e}^{-} / \AA^{3}$, respectively.

\section{Table 14. Crystal Data.}

\begin{tabular}{ll}
\hline Empirical Formula & $\mathrm{C}_{27} \mathrm{H}_{22} \mathrm{O}_{3}$ \\
Formula Weight & 394.45 \\
Crystal Color, Habit & purple, rhombic \\
Crystal Dimensions & $0.20 \times 0.12 \times 0.10 \mathrm{~mm}$ \\
Crystal System & Monoclinic \\
Lattice Type & Primitive \\
Lattice Parameters & $a=8.976(4) \AA ; b=24.230(12) \AA ; c=10.021(5) \AA$ \\
& $\alpha=90^{\circ} ; \beta=111.058(6)^{\circ} ; \gamma=90^{\circ}$ \\
& $V=2033.9(17) \AA^{3}$ \\
Space Group & $\mathrm{P} 21 / \mathrm{a}$ \\
$Z$ value & 4 \\
$d_{\text {calc }}$ & $1.288 \mathrm{~g} / \mathrm{cm}^{3}$ \\
$F_{000}$ & 832 \\
$\mu(0.71073 \AA$ radiation $)$ & $0.08 \mathrm{~cm}^{-1}$ \\
\hline
\end{tabular}

Table 15. Intensity Measurements.

\begin{tabular}{ll}
\hline Diffractometer & Bruker-Siemens SMART CCD \\
Radiation & $\lambda=0.71073 \AA$, graphite monochromated \\
Exposure Time & 40 seconds per frame. \\
Scan Type & $\omega(0.3$ degrees per frame $)$ \\
$\theta_{\max }$ & $23.44^{\circ}$ \\
Data Collection Temperature & $143 \mathrm{~K}$ \\
No. of Reflections Measured & Total: 9074 ; Unique: $2907\left(R_{\mathrm{int}}=0.0655\right)$ \\
Corrections & Lorentz-polarization \\
& Absorption: $T_{\max }=0.99, T_{\min }=0.87$ \\
\hline
\end{tabular}

Table 16. Structure Solution and Refinement.

\begin{tabular}{ll}
\hline Structure Solution & Direct (SHELXS-97) \\
Refinement & Full-matrix least-squares \\
Function Minimized & $\sum w\left(\left|F_{o}\right|^{2}-\mid F_{c}{ }^{2}\right)^{2}$ \\
\hline
\end{tabular}




\begin{tabular}{ll}
\hline Least Squares Weighting scheme & $w=1 /\left[\sigma^{2}\left(F_{o}{ }^{2}\right)+(0.0000 \mathrm{P})^{2}\right]$ where $\mathrm{P}=\left(F_{o}{ }^{2}+2 \mathrm{~F}_{\mathrm{c}}{ }^{2}\right) / 3$ \\
Anomalous Dispersion & All non-hydrogen atoms \\
No. Observations $\left(F_{o}>4 \sigma\left(F_{o}\right)\right)$ & 1967 \\
No. Variables & 274 \\
Reflection/Parameter Ratio & 10.61 \\
Residuals: $R_{1} ; \mathrm{w} R_{2}$ & $0.0511 ; 0.1419$ \\
Goodness of Fit Indicator $(S)$ & 0.975 \\
Max Shift/Error in Final Cycle & 0.000 \\
Maximum peak in Final Diff. Map & $0.19 \mathrm{e}^{-} / \AA^{3}$ \\
Minimum peak in Final Diff. Map & $-0.20 \mathrm{e}^{-} / \AA^{3}$ \\
\hline
\end{tabular}

Table 17. Bond Lengths ( $\AA$ ).

\begin{tabular}{llllll}
\hline Atom & Atom & Distance & Atom & Atom & Distance \\
\hline O1 & C2 & $1.208(3)$ & O2 & C10 & $1.216(3)$ \\
O3 & C14 & $1.420(3)$ & O3 & C15 & $1.428(3)$ \\
C1 & C2 & $1.505(4)$ & C2 & C3 & $1.503(4)$ \\
C3 & C8 & $1.389(4)$ & C3 & C4 & $1.391(4)$ \\
C4 & C5 & $1.379(4)$ & C5 & C6 & $1.391(4)$ \\
C6 & C7 & $1.397(3)$ & C6 & C9 & $1.469(4)$ \\
C7 & C8 & $1.381(4)$ & C9 & C13 & $1.344(3)$ \\
C9 & C10 & $1.514(4)$ & C10 & C11 & $1.511(4)$ \\
C11 & C12 & $1.354(3)$ & C11 & C16 & $1.473(4)$ \\
C12 & C22 & $1.477(4)$ & C12 & C13 & $1.511(4)$ \\
C13 & C14 & $1.497(4)$ & C16 & C21 & $1.388(3)$ \\
C16 & C17 & $1.397(3)$ & C17 & C18 & $1.379(4)$ \\
C18 & C19 & $1.380(4)$ & C19 & C20 & $1.379(4)$ \\
C20 & C21 & $1.388(4)$ & C22 & C23 & $1.386(4)$ \\
C22 & C27 & $1.393(4)$ & C23 & C24 & $1.384(4)$ \\
C24 & C25 & $1.383(4)$ & C25 & C26 & $1.376(4)$ \\
C26 & C27 & $1.379(4)$ & & & \\
\hline
\end{tabular}

Table 18. Bond Angles $\left({ }^{\circ}\right)$.

\begin{tabular}{llllllll}
\hline Atom & Atom & Atom & Angle & Atom & Atom & Atom & Angle \\
\hline C14 & O3 & C15 & $111.3(2)$ & O1 & C2 & C3 & $120.5(3)$ \\
O1 & C2 & C1 & $120.8(3)$ & C3 & C2 & C1 & $118.8(3)$ \\
C8 & C3 & C4 & $118.5(2)$ & C8 & C3 & C2 & $122.2(3)$ \\
C4 & C3 & C2 & $119.3(3)$ & C5 & C4 & C3 & $120.9(3)$ \\
C4 & C5 & C6 & $121.0(2)$ & C5 & C6 & C7 & $117.9(2)$ \\
C5 & C6 & C9 & $122.4(2)$ & C7 & C6 & C9 & $119.7(2)$ \\
C8 & C7 & C6 & $121.1(2)$ & C7 & C8 & C3 & $120.6(3)$ \\
C13 & C9 & C6 & $129.5(2)$ & C13 & C9 & C10 & $106.3(2)$ \\
C6 & C9 & C10 & $124.1(2)$ & O2 & C10 & C11 & $127.5(2)$ \\
O2 & C10 & C9 & $125.5(2)$ & C11 & C10 & C9 & $107.0(2)$ \\
C12 & C11 & C16 & $129.1(2)$ & C12 & C11 & C10 & $106.8(2)$ \\
C16 & C11 & C10 & $124.0(2)$ & C11 & C12 & C22 & $129.0(2)$ \\
C11 & C12 & C13 & $109.2(2)$ & C22 & C12 & C13 & $121.6(2)$ \\
C9 & C13 & C14 & $127.4(2)$ & C9 & C13 & C12 & $110.6(2)$ \\
C14 & C13 & C12 & $121.9(2)$ & O3 & C14 & C13 & $108.8(2)$ \\
C21 & C16 & C17 & $117.9(2)$ & C21 & C16 & C11 & $120.8(2)$ \\
C17 & C16 & C11 & $121.3(2)$ & C18 & C17 & C16 & $120.8(3)$ \\
C17 & C18 & C19 & $120.4(3)$ & C20 & C19 & C18 & $119.7(3)$ \\
C19 & C20 & C21 & $119.8(3)$ & C20 & C21 & C16 & $121.2(2)$ \\
C23 & C22 & C27 & $118.3(2)$ & C23 & C22 & C12 & $120.4(2)$ \\
C27 & C22 & C12 & $121.3(2)$ & C24 & C23 & C22 & $120.9(2)$ \\
C25 & C24 & C23 & $120.1(3)$ & C26 & C25 & C24 & $119.5(3)$ \\
C25 & C26 & C27 & $120.6(3)$ & C26 & C27 & C22 & $120.7(3)$ \\
\hline
\end{tabular}


Table 19. Torsion Angles ( ${ }^{\circ}$ ).

\begin{tabular}{|c|c|c|c|c|c|c|c|c|c|}
\hline Atom & Atom & Atom & Atom & Angle & Atom & Atom & Atom & Atom & Angle \\
\hline O1 & $\mathrm{C} 2$ & C3 & $\mathrm{C} 8$ & $-173.3(3)$ & $\mathrm{C} 1$ & $\mathrm{C} 2$ & C3 & $\mathrm{C} 8$ & $6.0(4)$ \\
\hline $\mathrm{O} 1$ & $\mathrm{C} 2$ & $\mathrm{C} 3$ & $\mathrm{C} 4$ & $5.0(4)$ & $\mathrm{C} 1$ & $\mathrm{C} 2$ & $\mathrm{C} 3$ & $\mathrm{C} 4$ & $-175.7(3)$ \\
\hline $\mathrm{C} 8$ & $\mathrm{C} 3$ & $\mathrm{C} 4$ & C5 & $-0.2(4)$ & $\mathrm{C} 2$ & C3 & $\mathrm{C} 4$ & C5 & $-178.5(2)$ \\
\hline C3 & $\mathrm{C} 4$ & C5 & C6 & $0.9(4)$ & $\mathrm{C} 4$ & C5 & C6 & C7 & $-1.0(4)$ \\
\hline $\mathrm{C} 4$ & $\mathrm{C} 5$ & C6 & C9 & $-179.5(2)$ & $\mathrm{C} 5$ & C6 & C7 & $\mathrm{C} 8$ & $0.2(4)$ \\
\hline C9 & C6 & C7 & C8 & $178.8(2)$ & C6 & C7 & C8 & $\mathrm{C} 3$ & $0.5(4)$ \\
\hline $\mathrm{C} 4$ & $\mathrm{C} 3$ & $\mathrm{C} 8$ & $\mathrm{C} 7$ & $-0.5(4)$ & $\mathrm{C} 2$ & $\mathrm{C} 3$ & $\mathrm{C} 8$ & $\mathrm{C} 7$ & $177.8(2)$ \\
\hline C5 & C6 & C9 & $\mathrm{C} 13$ & $-145.0(3)$ & $\mathrm{C} 7$ & C6 & C9 & $\mathrm{C} 13$ & $36.5(4)$ \\
\hline C5 & C6 & C9 & $\mathrm{C} 10$ & $38.1(4)$ & C7 & C6 & C9 & $\mathrm{C} 10$ & $-140.4(3)$ \\
\hline $\mathrm{C} 13$ & C9 & $\mathrm{C} 10$ & $\mathrm{O} 2$ & $-177.1(3)$ & C6 & C9 & $\mathrm{C} 10$ & $\mathrm{O} 2$ & $0.4(4)$ \\
\hline $\mathrm{C} 13$ & C9 & $\mathrm{C} 10$ & C11 & $1.0(3)$ & C6 & C9 & $\mathrm{C} 10$ & $\mathrm{C} 11$ & $178.5(2)$ \\
\hline $\mathrm{O} 2$ & $\mathrm{C} 10$ & C11 & $\mathrm{C} 12$ & $177.0(3)$ & C9 & $\mathrm{C} 10$ & $\mathrm{C} 11$ & $\mathrm{C} 12$ & $-1.1(3)$ \\
\hline $\mathrm{O} 2$ & $\mathrm{C} 10$ & C11 & C16 & $1.1(4)$ & C9 & $\mathrm{C} 10$ & $\mathrm{C} 11$ & C16 & $-176.9(2)$ \\
\hline C16 & C11 & $\mathrm{C} 12$ & C22 & $-7.8(5)$ & $\mathrm{C} 10$ & C11 & $\mathrm{C} 12$ & $\mathrm{C} 22$ & 176.6(3) \\
\hline C16 & $\mathrm{C} 11$ & $\mathrm{C} 12$ & $\mathrm{C} 13$ & $176.3(2)$ & $\mathrm{C} 10$ & $\mathrm{C} 11$ & $\mathrm{C} 12$ & $\mathrm{C} 13$ & $0.7(3)$ \\
\hline C6 & C9 & $\mathrm{C} 13$ & C14 & $5.3(5)$ & $\mathrm{C} 10$ & C9 & $\mathrm{C} 13$ & $\mathrm{C} 14$ & $-177.4(2)$ \\
\hline C6 & C9 & $\mathrm{C} 13$ & $\mathrm{C} 12$ & $-177.9(2)$ & $\mathrm{C} 10$ & C9 & $\mathrm{C} 13$ & $\mathrm{C} 12$ & $-0.6(3)$ \\
\hline C11 & $\mathrm{C} 12$ & C13 & C9 & $-0.1(3)$ & $\mathrm{C} 22$ & $\mathrm{C} 12$ & $\mathrm{C} 13$ & C9 & $-176.3(2)$ \\
\hline C11 & $\mathrm{C} 12$ & $\mathrm{C} 13$ & C14 & $176.9(2)$ & $\mathrm{C} 22$ & $\mathrm{C} 12$ & $\mathrm{C} 13$ & C14 & $0.7(4)$ \\
\hline C15 & $\mathrm{O} 3$ & C14 & $\mathrm{C} 13$ & $-158.9(2)$ & C9 & $\mathrm{C} 13$ & C14 & $\mathrm{O} 3$ & $-92.0(3)$ \\
\hline C12 & $\mathrm{C} 13$ & C14 & $\mathrm{O} 3$ & $91.6(3)$ & $\mathrm{C} 12$ & $\mathrm{C} 11$ & $\mathrm{C} 16$ & $\mathrm{C} 21$ & $-26.7(4)$ \\
\hline C10 & C11 & C16 & $\mathrm{C} 21$ & $148.3(3)$ & $\mathrm{C} 12$ & C11 & C16 & C17 & $152.8(3)$ \\
\hline C10 & $\mathrm{C} 11$ & C16 & $\mathrm{C} 17$ & $-32.2(4)$ & $\mathrm{C} 21$ & $\mathrm{C} 16$ & $\mathrm{C} 17$ & $\mathrm{C} 18$ & $1.3(4)$ \\
\hline C11 & $\mathrm{C} 16$ & $\mathrm{C} 17$ & $\mathrm{C} 18$ & $-178.2(2)$ & $\mathrm{C} 16$ & $\mathrm{C} 17$ & $\mathrm{C} 18$ & C19 & $0.3(4)$ \\
\hline $\mathrm{C} 17$ & $\mathrm{C} 18$ & C19 & $\mathrm{C} 20$ & $-1.2(4)$ & $\mathrm{C} 18$ & C19 & $\mathrm{C} 20$ & $\mathrm{C} 21$ & $0.4(4)$ \\
\hline C19 & $\mathrm{C} 20$ & $\mathrm{C} 21$ & C16 & $1.2(4)$ & $\mathrm{C} 17$ & $\mathrm{C} 16$ & $\mathrm{C} 21$ & $\mathrm{C} 20$ & $-2.0(4)$ \\
\hline C11 & $\mathrm{C} 16$ & $\mathrm{C} 21$ & $\mathrm{C} 20$ & $177.5(2)$ & $\mathrm{C} 11$ & $\mathrm{C} 12$ & $\mathrm{C} 22$ & $\mathrm{C} 23$ & $-54.7(4)$ \\
\hline $\mathrm{C} 13$ & $\mathrm{C} 12$ & $\mathrm{C} 22$ & $\mathrm{C} 23$ & $120.7(3)$ & $\mathrm{C} 11$ & $\mathrm{C} 12$ & $\mathrm{C} 22$ & $\mathrm{C} 27$ & $126.4(3)$ \\
\hline C13 & $\mathrm{C} 12$ & $\mathrm{C} 22$ & $\mathrm{C} 27$ & $-58.2(4)$ & $\mathrm{C} 27$ & $\mathrm{C} 22$ & $\mathrm{C} 23$ & $\mathrm{C} 24$ & $0.5(4)$ \\
\hline C12 & $\mathrm{C} 22$ & $\mathrm{C} 23$ & $\mathrm{C} 24$ & $-178.4(2)$ & $\mathrm{C} 22$ & $\mathrm{C} 23$ & $\mathrm{C} 24$ & $\mathrm{C} 25$ & $-0.2(4)$ \\
\hline $\mathrm{C} 23$ & $\mathrm{C} 24$ & $\mathrm{C} 25$ & C26 & $-0.3(4)$ & $\mathrm{C} 24$ & $\mathrm{C} 25$ & $\mathrm{C} 26$ & $\mathrm{C} 27$ & $0.5(4)$ \\
\hline $\mathrm{C} 25$ & $\mathrm{C} 26$ & $\mathrm{C} 27$ & $\mathrm{C} 22$ & $-0.2(4)$ & $\mathrm{C} 23$ & $\mathrm{C} 22$ & $\mathrm{C} 27$ & $\mathrm{C} 26$ & $-0.3(4)$ \\
\hline $\mathrm{C} 12$ & $\mathrm{C} 22$ & $\mathrm{C} 27$ & $\mathrm{C} 26$ & $178.6(2)$ & & & & & \\
\hline
\end{tabular}

\section{References.}

1) Kauffman, G. B.; Teter, L. A. Inorg. Syn. 1963, 7, 9.

2) Preparation: (a) Roesch, K. R.; Larock, R. C. J. Org. Chem. 2001, 66, 412-420. Data: (b) Zhai, D.; Zhai, W.; Williams, R. M. J. Am. Chem. Soc. 1988, 110, 2501-2505.

3) Murray, R. E. Synth. Commun. 1980, 10, 345-349.

4) Luo, F.-T.; Wang, M.-W.; Wang, R. T. Org. Synth. 1998, 75, 146-152.

5) Vassilikogiannakis, G.; Orfanopoulos, M. Tetrahedron Lett. 1997, 38, 4323-4326.

6) Zhang, W.; Kraft, S.; Moore, J. S. J. Am. Chem. Soc. 2004, 126, 329-335.

7) Inman, W. D.; Sanchez, K. A. J.; Chaidez, M. A.; Paulson, D. R. J. Org. Chem. 1989, 54, 4872-4881.

8) Gelman, D.; Buchwald, S. L. Agnew. Chem. Int. Ed. 2003, 42, 5993-5996.

9) Liebeskind, L. S.; South, M. S. J. Org. Chem. 1980, 45, 5426-5429.

10) Zinc was activated prior to its use and stored in a dessicator. Activation procedure: Lauritsen, A.;

Madsen, R. Org. Biomol. Chem. 2006, 4, 2898-2905.

11) Yoshida, H.; Nakajima, M.; Ogata, T. Synthesis 1981, 36-38. 